University of Tennessee Health Science Center

UTHSC Digital Commons

\title{
$5-2014$
}

\section{The Health-Related Quality of Life of Adults with Down Syndrome}

Rebecca Jermyn Graves

University of Tennessee Health Science Center

Follow this and additional works at: https://dc.uthsc.edu/dissertations

Part of the Congenital, Hereditary, and Neonatal Diseases and Abnormalities Commons, and the Nursing Commons

\section{Recommended Citation}

Graves, Rebecca Jermyn , "The Health-Related Quality of Life of Adults with Down Syndrome" (2014).

Theses and Dissertations (ETD). Paper 99. http://dx.doi.org/10.21007/etd.cghs.2014.0115.

This Dissertation is brought to you for free and open access by the College of Graduate Health Sciences at UTHSC Digital Commons. It has been accepted for inclusion in Theses and Dissertations (ETD) by an authorized administrator of UTHSC Digital Commons. For more information, please contact jwelch30@uthsc.edu. 


\title{
The Health-Related Quality of Life of Adults with Down Syndrome
}

\begin{abstract}
Purpose. The purpose of this study was to examine the health-related quality of life (HRQOL) and factors associated with the HRQOL of adults with Down syndrome (DS) as reported by adults with DS and their caregivers. The study was designed to compare measures of HRQOL, health problems, health risks, and health-care utilization of adults with DS based on self-report with those from caregiver-report and to explore associations between HRQOL and health problems, health risks, health-care utilization, adaptive behavior, and demographic characteristics. Background. The life expectancy of individuals with DS has lengthened from approximately 30 years around 1960 to over 50 years currently. As adults with DS are living longer, measures such as HRQOL can help health-care providers understand the impact that health has on the quality of life of adults with DS and adapt interactions and interventions appropriately based on these findings. Currently, no studies have been identified that measure the HRQOL of adults with DS with an internationally-recognized standardized instrument. Additionally, there is controversy about assessing subjective concepts, such as HRQOL through the use of a proxyrespondent, (e.g., a parent, sibling, or unrelated caregiver). Because HRQOL has not been assessed previously in adults with DS, contributing factors to HRQOL have not been explored. Methods. Applying the Life Course Perspective (LCP) as a theoretical framework, a mixedmethods design was employed in this descriptive, correlational, cross-sectional study. Established surveys, investigator-developed questionnaires, and a semi-structured interview were administered during face-to-face meetings with 60 adults with DS and their caregivers in Alabama, Florida, Mississippi, and Tennessee. Findings and Conclusions. Self-reported HRQOL of adults with DS is above average, as is caregiver-reported HRQOL of adults with DS. There were no significant associations identified between the scores of physical HRQOL of adults with DS as reported by adults with DS and by their caregivers. There were also no significant associations identified between the scores of mental HRQOL of adults with DS as reported by adults with DS and by their caregivers. Not all variables of health problems, health risks, and health-care utilization of adults with DS obtained via self-report were associated with those obtained via caregiver-report, and some significant differences were identified. Problems with teeth or gums and asthma per caregiver-report and race of adults with DS were found to predict $28.5 \%$ of the variance of self-reported physical HRQOL of adults with DS; other health problems and feeling sad or blue per self-report and number of times going to the bathroom at night and allergies per caregiver-report were found to predict $40 \%$ of the variance of self-reported mental HRQOL of adults with DS; resistance to health care and trouble walking per self-report, trouble walking and weight problems per caregiver-report, and state of residence were found to predict $54 \%$ of the variance of caregiverreported physical HRQOL of adults with DS; and problems with appetite per caregiver and living arrangements of adults with DS were found to predict $39.1 \%$ of the variance of caregiverreported mental HRQOL of adults with DS. Although adaptive behavior scores predicted one or more component of HRQOL of adults with DS in preliminary regression models, they were not predictors in the final model. Health risks did not predict either component of HRQOL. Finally, the LCP is a valid theoretical framework for investigating HRQOL of adults with DS, as expected theoretical relationships between health problems and HRQOL were supported.
\end{abstract}

Document Type

Dissertation

Degree Name

Doctor of Philosophy (PhD)

Program

Nursing Science 


\section{Research Advisor}

J. Carolyn Graff, Ph.D.

\section{Keywords}

Adaptive behavior, Adults with Down syndrome, Health-related quality of life, Life course perspective

\section{Subject Categories}

Congenital, Hereditary, and Neonatal Diseases and Abnormalities | Diseases | Medicine and Health Sciences | Nursing 
The Health-Related Quality of Life of Adults with Down Syndrome

\author{
A Dissertation \\ Presented for \\ The Graduate Studies Council \\ The University of Tennessee \\ Health Science Center \\ In Partial Fulfillment \\ Of the Requirements for the Degree \\ Doctor of Philosophy \\ From The University of Tennessee
}

By

Rebecca Jermyn Graves

May 2014 
Copyright (C) 2014 by Rebecca Jermyn Graves. All rights reserved. 


\section{DEDICATION}

This dissertation is dedicated to all those who supported me in this challenging, yet rewarding journey, especially my husband, Brian, in deep gratitude for all he has done to help and encourage me and to make this opportunity possible and somewhat reasonable for me; my parents, Thomas and Linda Jermyn, in thanksgiving for their constant support and encouragement through this process as well as all previous endeavors leading up to it; my sister, Debbie Jermyn Biggs and her family, Huntley, Sydney, and Thomas, in appreciation for their never-ending support and cheering; and my dear friend, Pamela Cotton Field and her family, especially Elijah Zade, who inspired this research and motivated me on days when it was difficult to keep going. I also dedicate this research to the amazing people that I have gotten to know as a result of it; I thank them for welcoming me into their lives and their homes. I hope that the work I have done can benefit each person in at least some small way. 


\section{ACKNOWLEDGEMENTS}

I would like express my gratitude to Dr. Carolyn Graff, my dissertation committee chair. Dr. Graff has taught me formally, but also through example by her words, actions, attitude, and disposition. I have benefitted immensely from her guidance, patience, and support, and I consider myself extremely fortunate to have worked so closely with her during this program.

I would also like to thank the other members of my committee: Dr. Anna Esbensen who provided me with guidance, resources, and encouragement that made me believe that my work will be a positive contribution to the body of knowledge of adults with Down syndrome; Dr. Donna Hathaway who shared her expertise in quality of life research as well as nursing research and who introduced new ideas and perspectives during every meeting; Dr. Jim Wan who patiently walked me through the intimidating and unfamiliar process of statistical analysis with kindness; and Dr. Mona Wicks who contributed her expertise in caregiver research and nursing research and who helped guide me with her gentle demeanor, thoughtful words, and invaluable ideas.

I want to express my appreciation to the Down Syndrome Society of Mobile County in Mobile, Alabama for offering me their first research grant to support my work which enabled me to provide incentive gift cards for the participants of my study and to purchase a license to use a copyrighted instrument. I would also like to thank the Beta Theta-at-large chapter of Sigma Theta Tau International Honor Society of Nursing for awarding me a grant to cover the rest of the expenses, especially those for travel and administrative costs. 


\begin{abstract}
Purpose. The purpose of this study was to examine the health-related quality of life (HRQOL) and factors associated with the HRQOL of adults with Down syndrome (DS) as reported by adults with DS and their caregivers. The study was designed to compare measures of HRQOL, health problems, health risks, and health-care utilization of adults with DS based on self-report with those from caregiver-report and to explore associations between HRQOL and health problems, health risks, health-care utilization, adaptive behavior, and demographic characteristics.
\end{abstract}

Background. The life expectancy of individuals with DS has lengthened from approximately 30 years around 1960 to over 50 years currently. As adults with DS are living longer, measures such as HRQOL can help health-care providers understand the impact that health has on the quality of life of adults with DS and adapt interactions and interventions appropriately based on these findings. Currently, no studies have been identified that measure the HRQOL of adults with DS with an internationally-recognized standardized instrument. Additionally, there is controversy about assessing subjective concepts, such as HRQOL through the use of a proxy-respondent, (e.g., a parent, sibling, or unrelated caregiver). Because HRQOL has not been assessed previously in adults with DS, contributing factors to HRQOL have not been explored.

Methods. Applying the Life Course Perspective (LCP) as a theoretical framework, a mixed-methods design was employed in this descriptive, correlational, cross-sectional study. Established surveys, investigator-developed questionnaires, and a semi-structured interview were administered during face-to-face meetings with 60 adults with DS and their caregivers in Alabama, Florida, Mississippi, and Tennessee.

Findings and Conclusions. Self-reported HRQOL of adults with DS is above average, as is caregiver-reported HRQOL of adults with DS. There were no significant associations identified between the scores of physical HRQOL of adults with DS as reported by adults with DS and by their caregivers. There were also no significant associations identified between the scores of mental HRQOL of adults with DS as reported by adults with DS and by their caregivers. Not all variables of health problems, health risks, and health-care utilization of adults with DS obtained via self-report were associated with those obtained via caregiver-report, and some significant differences were identified. Problems with teeth or gums and asthma per caregiver-report and race of adults with DS were found to predict $28.5 \%$ of the variance of self-reported physical HRQOL of adults with DS; other health problems and feeling sad or blue per self-report and number of times going to the bathroom at night and allergies per caregiver-report were found to predict $40 \%$ of the variance of self-reported mental HRQOL of adults with DS; resistance to health care and trouble walking per self-report, trouble walking and weight problems per caregiver-report, and state of residence were found to predict $54 \%$ of the variance of caregiver-reported physical HRQOL of adults with DS; and problems with appetite per caregiver and living arrangements of adults with DS were found to predict $39.1 \%$ of the variance of caregiver-reported mental HRQOL of adults with DS. 
Although adaptive behavior scores predicted one or more component of HRQOL of adults with DS in preliminary regression models, they were not predictors in the final model. Health risks did not predict either component of HRQOL. Finally, the LCP is a valid theoretical framework for investigating HRQOL of adults with DS, as expected theoretical relationships between health problems and HRQOL were supported. 


\section{TABLE OF CONTENTS}

CHAPTER 1. INTRODUCTION ......................................................................................1

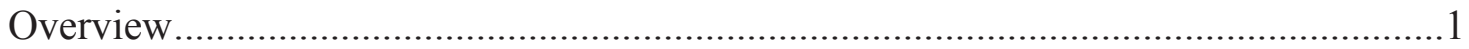

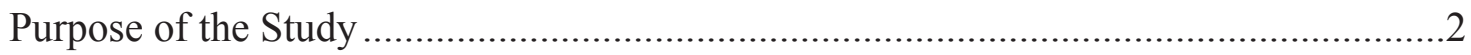

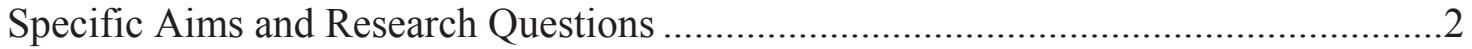

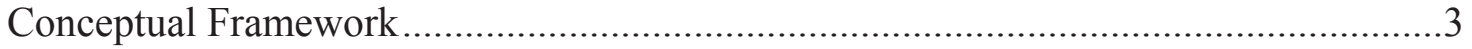

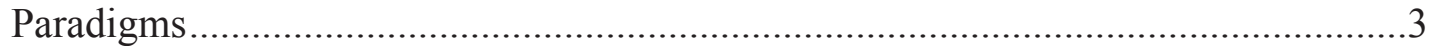

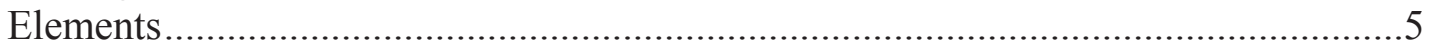

Theoretical application.......................................................................................

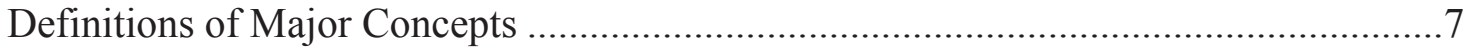

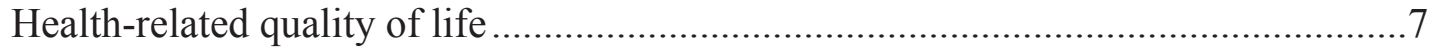

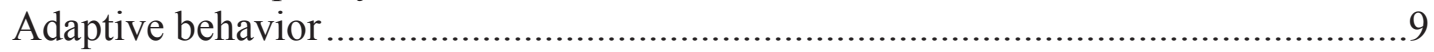

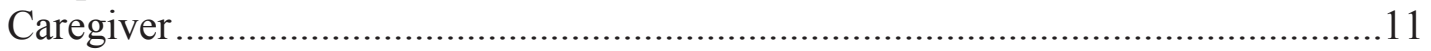

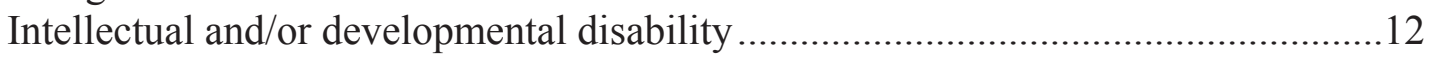

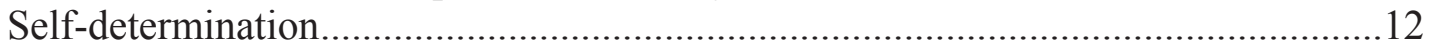

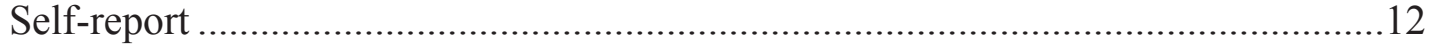

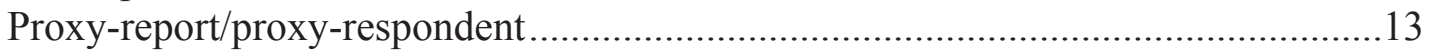

Participant dyad ........................................................................................ 13

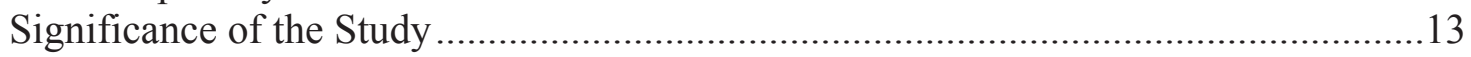

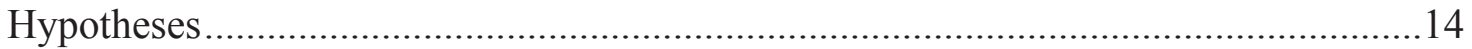

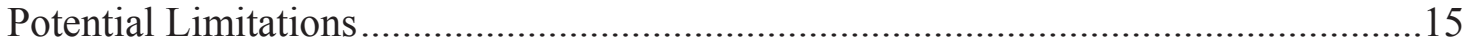

CHAPTER 2. REVIEW OF LITERATURE ...........................................................16

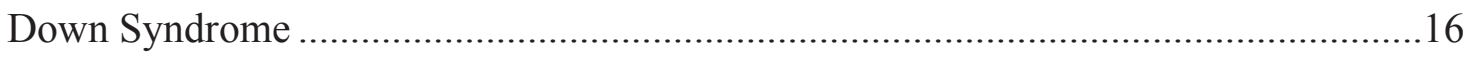

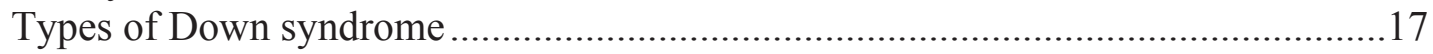

Health problems of individuals with Down syndrome .......................................18

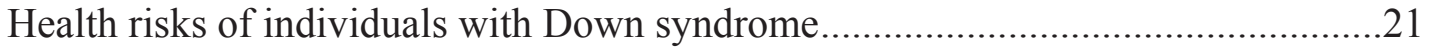

Health-care utilization by individuals with Down syndrome .................................22

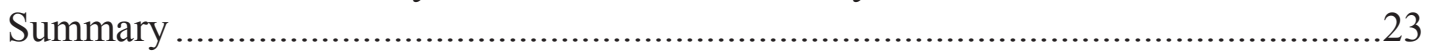

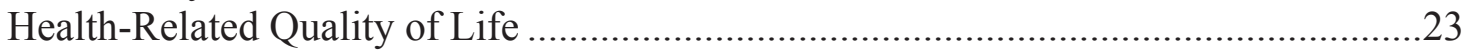

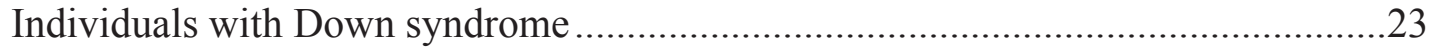

Individuals with an intellectual and/or developmental disability ...........................24

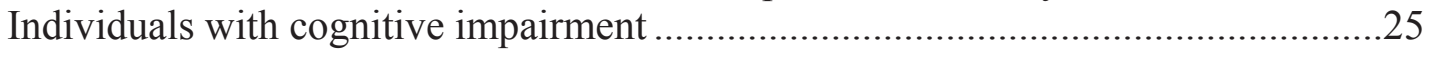

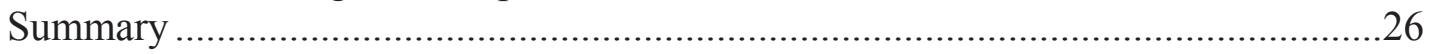

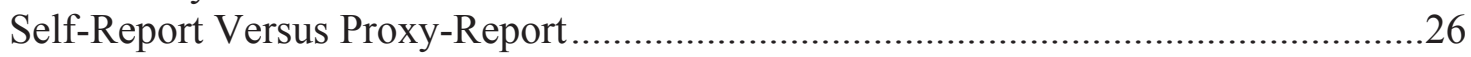

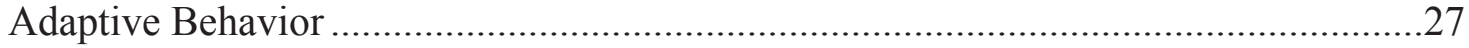

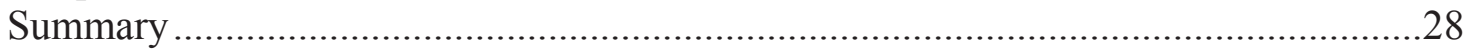

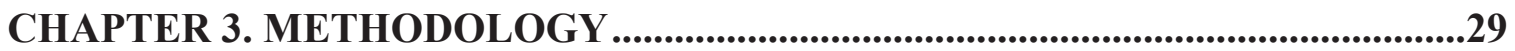

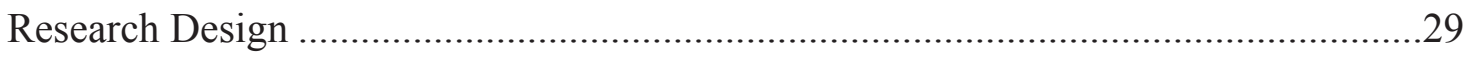

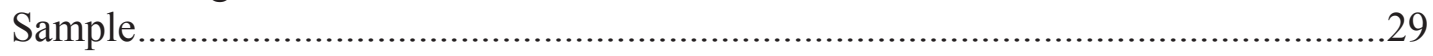

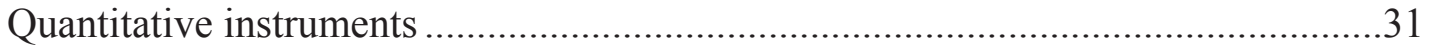

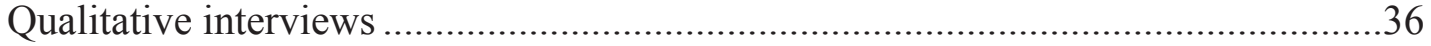

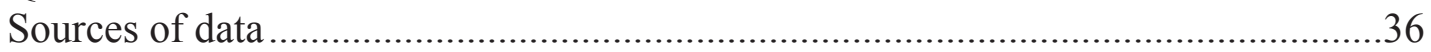




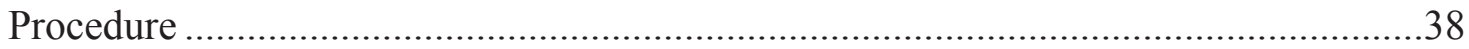

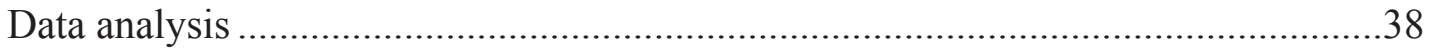

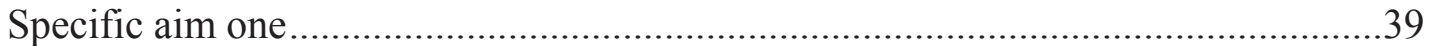

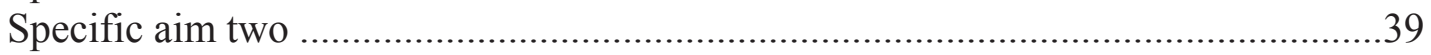

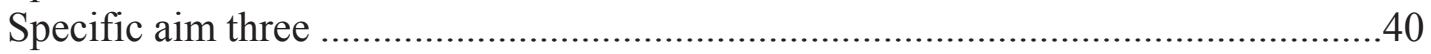

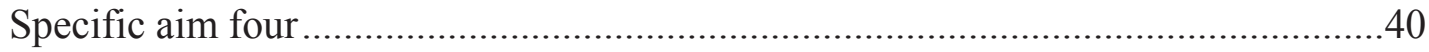

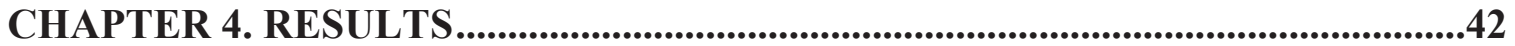

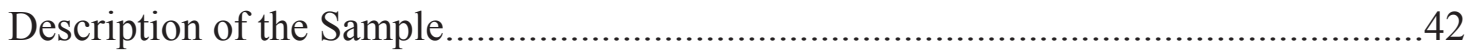

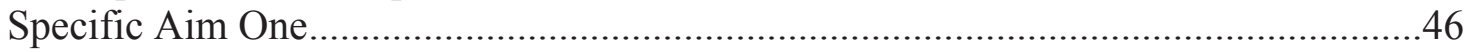

Research question 1a findings .........................................................................47

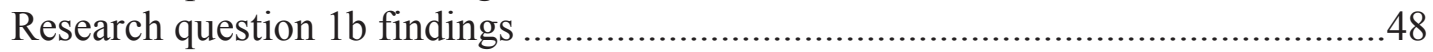

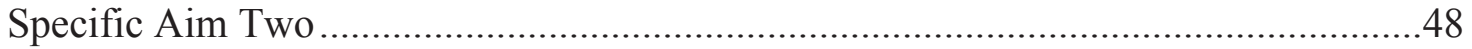

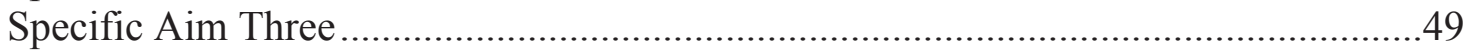

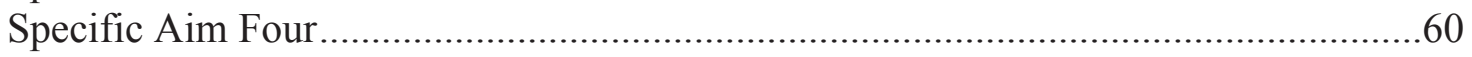

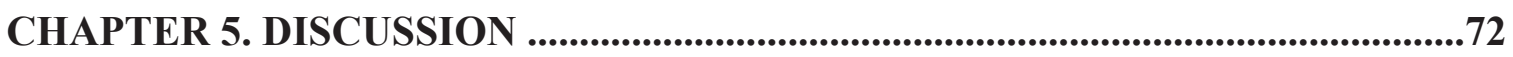

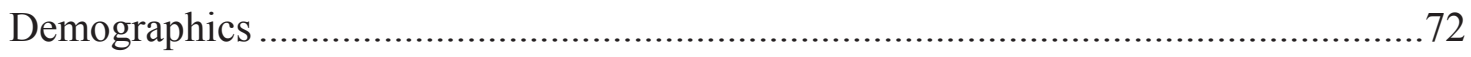

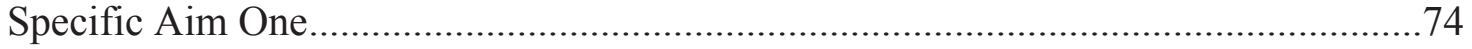

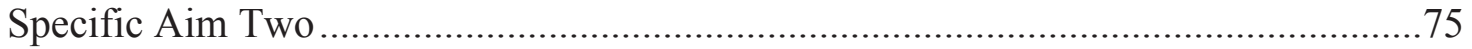

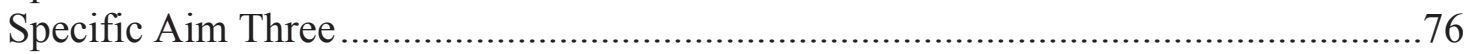

Health problems of adults with Down syndrome ……….........................................76

Health risks of adults with Down syndrome ............................................................83

Health-care utilization by adults with Down syndrome ………………....................84

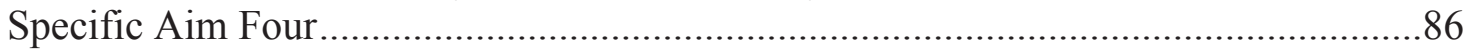

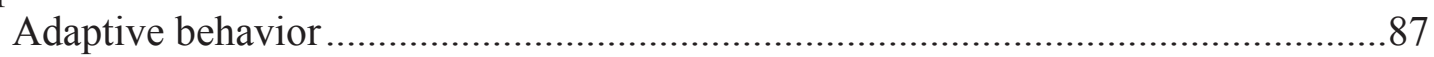

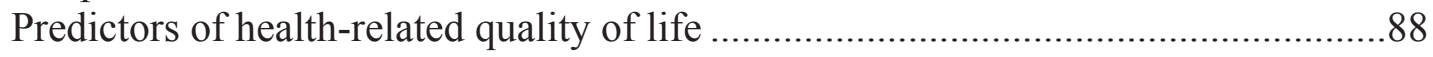

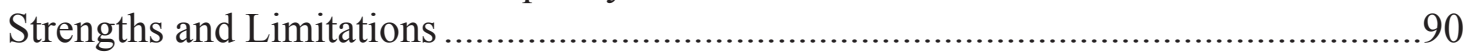

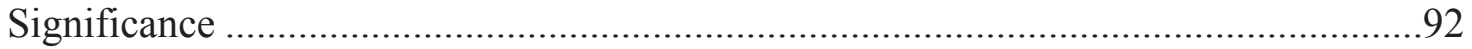

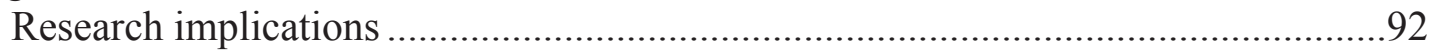

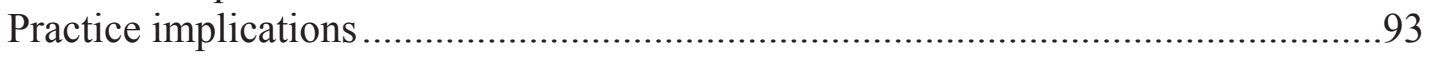

Theoretical implications...............................................................................94

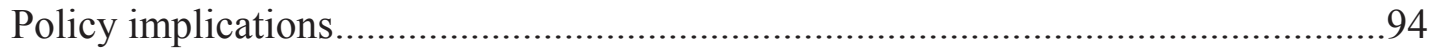

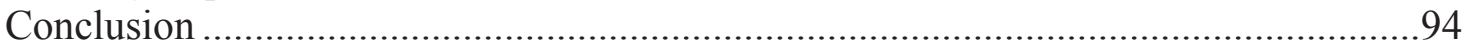

LIST OF REFERENCES .............................................................................................96

APPENDIX A. CONSENT FORM FOR PARTICIPANTS WITH DOWN

SYNDROME ..............................................................................................................111

APPENDIX B. CONSENT FORM FOR CAREGIVER PARTICIPANTS..............118

APPENDIX C. RECRUITMENT FLYER .......................................................................123

APPENDIX D. DEMOGRAPHIC FORM …................................................................124

APPENDIX E. HEALTH QUESTIONNAIRE ................................................................131 
VITA........................................................................................................................142 


\section{LIST OF TABLES}

Table 3-1. Sources of data from adults with Down syndrome and their caregivers ......37

Table 4-1. Demographic characteristics of adults with Down syndrome and their

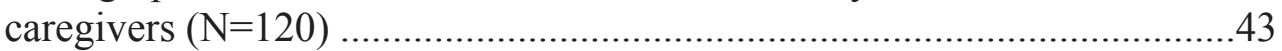

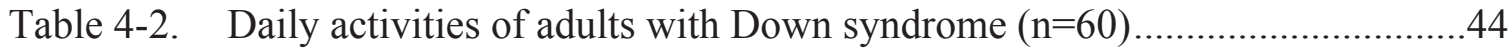

Table 4-3. Living arrangements of adults with Down syndrome and their relationships with caregivers $(n=60)$

Table 4-4. Investigator's confidence rating of Short Form-12v2 responses by adults with Down syndrome $(\mathrm{n}=60)$.

Table 4-5. Health-related quality of life scores of adults with Down syndrome obtained via self-report $(n=60)$ and caregiver-report $(n=60)$

Table 4-6. Health-related quality of life scores of adults with Down syndrome with interviewer's confidence levels 7-12 obtained via self-report $(n=44)$ and caregiver-report $(n=44)$

Table 4-7. Health problems of adults with Down syndrome obtained via self-report $(\mathrm{n}=60)$ and caregiver-report $(\mathrm{n}=60)$

Table 4-8. Health risks of adults with Down syndrome obtained via self-report $(\mathrm{n}=60)$ and caregiver-report $(\mathrm{n}=60)$

Table 4-9. Health-care utilization of adults with Down syndrome obtained via selfreport $(n=60)$ and caregiver-report $(n=60) \ldots$

Table 4-10. Associations between health-care utilization of adults with Down syndrome obtained via self-report $(n=60)$ and caregiver-report $(n=60)$

Table 4-11. Adaptive behavior scores for adults with Down syndrome

Table 4-12. Correlation of the health-related quality of life of adults with Down syndrome via self- and caregiver-report to demographic characteristics of adults with Down syndrome and their caregivers.

Table 4-13. Correlation of the health-related quality of life of adults with Down syndrome via self- and caregiver-report to health problems, health risks, and health-care utilization per self-report

Table 4-14. Correlation of the health-related quality of life of adults with Down syndrome via self- and caregiver-report to health problems, health risks, and health-care utilization per caregiver-report 
Table 4-15. Correlation of the health-related quality of life of adults with Down syndrome via self- and caregiver-report to adaptive behavior per caregiver-report

Table 4-16. Regression models with self-reported physical component summary score as dependent variable.

Table 4-17. Regression models with self-reported mental component summary score as dependent variable

Table 4-18. Regression models with caregiver-reported physical component summary score as dependent variable

Table 4-19. Regression models with caregiver-reported mental component summary

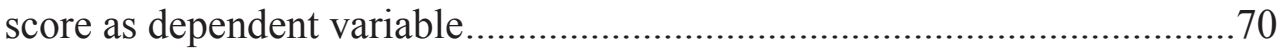

Table 4-20. Regression models for self-reported health-related quality of life . .71

Table 4-21. Regression models for caregiver-reported health-related quality of life 


\section{LIST OF FIGURES}

Figure 1-1. Model of health-related quality of life as a component of Life Course

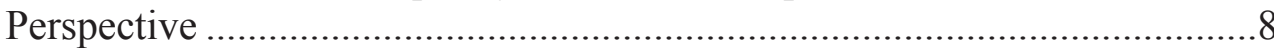




\section{LIST OF ABBREVIATIONS}

\begin{tabular}{|c|c|}
\hline $\mathrm{AD}$ & Alzheimer's disease \\
\hline $\mathrm{CP}$ & Cerebral palsy \\
\hline DS & Down syndrome \\
\hline GERD & Gastroesophageal reflux disease \\
\hline GI & Gastrointestinal \\
\hline HRQOL & Health-related quality of life \\
\hline IDD & Intellectual and/or developmental disability \\
\hline LCP & Life Course Perspective \\
\hline $\mathrm{MCS}$ & Mental component summary score \\
\hline $\mathrm{OA}$ & Osteoarthritis \\
\hline OSA & Obstructive sleep apnea \\
\hline PCS & Physical component summary score \\
\hline QOL & Quality of life \\
\hline SF-12 & Medical Outcomes Study Short Form-12 \\
\hline $\mathrm{SF}-12 \mathrm{v} 2$ & QualityMetric Short Form-12v2 Health Survey \\
\hline SPSS & $\begin{array}{l}\text { International Business Machine Corporation Statistical Package for } \\
\text { Social Sciences }\end{array}$ \\
\hline UTHSC IRB & $\begin{array}{l}\text { University of Tennessee Health Science Center Institutional } \\
\text { Review Board }\end{array}$ \\
\hline VABS & Vineland Adaptive Behavior Scales \\
\hline Vineland-II & Vineland Adaptive Behavior Scales, Second Edition \\
\hline
\end{tabular}




\section{CHAPTER 1. INTRODUCTION}

\section{Overview}

Artifacts have provided evidence of individuals with Down syndrome (DS) from as long as two thousand years ago. In the mid- to late-19th century, Dr. John Langdon Down authored a manuscript describing the phenotypical appearance of children with the condition which he called, at the time, mongolism. Later, the condition was named for this physician (Jones, 1997; Megarbane et al., 2009). Close to a century later in 1959, it was determined that DS occurred due to complete or partial trisomy on the $21^{\text {st }}$ chromosome. At that time, the life expectancy of individuals with DS was approximately 30 years (Megarbane et al., 2009).

Over the next 50 years, life expectancy of individuals with DS increased to 50 years and older, especially in industrialized nations (Bittles \& Glasson, 2004; Leonard, Msall, Bower, Tremont, \& Leonard, 2002; Megarbane et al., 2009; Smith, 2001). Because of this lengthening life-span, research on the health of adults with DS is necessary to appropriately provide care and services (Smith, 2001).

Health-related quality of life (HRQOL) is a concept that provides insight into an individual's perceived health and is used in research and practice (Guyatt, Feeny, \& Patrick, 1993). It evolved from the concept of quality of life (QOL), which is described as a state of well-being or life satisfaction that encompasses physical and mental health, relationships, achievements, beliefs, and environmental associations understood from the perspective of the individual in the context of his/her culture, principles, potential, and circumstance (World Health Organization, 1997). Health-related quality of life more precisely focuses on domains of physical and mental health; physical, emotional, social, and role function; and the individual's perception of ability and health. It has become a useful measure for assessment and evaluation of the progression of chronic disease (Draper \& Thompson, 2001; Guyatt et al., 1993); the outcome of medical and surgical interventions (Draper \& Thompson, 2001; Guyatt et al., 1993); and the economic impact of health problems and treatments on individuals, their families, and the general population (Brown, MacAdam-Crisp, Wang, \& Iarocci, 2006; Jonsson et al., 2006; Kerner, Patterson, Grant, \& Kaplan, 1998). Administratively, HRQOL provides data for financial allocation, quality control, reimbursement, and policy development (Guyatt et al., 1993).

Because HRQOL is an informative measure of the perceived effects that health and function have on an individual, its use in nursing and medical research has increased. The measurement of HRQOL to identify an individual's perception of health in the presence of a chronic condition or disability, such as DS, will be beneficial to future research and health care by providing a general understanding of how adults with DS think that their health affects their well-being. It is also valuable to establish a baseline by which to compare future assessments of HRQOL as the increased research activity results in improved interventions and health care of adults with DS. 
The literature on the HRQOL of individuals with DS is sparse (Bertoli et al., 2011; van Gameren-Oosterom et al., 2011; Verstegen et al., 2013). The single study found involving adults with DS does not specify that the HRQOL was measured, but the aspects assessed are generally consistent with the variables defining HRQOL (Bertoli et al., 2011). No studies were found documenting the use or validation of standardized, internationally-accepted instruments designed to measure the HRQOL of adults with DS.

\section{Purpose of the Study}

The purpose of this study was to examine the HRQOL and factors associated with the HRQOL of adults with DS as reported by adults with DS and their caregivers. The study was designed to compare measurements of HRQOL, health problems, health risks, and health-care utilization of adults with DS based on self-report with those from caregiver-report and to explore associations between HRQOL and health problems, health risks, health-care utilization, adaptive behavior, and demographic characteristics.

\section{Specific Aims and Research Questions}

The specific aims of this study were developed to understand the HRQOL of adults with DS by measuring the HRQOL of adults with DS via self-report and caregiverreport, comparing the measurements of HRQOL via self-report and caregiver-report, comparing data about the health of adults with DS via self-report and caregiver-report, and exploring the association of HRQOL to health, adaptive behavior, and demographic characteristics of adults with DS. To address these aims, the following research questions guided this study:

Research Question 1 What is the HRQOL of adults with DS as reported by:

a) adults with DS?

b) caregivers of adults with DS?

Research Question 2 What are the associations between the HRQOL of adults with DS as reported by adults with DS and their caregivers?

Research Question 3 What are the associations between health problems, health risks, and health-care utilization of adults with DS as reported by adults with DS and their caregivers?

Research Question 4 What are the factors (health problems, health risks, healthcare utilization, adaptive behavior, and demographic characteristics) that are associated with the HRQOL of adults with DS as reported by: 
a) adults with DS?

b) caregivers of adults with DS?

\section{Conceptual Framework}

Life Course Perspective (LCP) is a theoretic framework that evolved during the mid- to late-20th century to shape research by social scientists. Prior to 1960, most researchers evaluated cross-sectional data without regard to the effects of familial, cultural, ethnic, and socio-economic differences on the individuals participating in the research (Elder \& Giele, 2009).

After 1960, the use of longitudinal research increased as a robust method to explore social science research. Simultaneously, the use of two concepts, contextualization and birth-cohort, became more widespread in research (Elder \& Giele, 2009).

The first concept, contextualization, promotes the consideration of influences, such as family, socio-economic status, living arrangements, and experiences, prompting the understanding of how these influences contribute to the individual's current or future characteristics. Through the identification of these influences and their impact on the individual, variations among individuals were more clearly understood and explained (Elder \& Giele, 2009).

The second concept is birth-cohort, which clusters individuals based on the time and/or location of their births. Often a birth-cohort includes individuals born during a particular year or in a specific geographic area. Similar characteristics were often identified in individuals based on these cohort clusters (Elder \& Giele, 2009).

Building on these concepts, LCP developed as a theoretical framework for research promoting the consideration that an individual's current characteristics can be determined or influenced by experiences and events that have occurred throughout his or her life, including those occurring prior to birth (Elder \& Giele, 2009). Therefore, an individual is a result of these experiences and events. This LCP framework has been successfully utilized to examine health (Ben-Shlomo \& Kuh, 2002; Halfon \& Hochstein, 2002); therefore LCP could inform research related to HRQOL of adults with DS although no extant literature was identified examining HRQOL within the framework. However, life experiences and events could influence the HRQOL of adults with DS.

\section{Paradigms}

Life Course Perspective evolved through the identification of five paradigmatic principles that recount the interrelatedness of the concepts (Elder, Johnson, \& Crosnoe, 2003). These paradigms validate the importance of considering many aspects of an 
individual to thoroughly investigate a phenomenon, like HRQOL, in the appropriate context.

Principle of life-span development. An important principle to guide research conducted using LCP is that of life-span development, which indicates that an individual never ceases to develop and change (Elder \& Giele, 2009; Elder et al., 2003). Putney and Bengtson (2003) emphasize that this lifelong development should be considered cumulative, as all experiences from childhood development, including physical, mental, and emotional development, have some impact on experiences and future development throughout adolescence and adulthood.

Principle of agency. Agency supports the concept that an individual is an active participant in his/her life through personal choices and actions which contribute to that individual's life course (Elder \& Giele, 2009; Elder et al., 2003). Putney and Bengtson (2003) further explain agency as the influence an individual has in his/her own life "within the constraints of social structures and historical conditions" (p. 151).

Principle of time and place. Time and place recognize the historical and geographical contexts of an individual's existence, which influence experiences, opportunities, and disadvantages that all contribute to his/her life course (Elder \& Giele, 2009; Elder et al., 2003). Putney and Bengtson (2003) recommend investigating the individual in the context of the family and also in the further context of the environment.

Principle of timing. Timing signifies the age or time in an individual's life a landmark event occurs in comparison with societal norms, and it has influence on and is influenced by one's development (Elder \& Giele, 2009; Elder et al., 2003). Putney and Bengtson (2003) propose that there are social and cultural expectations regarding the timing of transitions, and this principle accentuates the value of meeting those expectations.

Principle of linked lives. The linked lives principle supports the intertwining of an individual's life with other lives, emphasizing the influence that one person has on another (Elder \& Giele, 2009; Elder et al., 2003). Putney and Bengtson (2003) point to multiple generations of a family and how the life of each person in each generation is mutually influential over the lives of others in the family. Additionally, this principle involves results of experiences and changes in relationships due to the occurrence of significant societal change (Putney \& Bengtson, 2003). 


\section{Elements}

Two elements, transitions and trajectories, were identified by Giele and Elder (1998) which impact one's life course. Transitions are life changes, or a change from one state to another, such as marriage, death of a loved one, or loss of employment. Typically, transitions, which can be positive or negative, promote stress and often result in redirection of an individual's life course (Black, Holditch-Davis, \& Miles, 2009; Elder \& Giele, 2009; Giele \& Elder, 1998; Seltzer, Krauss, Hong, \& Orsmond, 2001).

Trajectories are conceptual life paths that are influenced by experiences, especially transitions. Negative transitions, such as the loss of a parent or the move from independent living arrangements into a long-term-care facility, are thought to depress the trajectory, or deflect it in a negative direction. Positive transitions, such as a marriage or a promotion, typically generate an upward deflection. An individual's life trajectory generally has numerous positive and negative fluctuations (Giele \& Elder, 1998; Palmer, 2010).

\section{Theoretical application}

The paradigms discussed previously are noteworthy in relation to adults with DS and to their HRQOL as examined in this study. The principle of life-span development is important because adults with DS continue to learn and change throughout adulthood (Dressler, Perelli, Feucht, \& Bargagna, 2010; Hewitt, Hinkle, \& Miccio, 2005). The principle of life-span development is applicable to the HRQOL of adults with DS through the changing of attitudes and understanding of the effects health problems, health risks, health-care utilization, and adaptive behavior have on the transitions, and thus on the trajectories of adults with DS. Additionally, this study will look at health problems that may have affected an adult with DS throughout his/her life, such as congenital cardiovascular anomalies, and will determine if these health problems during childhood affect HRQOL of the individuals with DS during adulthood.

The principle of agency is essential in application to adults with DS because it promotes the concept of self-determination, which has been shown to increase the QOL in individuals with intellectual and/or developmental disability (IDD; Wehmeyer \& Schwartz, 1998). Adults with DS who have agency in their lives can exert independence in many areas, including decisions about wellness activities, work choices, and living arrangements (Seltzer et al., 2001; Seltzer et al., 2009). Each of these areas has the potential to influence health problems as well as health risks. Agency is represented in this study by collecting data from the adults with DS directly in addition to collecting data from the caregivers. This differs from previous research which often neglects selfdetermination in adults with DS or other IDDs by regarding data obtained via proxyreport with the same validity as that obtained via self-report (Bertoli et al., 2011; van Gameren-Oosterom et al., 2011; Verstegen et al., 2013). 
The principle of time and place for individuals with DS is noteworthy because of the increase in services since 1975 when Public Law 94-142, now titled the Individuals with Disabilities Education Act of 1975 mandated the right to free public education for children with disabilities. Strikingly positive results have been noted in many areas of the lives of the recipients of these services, including education and employment (U.S. Department of Education, 2010). Additionally, birth-cohorts of adults with DS in their late 20 s and early 30 s had the advantage of pre- or neo-natal diagnosis of structural anomalies, such as cardiac or gastrointestinal malformations and were able to receive medical and surgical interventions early in life, improving their health outcomes, life experiences, and development. However, this technology was not available to diagnose and treat older birth-cohorts of adults with DS (Bittles \& Glasson, 2004).

Geographically, accessibility to services and DS-specialized health care may not be possible in less-populated areas of the country (Birenbaum \& Cohen, 2006). The principle of time and place is an important factor in this study because data were collected from adults with DS of different ages and from different geographical areas in the Southeastern U.S. The data collected in this study have the potential to identify differences in birth-cohorts, such as superior HRQOL of younger adults with DS versus older, and/or geographical areas such as superior HRQOL of individuals who live in different states.

Adults with DS commonly have delayed timing for pertinent transitions such as marriage, career, and childbirth, but the potential still exists for these transitions (Seltzer et al., 2001; Seltzer et al., 2009). Conversely, adults with DS may experience earlier timing with health problems that are often seen primarily in the elderly, such as Alzheimer-type dementia, menopause, osteoporosis, and thyroid disease, because these conditions occur earlier in individuals with DS than in the typical population (Bosch, 2003; Cohen, 1999; Esbensen, Seltzer, \& Greenberg, 2007; McCarron, Gill, McCallion, $\&$ Begley, 2005). In addition to the association of the timing of health problems with the HRQOL of adults with DS, this principle is also investigated in this study through the association between demographic factors such as the education, employment, and marital status of adults with DS with the HRQOL of adults with DS.

Finally, the principle of linked lives is essential to adults with DS because family relationships tend to be critically important, often due to the need for some degree of lifelong care and support (Grant, Nolan, \& Keady, 2003; Heller, Caldwell, \& Factor, 2007; Seltzer et al., 2001; Seltzer et al., 2009; Stebbins, 2001). The extent of health problems has the potential to disrupt social embeddedness if an adult with DS must relocate to a long-term-care facility, which has been found to dramatically influence the life trajectories of adults with DS and living parents (Seltzer et al., 2001). The principle of linked lives is evaluated in the current study through the exploration of the relationship of each adult with DS to his/her caregiver.

The concepts of LCP will not be directly investigated in this study. Rather, the perspective will provide a lens through which to examine the impact that HRQOL has on the trajectories of adults with DS. 
The model (Figure 1-1) demonstrates the multi-dimensional nature and fluidity of an individual's trajectory in the LCP. Health-related quality of life is but one intertwined filament that make up and influence an individual's LCP trajectory. Because no previous studies were identified that utilized the LCP framework to understand concepts relevant to adults with DS, the other filaments represent hypothetical concepts that could potentially be examined for this population, including recreation, spirituality, occupation, societal roles, and conflict-management. In the current study, health problems, health risks, health-care utilization, and levels of adaptive behavior can contribute to positive and negative transitions, which can factor into changes in the trajectory of an adult with DS. Because this study has a cross-sectional design, the blunted or cut end demonstrates the time point in that individual's life when the study was conducted. In the model, HRQOL consists of interactions between health problems, health risks, health-care utilization, and adaptive behavior, as this study hypothesizes that these characteristics are associated with the HRQOL of adults with DS.

\section{Definitions of Major Concepts}

Extant literature provided definitions for the major concepts, or variables, investigated in this study. Following are the definitions of these concepts applied throughout this study.

\section{Health-related quality of life}

As discussed previously, HRQOL is a measure of how an individual perceived health affected his/her life or well-being. Health-related quality of life will be operationally defined in this study by scores on the QualityMetric Short Form-12v2 Health Survey (SF-12v2).

Health. The World Health Organization (2003) defines health as comprehensive well-being in the physical, mental, and social realms of an individual's life. The definition goes on to clarify that, although this includes lack of illness, health encompasses more than just physical wellness. Health is considered to be on a continuum. Life Course Perspective has provided the conceptual framework for epidemiological studies on chronic disease, because, as researchers acknowledged, health and illness are influenced by extensive numbers of variables occurring throughout an individual's life (Ben-Shlomo \& Kuh, 2002). These variables were recognized to be both biological and psychosocial, and they contributed to both physical and mental health. When evaluating health through the framework of LCP, the fluctuations of health across the continuum are consistent with variations in the direction of an individual's trajectory, especially if the fluctuation of health is substantial enough to instigate a transition. Health-problems, health risks, and health-care utilization will be assessed during the health interview of adults with DS to provide self-report and the Health Questionnaire to provide caregiver-report. 


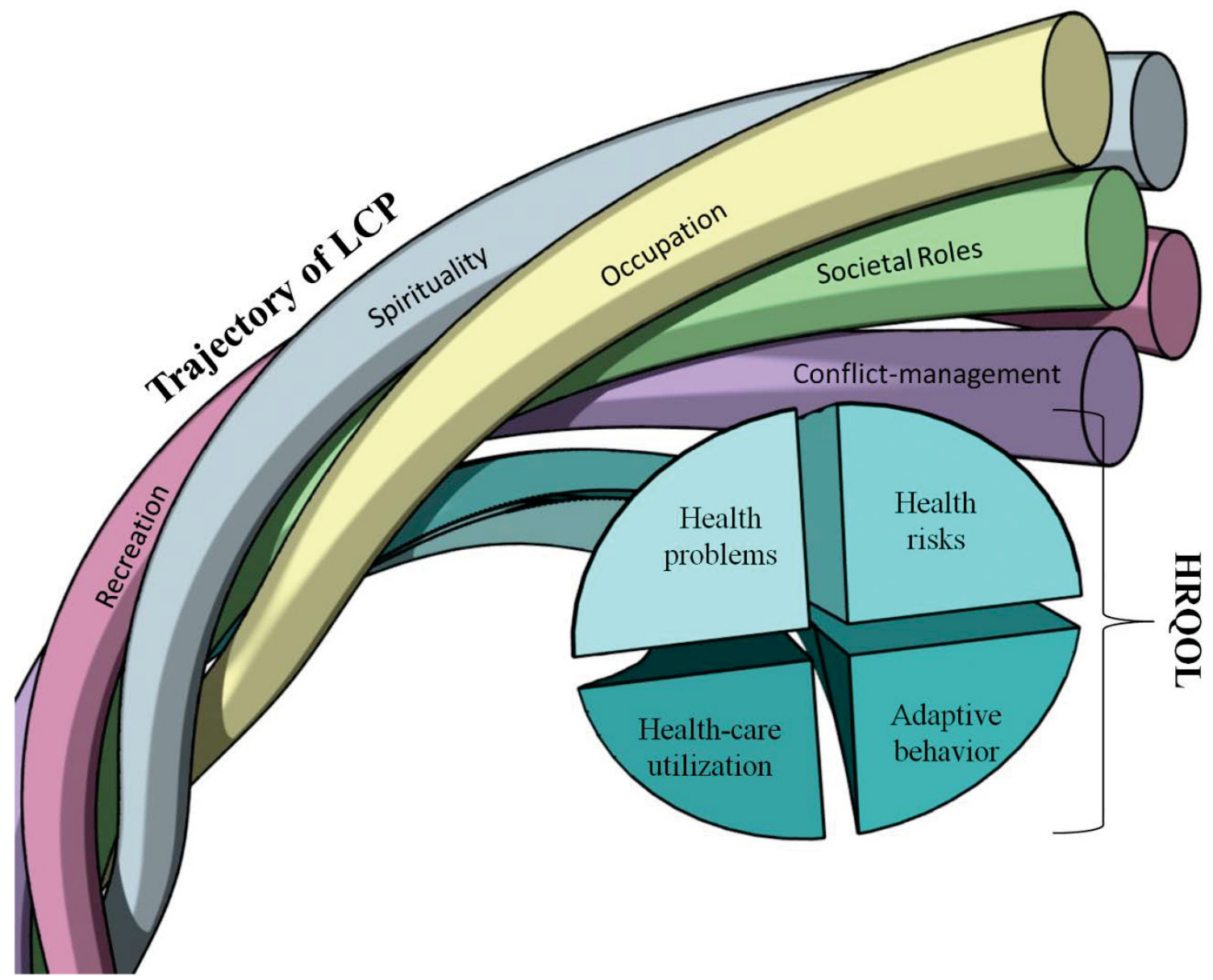

Figure 1-1. Model of health-related quality of life as a component of Life Course Perspective 
Physical health. Physical health is defined as physical well-being, with the inclusion of regular physical activity, adequate rest, hygiene, clean air and water, and nutritional items that contribute to appropriate bodily functions and energy (Schalock et al., 2010). As with the overall definition of health, physical health is also a continuum. The perception of the impact that physical health has on the HRQOL of adults with DS will be operationally defined by the physical component summary score (PCS) of the SF$12 \mathrm{v} 2$.

Mental health. Mental health is defined as possession and expression of the emotional stability and adaptability necessary to handle routine and unusual situations that occur during daily life (McGuire \& Chicoine, 2006). Mental health exists on a continuum as well. The perception of the impact that mental health has on the HRQOL of adults with DS will be operationally defined by the mental component summary score (MCS) of the SF-12v2.

\section{Adaptive behavior}

Adaptive behavior includes activities that enable a person to interact with others and to understand and function within his/her surroundings (Schalock et al., 2010; Woolf, Woolf, \& Oakland, 2010). The adaptive behaviors evaluated in this study are those involving communication, daily living skills, and socialization (Sparrow, Cicchetti, \& Balla, 2005). Adaptive behavior is associated with the LCP principles of life-span development, agency, timing, and linked lives. An individual with DS generally has impaired adaptive behavior (Schalock et al., 2010), but he/she can continue to learn skills and adaptive behaviors, or develop, throughout the life-span (Grant et al., 2003; Heller et al., 2007; Seltzer et al., 2001; Seltzer et al., 2009; Stebbins, 2001). This can result in delayed timing of developmental landmarks (Schalock et al., 2010), and, frequently, creates a need for help from a trusted family member or companion (Esbensen, Bishop, Seltzer, Greenberg, \& Taylor, 2010; Hodapp \& Urbano, 2007; McGuire \& Chicoine, 2006) demonstrating linked lives. The principle of agency is applicable to adaptive behavior because one facet of adaptive behavior included an individual's independence (Sparrow et al., 2005), which is an important component of agency. Additionally, an individual can improve his/her agency by increasing mastery of adaptive behavior skills that contribute to independence. Overall adaptive behavior will be measured by the adaptive behavior composite standard score on the Vineland Adaptive Behavior Scales, Second Edition (Vineland-II).

Communication. Anderson, Anderson, and Glanze (1998) define communication as the means through which information is conveyed between beings, primarily from one person to others. Overall communication adaptive behavior will be measured by the communication domain standard score on the Vineland-II. The communication dynamics investigated in this study are receptive, expressive, and written (Sparrow et al., 2005). 
Receptive communication. Receptive communication involves an individual's ability to pay attention, listen, and comprehend information or a message conveyed by someone else (Sparrow et al., 2005). Adults with DS may exhibit some difficulty with receptive communication due to decreased cognitive ability, which can impair processing and understanding (McGuire \& Chicoine, 2006). Adaptive behavior involving receptive communication will be measured by the receptive communication subdomain v-scale score on the Vineland-II.

Expressive communication. Expressive communication is the ability to formulate and transmit a message to others (McGuire \& Chicoine, 2006; Sparrow et al., 2005). McGuire and Chicoine indicate that cognitive limitations and articulation difficulties can impair expressive verbal communication in adults with DS. Adaptive behavior involving expressive communication will be measured by the expressive communication subdomain v-scale score on the Vineland-II.

Written communication. The written communication evaluated in this study involves literacy, or the ability to read and write (Buckley, 2001; Sparrow et al., 2005). Strong educational programs can help individuals with DS attain the abilities to read and write at a useful level by adulthood (Buckley, 2001). Adaptive behavior involving written communication will be measured by the written communication subdomain vscale score on the Vineland-II.

Daily living skills. Daily living skills, also known as activities of daily living, are actions performed throughout a typical day for a person able to care for himself/herself (Potter, 2009). Overall daily living skills adaptive behavior will be measured by the daily living skills domain standard score on the Vineland-II. The daily living skills assessed in this study are personal, domestic, and community (Sparrow et al., 2005).

Personal daily living skills. Personal daily living skills, according to Sparrow et al. (2005), are activities required to care for one's self. These skills include eating, drinking, toileting, dressing, bathing, grooming, and meeting one's personal medical needs (e.g., blowing own nose with a tissue or following prescribed medical regimens; Sparrow et al., 2005). Adaptive behavior involving personal daily living skills will be measured by the personal daily living skills subdomain v-scale score on the Vineland-II.

Domestic daily living skills. Domestic daily living skills are activities to care for one's home environment. These include maintaining home safety, doing chores in the kitchen, and keeping house (Sparrow et al., 2005). Adaptive behavior involving domestic daily living skills will be measured by the domestic daily living skills subdomain v-scale score on the Vineland-II. 
Community daily living skills. Community daily living skills are activities necessary to live and function in the community. These include the ability to use the telephone; understand rules, rights, and safety outside the home; comprehend times and dates; perform skills needed to work at a job; use and count money accurately; order a meal in a restaurant; operate electronic equipment including a radio, television, and computer; and leave the home independently (Sparrow et al., 2005). Adaptive behavior involving community daily living skills will be measured by the community daily living skills subdomain v-scale score on the Vineland-II.

Socialization. Socialization represents behaviors that permit congenial association and interaction with others. Overall socialization adaptive behavior will be measured by the socialization domain standard score on the Vineland-II. The areas of socialization reviewed in this study are interpersonal relationships, play and leisure time, and coping (Sparrow et al., 2005).

Interpersonal relationships. Interpersonal relationships are ways that individuals intermingle with others. The behaviors inspected are responding to others, expressing and recognizing emotions, imitating others, communicating socially, being thoughtful, having friendships, and dating (Sparrow et al., 2005). Adaptive behavior involving interpersonal relationships will be measured by the interpersonal relationships subdomain v-scale score on the Vineland-II.

Play and leisure time. Play and leisure time represents how an individual behaves in situations intended to be enjoyable. This includes playing informally and with games, sharing and cooperating, going places with friends, and recognizing social cues (Sparrow et al., 2005). Adaptive behavior involving play and leisure time will be measured by the play and leisure time subdomain v-scale score on the Vineland-II.

Coping skills. Coping skills determine how an individual adapts to different situations while displaying dependability and kindness to others. The coping skills appraised are manners, apologies, responsibility, appropriate social caution, transitions (e.g., changes in activity or changes in routine), control of impulses, and keeping secrets (Sparrow et al., 2005). Adaptive behavior involving coping skills will be measured by the coping skills subdomain v-scale score on the Vineland-II.

\section{Caregiver}

While the term caregiver can have many different definitions, in this study it is defined as a family member or friend on whom an individual relies for help to meet his/her physical and emotional needs (Stebbins, 2001). The caregivers in this study are informal, unpaid caregivers who have considerable familiarity with the adults with DS 
participating in the study, often a family member or friend living with the adult with DS. Many adults with DS live with their parents or other family members (Esbensen et al., 2010; Hodapp \& Urbano, 2007; McGuire \& Chicoine, 2006), while some live independently alone, with roommates, or with significant others. Other adults with DS live in supervised/staffed homes, apartments, or larger institutions (Hodapp \& Urbano, 2007). Life Course Perspective supports the inclusion of caregivers in the current study through the principle of linked lives. As stated previously, this is an important component in the life of an individual with DS (Grant et al., 2003; Heller et al., 2007; Seltzer et al., 2001; Seltzer et al., 2009; Stebbins, 2001)

\section{Intellectual and/or developmental disability}

Resulting from the evolution of terminology over decades (Crocker, 2006b), it is challenging to find one accepted comprehensive definition of intellectual and/or developmental disability. For the purposes of this study, the definitions of intellectual disability and developmental disability have been combined. Therefore, an IDD is a lifelong cognitive impairment manifested before adulthood that causes limitations of intellectual function and adaptive behavior affecting abilities for learning, daily living skills, mobility, independence, and social interaction requiring varying levels of enduring support and assistance (Developmental Disabilities Assistance and Bill of Rights Act of 2000 [Public Law 106-402; 102 (8)]; Schalock et al., 2010). Down syndrome is an IDD; therefore the theoretical application of the LCP principles discussed previously is relevant for this study. Other examples of IDDs include cerebral palsy, fragile-X syndrome, and autism (Nehring \& Betz, 2010).

\section{Self-determination}

The definition of self-determination is autonomy, or the conviction that all individuals are decision-making participants in their lives (Schalock et al., 2010; Wehmeyer \& Bolding, 2001). This study supported the self-determination of adults with DS by obtaining subjective measures directly from adults with DS, not exclusively from caregivers and by obtaining the consent of the adults with DS for the caregivers to divulge private information about the adults with DS for the study. Self-determination is a component of the LCP principle of agency.

\section{Self-report}

When an individual answers interview or survey questions about himself/herself, it is considered self-report (Polit \& Beck, 2008). Often, individuals with DS or other IDDs are not directly asked to provide self-report for subjective questions about himself/herself because of anticipated or actual cognitive and communication limitations (Brown, 1998; Fujiura \& Behrens, 2008). In this study, adults with DS verbally completed the SF-12v2 to ascertain their HRQOL via self-report. They were also 
interviewed using questions to encourage them to discuss their health problems, health risks, and health-care utilization to better understand the subjectivity of these factors. Self-report also supports the LCP principle of agency since it respects self-determination and the understanding that an individual is able to influence his/her LCP trajectory.

\section{Proxy-report/proxy-respondent}

Much research with individuals with IDD utilizes a proxy-respondent, or an individual who knows the primary research participant well and answers subjective questions on his/her behalf, providing what is considered proxy-report (Polit \& Beck, 2008; Zimmermann \& Endermann, 2008). This has been the solution often used when researchers believed that self-report was unreliable (Brown, 1998; Fujiura \& Behrens, 2008). The caregivers in this study participated as proxy-respondents for the adults with

DS. However, the HRQOL survey and the health problems, health risks, and health-care utilization were collected from both the adult with DS and the caregiver to determine the associations between self-report and proxy-report (caregiver-report) in this sample.

Questioning a proxy-respondent is related to the LCP principle of linked lives, regarding the intertwined experiences of the adult with DS and his/her caregiver.

\section{Participant dyad}

A participant dyad in research represents a pair of people participating in a study together, often one individual with a particular characteristic of interest (e.g., a diagnosis) and his/her caregiver. This is often done to understand influences on and of the individual requiring care (Quinn, Dunbar, Clark, \& Strickland, 2010). Participant dyads, each consisting of an adult with DS and his/her caregiver, were recruited in this study to understand associations between self- and caregiver-report of HRQOL, health problems, health risks, and health-care utilization of adults with DS. The participant dyads investigated in this study is further demonstration of the LCP principle of linked lives.

\section{Significance of the Study}

The concept of HRQOL is used to guide and evaluate medical care for individuals with chronic, or lifelong, medical problems (Draper \& Thompson, 2001; Guyatt et al., 1993). As discussed previously, there is virtually no existing literature on the HRQOL of adults with DS, including literature using standardized instruments to measure HRQOL in this population. This study seeks to establish a baseline measurement and provide context of the HRQOL of adults with DS using an internationally acknowledged standardized instrument that measures HRQOL in adults. The results of this study will be useful in guiding future research on the health and HRQOL of adults with DS. It also can contribute to interventions used in health care to assess and improve the HRQOL of adults with DS. By exploring the associations of HRQOL with variables of demographic characteristics, health problems, health risks, health-care utilization, and adaptive 
behavior, researchers can pursue the examination of relevant findings to further understand the associations and to develop and investigate interventions to improve HRQOL. Additionally, the results of this study can provide information about the importance of interacting with the adult with DS as well as his/her caregiver as their perceptions about health and HRQOL may differ on key issues. Identification of associations between HRQOL with variables of demographic characteristics, health problems, health risks, health-care utilization, and adaptive behavior can provide policymakers with justification to support increased resources for and improvement of associated variables, thus, improving the HRQOL of adults with DS.

The importance of the measurement of HRQOL of adults with DS obtained via self-report is upheld by Brown (1998), who maintained that there are discrepancies with proxy-report, because others are unable to completely understand someone's insight with absolute acuity. Cummins (1997) explained the inconsistency of proxy-report as basing responses to questions about the perspective of an individual with an IDD on the perception of expectations, circumstances, and experiences of a person without an IDD. While proxy-respondents are often very familiar with the individual for whom they are responding, and objectively-obtained subjective data can potentially be an exact reflection of the participant's thoughts, the precision of personal perspective is forfeited through the use of a proxy-respondent (Stade, Stevens, Ungar, Beyene, \& Koren, 2006). This study will evaluate the associations between self-report and caregiver-report to steer future research on HRQOL and health of adults with DS. These data will be informative in the health-care arena as well, because health-care providers can gain insight on the accuracy of caregiver-report regarding symptoms and health problems and may be encouraged to give equal consideration to both caregiver-report and self-report of adults with DS.

Finally, this study will identify the underpinnings of importance the adult with DS assigns to his/her own health problems, health risks, and health-care utilization. This information will not only enrich the data obtained in the study, it can also provide an understanding upon which to base future research, health care, interventions, and education.

\section{Hypotheses}

The following hypotheses have been formulated for the current study:

1) The mean HRQOL score of adults with DS obtained via self-report will be above average, or a mean higher than 50.0 on the PCS and on the MCS of the SF-12v2. The mean PCS and MCS scores of the HRQOL of adults with DS obtained via caregiver-report will be average to above average.

2) As indicated in the first hypothesis, a difference between self-reported HRQOL of adults with DS and that reported by caregivers will be detected by the proposed study design.

3) The SF-12v2 will be applicable to measure HRQOL of adults with DS. 
4) The reports of health problems, health risks, and health-care utilization of adults with DS via self-report will be comparable to that reported by caregivers. However, the caregiver-report will likely be more detailed.

5) Associations will be identified between the HRQOL of adults with DS and their health problems, health risks, health-care utilization, adaptive behavior, and demographic characteristics. The hypothesis is that increasing health problems and health risks will be negatively associated with HRQOL. An additional hypothesis is that increasing health-care utilization, in the form of routine screenings and exams (proactive), and adaptive behavior composite scores will be positively associated with HRQOL. However, increased health care in the form of problem management (reactive) will likely be associated with decreased HRQOL.

6) The paradigms of LCP are relevant to the HRQOL of adults with DS, which can be viewed in a cross-sectional point of an individual's personal trajectory.

\section{Potential Limitations}

The anticipated limitations for this study are:

1) It will be challenging to recruit a sample size large enough to achieve statistical power for quantitative analysis, especially for multiple regression.

2) Cognitive and/or communication impairments of some adults with DS could prevent complete confidence of the validity of the data obtained from the surveys and interviews.

3) The participants in the study will be recruited through non-probability convenience sampling methods which may lend bias and prevent a more representative sample.

Participants will be recruited from larger cities in the southeastern U.S. Therefore, findings from the study may not be generalizable to individuals in rural areas of the southeastern U.S., other regions of the U.S., or globally. 


\section{CHAPTER 2. REVIEW OF LITERATURE}

The health-related quality of life (HRQOL) of adults with Down syndrome (DS) is an area that has been fundamentally overlooked in the literature. Therefore, to provide a basis for investigating this phenomenon, an understanding of the concepts and all contributing factors needs to established. The major variables of this study are the physical component summary scores and mental component summary scores of the QualityMetric Short Form-12v2 Health Survey (Ware et al., 2007) that represent the HRQOL of adults with DS. The minor variables investigated in this study are the health problems, health risks, health-care utilization, and adaptive behavior of adults with DS. The following chapter will present the major and minor variables investigated in this study, discuss DS and related concepts including physical health and mental health, and provide supporting extant research.

\section{Down Syndrome}

Down syndrome is caused by an aberration on the 21 st chromosome that occurs prior to fertilization or during gestation (Crocker, 2006c; Jones, 1997; Nehring \& Betz, 2010 ) and is the most common cause of aneuploidy, where abnormal numbers of chromosomes are found in cells (Jorde, 2010; Mai et al., 2013). The incidence of infants born with DS in the U.S. from 2006-2010 was 13.1 per 10,000 pregnancies based on the National Birth Defects Prevention Network Congenital Malformations Surveillance Report (Mai et al., 2013). Of the 40 states that contributed data to this report, 11 states reported live births only with an incidence of 12.5 per 10,000, 17 states reported live births and stillbirths with an incidence of 12.6 per 10,000, and 12 states reported all pregnancy outcomes, including reported terminations, with an incidence of 14.2 per 10,000. Based on a systematic review, Natoli, Ackerman, McDermott, and Edwards (2012) determined that $50-85 \%$ of pregnancies with a prenatal diagnosis of DS are terminated. Because the incidence of DS is generally reported in number of live births by surveillance programs, or as in this report a discrepancy of pregnancy outcomes, the instances of termination cause misinterpretation of the actual occurrence of DS or other prenatally identifiable birth defects (Ethen \& Canfield, 2002). Therefore, the natural incidence of DS would be much higher if all termination data were included.

While DS occurs across races, ethnicities, nationalities, and socio-economic groups without discrimination (Nehring \& Betz, 2010), there are a number of physical features that are seen in most individuals with DS that occur across all groups. The features include a small head, ears, and mouth; a low nasal bridge; eyes that slant upward and have epicanthal folds; and a small stature (Crocker, 2006a; Jones, 1997; Jorde, 2010; Zigman, 2013). In addition to outwardly visible characteristics of the phenotype, individuals with DS have cognitive impairments, usually categorized as mild to moderate (Jones, 1997; Nehring \& Betz, 2010), and have increased incidences of health problems, both at birth and throughout their lives, discussed below. 


\section{Types of Down syndrome}

There are three general karyotypes, or chromosomal profiles, of DS that are based upon the origin of abnormality on the 21st chromosome (Jones, 1997; Jorde, 2010; Nehring \& Betz, 2010; Zigman, 2013). These are nondisjunction, translocation, and mosaicism.

Nondisjunction. Nondisjunction is the most common source of abnormality on the 21 st chromosome that is found in DS, causing approximately $95-97 \%$ of the cases (Jorde, 2010; Nehring \& Betz, 2010). Nondisjunction is an error in the separation of genetic material during cell division in one of the gametes prior to fertilization. Individuals with DS from nondisjunction have an additional 21st chromosome (three total) in every cell in his/her body (Jorde, 2010; Nehring \& Betz, 2010), resulting in the phenotype discussed previously. Parents of an individual with DS caused by nondisjunction have a very low probability of having a second child with DS (1\% in mothers 35 years old and younger).

Translocation. Translocation occurs when two different chromosomes exchange genetic material, which usually occurs between the 21 st and 14th, both 21 st, or the 21 st and 22nd chromosomes (Jones, 1997; Nehring \& Betz, 2010). Because the long-arm of the chromosome is exchanged, translocation fundamentally creates a new $21 \mathrm{st}$ chromosome since the short arm of these chromosomes either does not play a role in gene expression on these chromosomes or is not replicated in further cell division cycles. When the individual with the translocation has offspring, half of his/her gametes carry the additional copy of the 21 st chromosome, which will actuate DS in that child (Jorde, 2010). As a result of translocation, all of the offspring's cells contain three copies of the 21 st chromosome; therefore the phenotype is the same as that of DS originating from nondisjunction (Jorde, 2010; Nehring \& Betz, 2010). Translocation is the source of approximately $3-5 \%$ of the cases of DS, and because it is heritable, the probability of having a second child with DS increases to approximately $10 \%$ if originating from the mother and 5\% if originating from the father.

Mosaicism. Mosaicism, or mosaic DS, occurs in approximately $1 \%$ of individuals with DS (Jones, 1997; Jorde, 2010; Zigman, 2013). It is also caused by nondisjunction, but occurs after fertilization of gametes. Because typical cell division has already been initiated in the zygote, two cell lines, one euploid line with 46 chromosomes and one aneuploid line with 47, continue to proliferate. Not all cells in individuals with mosaic DS contain three copies of the 21 st chromosome, which dilutes the phenotypic presentation of DS in the individual (Jones, 1997; Jorde, 2010; Nehring \& Betz, 2010). 


\section{Health problems of individuals with Down syndrome}

It has been noted that there is an increased occurrence of physical and mental deterioration among adults with DS at an earlier age and more rapidly than among adults without DS (Alexander et al., 1997; Esbensen, Seltzer, \& Krauss, 2008; Finlayson, Morrison, Jackson, Mantry, \& Cooper, 2010; Smith, 2001). Health care for adults with DS should follow the same model as that for adults without DS, but with additional attention devoted to these diagnoses that occur more frequently or earlier in life than in the typical population (Crocker, 2006a).

Alzheimer's disease/dementia. Alzheimer's disease (AD) or Alzheimer-type dementia develops approximately twenty years earlier and has an increased occurrence in adults with DS than those in the general population (Bosch, 2003; Cohen, 1999; Esbensen, Seltzer, \& Greenberg, 2007; Galley, 2005; Kerins, Petrovic, Bruder, \& Gruman, 2008; McCarron, Gill, McCallion, \& Begley, 2005; McCarron, McCallion, Fahey-McCarthy, Connaire, \& Dunn-Lane, 2010; Service \& Hahn, 2003). Diagnosis of dementia or AD may be challenging with adults with DS because, due to impaired communication abilities and pre-existing cognitive impairment, the usual symptoms are difficult to recognize. While the presentation of $\mathrm{AD}$ may differ in this population, the typical pattern of the disease occurs, including gradual cognitive decline, decreasing ability to care for one's self, and physical sequelae that lead to immobility and eventual death (McCarron et al., 2005).

Cancer. Individuals with DS are more susceptible to particular types of cancer, including acute lymphoblastic leukemias, especially acute megakaryocytic leukemia (Bosch, 2003; Esbensen et al., 2007; Kerins et al., 2008; Shimizu, Engel, \& Yamamoto, 2008; Wiseman, Alford, Tybulewicz, \& Fisher, 2009; Xavier, Ge, \& Taub, 2010), and testicular germ cell tumors (TGCT; Galley, 2005). The incidence of leukemia is generally isolated to early childhood, so it is not often diagnosed in the population of interest. There is an increase risk of TGCT in this population, but the tumors are usually receptive to treatment and cured if they are detected early (Galley, 2005).

Cardiovascular disease. Individuals with DS have a 40-50\% risk of congenital heart malformations (Bosch, 2003; Cohen, 1999; Esbensen et al., 2007; Nehring \& Betz, 2010). Fortunately, the pediatric medical community is attuned to this risk, and an echocardiogram is strongly encouraged on all newborn infants with DS, with surgical repair following if the condition is identified as severe (Bosch, 2003; Crocker, 2006a). Therefore, the primary cardiac concerns for adults with DS are worsening of existing structural defects and valvular disorders, which can lead to severe complications such as pulmonary hypertension and cor pulmonale (Bosch, 2003; Cohen, 1999; Esbensen et al., 2007; Galley, 2005; Kerins et al., 2008; McCarron et al., 2005; Service \& Hahn, 2003; Virji-Babul, Eichmann, Kisly, Down, \& Haslam, 2007). Historically, it was strongly recommended to prophylactically administer antibiotics prior to dental procedures for 
those with valvular disease to prevent systemic bacterial endocarditis (Bosch, 2003; Cohen, 1999); however, this practice of antibiotic prophylaxis for individuals without DS is currently under debate (Lockhart, Hanson, Ristic, Menezes, \& Baddour, 2013).

Depression. Adults with DS appear to have a higher risk for depression than adults without DS (Bosch, 2003; Cohen, 1999; Galley, 2005; Kerins et al., 2008; McCarron et al., 2005; Virji-Babul et al., 2007). This depression often follows negative transitional events, such as the loss of a parent or caregiver (Bosch, 2003; Galley, 2005; Kerins et al., 2008). Similar to the atypical presentation of AD, adults with DS often do not follow the traditional pattern of depression, and with both diagnoses, potential physical causes of behavioral change must first be ruled out (Bosch, 2003; Galley, 2005).

Endocrine disorders. Hypothyroidism is a common endocrine disease noted in adults with DS (Bosch, 2003; Cohen, 1999; Galley, 2005; Kerins et al., 2008; McCarron et al., 2005; Service \& Hahn, 2003; Virji-Babul et al., 2007). Making a diagnosis of hypothyroidism can be challenging because, while thyrotropin (TSH) may be elevated, thyroxine (T4) levels could be within the normal range (Bosch, 2003; Cohen, 1999; Galley, 2005). Therefore it is recommended to assess both levels to avoid misdiagnosis. There is also an increased incidence of hyperthyroidism in adults with DS, but it is much less common (Cohen, 1999). Anwar, Walker, and Frier (1998) found that Type I diabetes occurs at a rate of 1.4-10.6\% of individuals with DS in an area of Scotland, while the estimated incidence of Type 1 diabetes was $0.18-.3 \%$ in the general population of the United Kingdom. Individuals with DS do not appear to be at an increased risk of Type 2 diabetes (Esbensen, 2010). In a study of 440 children with DS, $0.9 \%$ of their parents reported diabetes (Roizen et al., 2013); however the occurrence of diabetes was not specified as Type 1 or Type 2 .

Gastrointestinal disorders. One of the more commonly diagnosed gastrointestinal (GI) disorders in adults with DS is gastroesophageal reflux disease (GERD; Cohen, 1999; Kerins et al., 2008; McCarron et al., 2005). Kerins et al. reported $14 \%$ of 141 adults with DS aged 35-65 having GERD. McCarron et al. (2005) cited an increased incidence of obesity as a potential cause. Other GI problems noted to be increased in adults with DS were celiac disease, chronic constipation, and Hirschsprung disease (Bosch, 2003; Cohen, 1999; Crocker, 2006a; Virji-Babul et al., 2007). Additionally, dental problems, including gingivitis, missing teeth, and fissured tongue, are more common in adults with DS (Bosch, 2003; Cohen, 1999; Galley, 2005; Service \& Hahn, 2003; Virji-Babul et al., 2007).

Immune deficiency. Individuals with DS have an increased occurrence of impaired acquired immunity and are potentially candidates for $\operatorname{IgG}$ supplementation in certain circumstances (Bosch, 2003; Cohen, 1999; Galley, 2005; Service \& Hahn, 2003). 
Additionally, carrier status of Hepatitis B is more common in those with DS (Galley, 2005; Kerins et al., 2008).

Mental health disorders. Several mental health disorders other than depression were cited as occurring more frequently in the population with DS. Attention deficit hyperactivity disorder (Cohen, 1999), anxiety (Kerins et al., 2008; Virji-Babul et al., 2007), and autistic spectrum disorders (Bosch, 2003; Cohen, 1999) were reported to have a greater incidence. Additionally, individuals with DS more often display the compulsive behavior associated with obsessive-compulsive disorder, with fewer of the obsessive behaviors than demonstrated by the general population (Bosch, 2003; Cohen, 1999; Galley, 2005).

Musculoskeletal disorders. Osteoporosis is found more frequently in adults with DS than in those of the same age without DS (McCarron et al., 2005; Service \& Hahn, 2003). Osteoarthritis is also increased, as is ligament laxity and atlantoaxial instability which could have debilitating or fatal consequences (Bosch, 2003; Cohen, 1999; Galley, 2005; Kerins et al., 2008; McCarron et al., 2005; Service \& Hahn, 2003; Virji-Babul et al., 2007). The occurrence of having flat feet requiring non-invasive interventions was found higher in a health-study of 62 children with DS (Thomas et al., 2011).

Neurological disorders. As individuals with DS age, new-onset seizures occur more frequently than in the general population (Bosch, 2003; Cohen, 1999; Kerins et al., 2008; McCarron et al., 2005). In fact, adult-onset epilepsy typically occurs in the 30s and 40s in individuals with DS, whereas adult-onset epilepsy in individuals without DS tends to begin in the 60s (Lefter, Costello, McNamara, \& Sweeny, 2011). Additionally, increased seizure activity has been noted as an individual with DS develops worsening AD, which is not as common in the general population (McCarron et al., 2005).

Renal disease. Adults with DS are more frequently diagnosed with hydroureteronephrosis, renal hypoplasia, and vescioureteral reflux (Bosch, 2003; McCarron et al., 2005). Additionally, structural defects of the urinary system, such as hypospadias, are more common (Bosch, 2003).

Reproductive disorder. Males with DS are more likely to have cryptorchidism, and nearly all are infertile (Bosch, 2003; Galley, 2005; Service \& Hahn, 2003). Females are generally able to conceive and maintain a pregnancy, but there is increased gonadal dysfunction, and puberty often occurs earlier than in females without DS (Bosch, 2003; Service \& Hahn, 2003). Females with DS also tend to begin menopause before those without DS, but symptoms may be misdiagnosed as depression or dementia (Bosch, 2003; Service \& Hahn, 2003). 
Respiratory disease. Because of narrowed airways and, often, impaired immune systems, individuals with DS have more frequent respiratory infections (Bosch, 2003; Cohen, 1999; Esbensen et al., 2007; Kerins et al., 2008; McCarron et al., 2005; Service \& Hahn, 2003; Virji-Babul et al., 2007). Obstructive airway disease is also a problem in individuals with DS for the same reasons (Bosch, 2003; Cohen, 1999; Kerins et al., 2008; McCarron et al., 2005; Service \& Hahn, 2003).

Sensory impairments. Structural differences were the primary reason cited for the increased occurrence of hearing loss in individuals with DS, including narrow external auditory canals and frequent otitis media during childhood (Bosch, 2003; Cohen, 1999; Galley, 2005; McCarron et al., 2005; Service \& Hahn, 2003; Virji-Babul et al., 2007). Ocular problems were noted to be increased, including cataracts and keratoconus (Bosch, 2003; Cohen, 1999; Galley, 2005; Kerins et al., 2008; McCarron et al., 2005; Service \& Hahn, 2003; Virji-Babul et al., 2007).

Skin conditions. Persons with DS have been noted to have more extreme dry skin that those without DS (Bosch, 2003; Kerins et al., 2008; Service \& Hahn, 2003). Additionally, fungal infections, alopecia areata, and impetigo are noted more frequently (Bosch, 2003).

Sleep disorders. Obstructive sleep apnea (OSA) is a common occurrence for individuals with DS (Bosch, 2003; Cohen, 1999; Galley, 2005; Kerins et al., 2008; Service \& Hahn, 2003; Virji-Babul et al., 2007). Cohen indicated that obesity could be a cause of the increased frequency of OSA. Other sleep disorders include light sleeping and frequent waking (Bosch, 2003; Cohen, 1999).

\section{Health risks of individuals with Down syndrome}

While the previous section discussed physical and mental health diagnoses for which individuals with DS have a higher risk, the term health risk in this study refers to risky behaviors that can cause or contribute to health problems. Individuals with DS living in the community can potentially be exposed to alcohol, tobacco, and illicit drugs, and may participate in sexual activity (Ailey \& Melich-Munyan, 2010; McGuire \& Chicoine, 2006; White-Scott, Spitalnik, Lunsky, \& Havercamp, 2006).

No extant research was found on incidences or frequencies of these health risks in adults with DS. However, Havercamp, Scandlin, and Roth (2004) found that $17.8 \%$ of 946 adults with a developmental disability in North Carolina reported a history of smoking cigarettes compared to $24.8 \%$ of individuals without a disability. McGillicuddy (2006) determined that $18 \%$ of adults with an intellectual and/or developmental disability (IDD) and a dual mental health diagnosis smoke cigarettes, with the rates increasing as the severity of IDD decreases. Additionally in the same review, it was demonstrated that 
between one-fourth to one-half of adults with IDD consume alcohol regularly, which is less than adults without IDD, but misuse/abuse rates were higher in those with IDD than those without (McGillicuddy, 2006). A similar pattern holds true for the use and misuse of illicit substances, especially marijuana. Those individuals with IDD who misuse alcohol and drugs demonstrate a higher degree of risk factors such as a dual mental health diagnosis, familial problems, or social issues. From a review of literature, Servais (2006) concluded that approximately half of adults with mild to moderate IDD are sexually active, but this number decreases as the severity of the IDD increases.

\section{Health-care utilization by individuals with Down syndrome}

No literature was identified detailing studies on health-care utilization or access by adults with DS specifically; therefore this discussion will begin with research for children with DS and then consider adults with IDD. Schieve, Boulet, Boyle, Rasmussen, and Schendel (2009) determined that children ages 3 through 17 years old with DS utilize health-care services from general practitioners; medical specialists; mental health providers; and physical, occupational, speech, or respiratory therapists significantly more than children without DS. Children with DS visited an outpatient provider three times more often than children without DS, had more frequent hospitalizations (McGrath, Stransky, Cooley, \& Moeschler, 2011), and utilized dental care more often (Allison, Hennequin, \& Faulks, 2000). It was noted that the use of these medical services generally decline with age (Schieve et al., 2009).

In a systematic review, Krahn, Hammond, and Turner (2006) noted that an increased utilization of health-care services was positively associated with greater healthcare needs in individuals with IDD. However, barriers to seeking or receiving care included transportation problems, misunderstanding the severity of illness, inability to locate a provider, African-American race, and lower socio-economic status. Havercamp et al. (2004) reported that adult women with IDD had lower levels of screening for cancers of the breast and cervix; $11.5 \%$ of 415 women had never had a Papanicolaou test (Pap smear), and 26.8\% had never had a mammogram. The age distribution of the entire sample of individuals with IDD ( $\mathrm{n}=946$ ) was $47.5 \%$ respondents $18-34$ years old, $43.1 \%$ 35-54 years old, and $9.4 \% 55$ years or older.

In 2012, according to Sara Weir, the vice president of advocacy and affiliate relations of the National Down Syndrome Society, $80 \%$ of individuals with DS received health-care coverage through Medicaid (Johnson, 2012). Of 3,076 individuals with an IDD who responded to the 1994-1995 National Health Interview Disability Supplement, 45.6\% had Medicaid, 14.0\% had Medicare, and 6.6\% had both. For individuals without an IDD, $8.8 \%$ had Medicaid, $12.8 \%$ had Medicare, and 1\% had both (Birenbaum \& Cohen, 2006). Currently, individuals who carry Medicaid and Medicare should experience no decrease in benefits due to the Patient Protection and Affordable Care Act of 2010 (Public Law 111-148), and in some circumstances, benefits may increase (Medicare.gov, n.d.). 


\section{Summary}

Down syndrome is an IDD that is caused by a third copy of the 21 st chromosome, which originates from one of three different errors of cell division and/or replication. Individuals with DS have a characteristic phenotype, which includes increased susceptibility to or earlier occurrence of specific health problems than in individuals without DS. As with any individual with or without DS, adults with DS can engage in health risks that have the potential to cause or contribute to health problems. In general, adults with DS may utilize health care as much as or more than individuals without DS, but adult women with DS are screened for breast and cervical cancers less than women without DS. Most individuals with DS are covered by Medicaid for health insurance.

\section{Health-Related Quality of Life}

\section{Individuals with Down syndrome}

There is an interest in research on the effect that health conditions have on a person's perception of his/her health and HRQOL (Guyatt, Feeny, \& Patrick, 1993). Because DS is a life-long IDD that has a substantial impact on an individual's life and health, it is reasonable to assess the HRQOL of individuals with DS. However, as mentioned previously, few extant studies have been identified that measure the variables which make up HRQOL of individuals with DS.

Bertoli et al. (2011) identified the prominent association of health and function with an individual's quality of life (QOL) in their study including 518 individuals of all ages with DS in Rome, Italy. A questionnaire was developed which could be answered by the participant with DS or a proxy-respondent if necessary. Bertoli et al. concluded that the HRQOL of older participants with DS was "very poor" (p. 815) because of health problems, limited social relationships, restricted educational and employment opportunities, and lack of independence. However, the investigators did not signify standardized norms by which to compare the data for the individuals with DS, and there was no indication of predetermined or justified ranges of scores that would designate these QOL scores as poor. The investigators who developed this instrument for their particular study did not provide evidence supporting the reliability and validity. Bertoli et al. identified comprehension of some questions by persons with DS as a limitation of the study.

Investigations obtaining information from individuals with DS or other IDDs encounter a substantial challenge because of frequently associated communication and comprehension limitations (Cooper et al., 2009; Smith \& Savage, 2010). Often, these limitations have been avoided by including a proxy-respondent, such as a family member, to answer for the individual with IDD (Cooper et al., 2009; Smith \& Savage, 2010). Often this challenge seems insurmountable. For example, Welke et al. (2007) chose to omit collection of the Medical Outcomes Study Short Form-12 (SF-12), the predecessor 
of the instrument used in the current study, from the adults with DS, $24.8 \%$ of the participants in their study on surgical coronary repair, without discussion of the rationale for the decision.

Measuring the HRQOL of children can also prove complex, but investigators often select instruments designed for parents to provide objective responses (van Gameren-Oosterom et al., 2011; Verstegen et al., 2013). The HRQOL of children with DS in the Netherlands has been assessed in two separate studies using the TNO-AZL Children's Quality of Life questionnaire. This instrument was created for the parental measurement of pain; symptoms of disease or disability; autonomy; and functioning of motor ability, cognition, social aspects, positive emotional aspects, and negative emotional aspects in children (Vogels et al., 1998). The first study investigated behavior and HRQOL of children with DS (van Gameren-Oosterom et al., 2011). These children had lower scores in cognition, social function, independence, and gross motor skills than the comparison norms of children without DS. However, there was no significant difference in physical complaints, regardless of the fact that one or more comorbidity existed in $90 \%$ of the children (van Gameren-Oosterom et al., 2011). Verstegen et al. (2013) focused on the HRQOL of children with DS who had recurrent respiratory infections, finding they had lower HRQOL scores in social functioning, independence, motor skills, and physical well-being subscales (four of the seven assessed) than the control group, which was comprised of children with DS without recurrent respiratory infections (Verstegen et al., 2013).

\section{Individuals with an intellectual and/or developmental disability}

Expanding the confines of the search to include IDD not limited to DS, the HRQOL of women with cerebral palsy (CP) living in a community setting demonstrated that, in spite of their health problems resulting from and in addition to $\mathrm{CP}, 87 \%$ of the respondents claimed to be healthy. This finding is consistent with the general population based on 1993 data from the Behavioral Risk Factor Surveillance System of the Centers for Disease Control and Prevention (Turk, Geremski, Rosenbaum, \& Weber, 1997). Additionally, $34 \%$ of the respondents reported the secondary condition of cognitive impairment, but some proxy-respondents were used in the survey for women who were unable to respond for themselves (Turk et al., 1997).

Brown and Bayer developed a QOL questionnaire for individuals with IDD that could be obtained from self-report (Brown, 1994). However, the subject matter from the questionnaire is more accurately categorized as adaptive behavior as defined in this study and does not inquire about the health of the individual.

Children with prenatal exposure to alcohol participated in a HRQOL study using the Health Utilities Index Mark 3 (HUI3; Stade, Stevens, Ungar, Beyene, \& Koren, 2006) which was administered to both the children and their parents. The HUI3 measures eight domains of HRQOL, "vision, hearing, speech, ambulation, dexterity, emotion, cognition, and pain" (p. 3). The global result was that the HRQOL of the children was significantly 
lower than that of the population norms. The proportions of children with mild, moderate, and severe impairments were $46.8 \%, 32.5 \%$, and $11.2 \%$, respectively. Although the children rated their own HRQOL higher than the parents did, a strong correlation was found between the children's and the parents' responses. However, 33\% of the child participants did not complete the HUI3 due to the degree of their disability (Stade et al., 2006).

\section{Individuals with cognitive impairment}

Considering the dearth of literature on HRQOL of individuals with DS and IDD, the focus was broadened to include research on HRQOL of individuals with a cognitive impairment. The physiological nature of DS includes a level of cognitive impairment that involves a deficiency in receptive and expressive language, memory, and comprehension (Byrne, MacDonald, \& Buckley, 2002). This neurological profile is similar to the neurological profile for individuals with moderate to severe dementia (Sachs et al., 2011); therefore, literature on the HRQOL of individuals with dementia as well as decreased cognitive abilities from causes other than dementia can contribute to this discussion.

A comparison between self- and proxy-report was made by Arlt et al. (2008) on the HRQOL of 100 individuals with dementia, ranging from 48-92 years of age. Arlt et al. noted that self-report in individuals with mild to moderate dementia was accurate and reliable. Additionally, the self-reported HRQOL tended to be higher than the proxyreports.

Jonsson et al. (2006) also correlated self- and proxy-reports regarding adults with dementia, but administered both the generic EuroQol EQ-5D and an Alzheimer-specific QOL instrument to validate responses. The EuroQol EQ-5D is an instrument that examines five domains of HRQOL: "mobility, hygiene, usual activities, pain/discomfort, and anxiety/depression" (Jonsson et al., 2006, p. 50). As discovered by Arlt et al. (2008), self-reported HRQOL was higher than proxy-reported HRQOL. The discrepancy in the self-reported and proxy-reported HRQOL could be attributed to the impaired ability of adults with dementia to interpret and understand questions and express themselves (Jonsson et al., 2006).

Kerner, Patterson, Grant, and Kaplan (1998) were able to validate the Quality of Well-Being Scale (QWB) to measure quality-adjusted life years, a HRQOL utility feature, and found lower scores in individuals with lower cognitive function, indicating diminished HRQOL. However, an observer version of the QWB scale was used for this analysis, and some proxy-respondents participated.

The HRQOL of older adults rehabilitating after a hip fracture indicated that depression and cognitive impairment were associated with lower SF-12 mental component summary scores only (Feng et al., 2010). This study indicates that the SF-12 
surveys were administered in interviews between the individual assessed and a research nurse, so in this circumstance, self-report was obtained.

\section{Summary}

Health-related quality of life has not been quantitatively measured in adults with DS, rather the QOL of adults with DS was presumed to be poor based on the investigation of some characteristics of HRQOL. The HRQOL of children with DS by parental report has been investigated in the Netherlands, finding that HRQOL is influenced by chronic disease, but not directly by DS. Women with CP were found to have a HRQOL that was comparable to population norms, but children with prenatalalcohol exposure scored lower than the population norms. Finally, individuals with moderate to severe dementia that is comparable to the cognitive impairment experienced by individuals with DS were determined reliable providers of information to measure their HRQOL, but reported a higher HRQOL than caregivers. In general, other HRQOL studies for individuals with cognitive impairment not caused by DS resulted in average to below-average HRQOL.

\section{Self-Report Versus Proxy-Report}

The concept of self-determination, or autonomy, has become a priority in the research, health care, education, and services of individuals with IDD (Wehmeyer \& Bolding, 2001). Therefore, it would seem appropriate to address the quandary of collecting subjective data, such as HRQOL, from proxy-respondents based on objective opinions and observations.

Radbruch et al. (2000) and Seymour et al. (2001) encountered the challenges of administration of the SF-12 and the Short Form-36 Health Survey Questionnaire (SF-36) respectively to individuals with cognitive impairment. They reported finding increased missing data and decreased internal consistency. Fujiura (2012) acknowledged the complication of impaired verbal expression and comprehension for individuals with IDD. However, instruments utilized in studies of HRQOL could be adapted to accommodate differences in communication and understanding for individuals with IDD, or an instrument could be substituted that was created specifically for use in this population (Graff, 2010).

Fujiura and Behrens (2011) recognized and attempted to address this challenge by adapting the SF-12 to create a subjective survey of adults with IDD, indicating that the success of this attempt was limited, and further research was needed. Arlt et al. (2008) suggested that a scrupulous understanding of the subjective health reports of an individual unable to thoroughly answer for himself/herself may be obtained from input from the individual, the caregiver, and the health-care provider. 
When measuring a subjective concept based on the objective observations and opinions of others, self-determination is sacrificed. Researchers, health-care providers, and educators need to find methods to gain subjective information from the individual despite communication and/or cognitive challenges.

\section{Adaptive Behavior}

As discussed previously, adaptive behavior includes actions or skills necessary for an individual to assimilate and adjust to his/her environment on a daily basis. Limitations in adaptive behavior is one of three components necessary in the diagnosis of intellectual disability, with the other two being limitations in intellectual functioning and onset of the disability prior to or shortly after birth (Schalock et al., 2010). Brown (1994) found that increased adaptive behavior skills, including reading, writing, cooking, and getting out into the community improved the QOL of individuals with DS.

The Vineland Adaptive Behavior Scales, Second Edition (Vineland-II), is one standardized instrument used to assess adaptive behavior in individuals for the purposes of diagnosing individuals with IDD and other physical, behavioral, and psychological conditions; determining that individuals meet qualification criteria for interventions; monitoring progress or changes from an individual's baseline to evaluate interventions; and developing individualized, comprehensive programs of intervention (Sparrow, Cicchetti, \& Balla, 2005). Because the assessment of adaptive behavior contributes to special education and early intervention goals, considerable research exists for the adaptive behavior of the pediatric population with DS. The studies that focus on adaptive behavior of adults with DS do so in the evaluation of mental deterioration, dementia, or AD (Adams \& Oliver, 2010; Lott et al., 2012).

Along with other neuropsychological instruments to measure executive function, dementia, receptive language, and behavior, Adams and Oliver (2010) used the first edition of the Vineland Adaptive Behavior Scales (VABS), the predecessor to the Vineland-II which is used in this study, to assess adults with DS with early cognitive deterioration (mean age $=48.9$ ) and without early cognitive deterioration (mean age $=42.3$ ) in a longitudinal study. No significant differences were reported between the two groups at any point or in each group at the 8-month or 16-month follow-up assessments.

However, Lott et al. (2012) did identify differences in VABS scores in a secondary analysis of a longitudinal study to investigate differences in cognitive deterioration and seizure activity in adults with DS. All participants in the study had dementia, including 24 participants who had seizures (mean age $=50.38$ ) and 29 who did not have seizures (mean age $=50.86$ ). Over the two years of the study, the mean scores for each subdomain of the VABS were significantly lower in the group with seizures than in the group without seizures (differences of 12.7 points for communication, 26.7 points for daily living, 17.4 points for socialization, and 12.0 points for motor skills.) 
Adaptive behavior is a concept that is integral to the diagnosis of IDD, and it is commonly measured during the school years of children with IDD. Improved adaptive behavior has been associated with improved QOL of individuals with DS. Studies involving adults with DS have attempted to associate adaptive behavior with the progression of dementia with mixed results.

\section{Summary}

Individuals with DS have a greater risk of specific health problems and may experience other health problems earlier than individuals without DS. Adults with DS may increase their risks of health problems by engaging in unhealthy behaviors. General health-care utilization of adults with DS may be higher than that of adults without DS, and most are covered by Medicaid. However, cancer screening is often neglected in women with DS.

Studies have not been done that quantify the HRQOL of adults with DS, but those focusing on children with DS, women with $\mathrm{CP}$, children with prenatal-alcohol exposure, and adults with dementia determined HRQOL scores to be average to below-average. All of these studies utilized at least some proxy-respondents, which dilutes the accuracy of HRQOL since this is a fundamentally subjective concept.

Adaptive behavior, which is generally evaluated in individuals with IDD during childhood, contributes to improved QOL in adults with DS. Other studies on the adaptive behavior of adults with DS have been contradictory, but it is theorized that adaptive behavior decreases with the progression of cognitive decline or dementia. 


\section{CHAPTER 3. METHODOLOGY}

The purpose of this study was to examine the health-related quality of life (HRQOL) and factors associated with the HRQOL of adults with Down syndrome (DS) as reported by adults with DS and their caregivers. The design, sample, setting, instruments, procedure, and data analysis of this study are explained in this chapter.

\section{Research Design}

A mixed-methods design was used in this descriptive, correlational, crosssectional study that included established surveys, investigator-developed questionnaires, and a semi-structured interview administered during face-to-face meetings. The data were collected at multiple sites throughout the southeastern U.S.

The data-transformation variant of the convergent mixed-methods design was selected, which permitted quantification of qualitative data and validation of quantitative data and also facilitated comparisons, contrasts, and analysis using quantitative data from surveys and interviews (Creswell \& Plano Clark, 2011). The adults with DS shared their perception and understanding of their health problems, health risks, and health-care utilization to validate caregiver-report.

\section{Sample}

Protection for human subjects. The University of Tennessee Health Science Center Institutional Review Board (UTHSC IRB) approved the study and related procedures to recruit adults with DS. Adults with DS are classified as a vulnerable population because DS is an intellectual and/or developmental disability (IDD; Betz \& Jones, 2010; Smith \& Savage, 2010). Participation in the study also included an individual who is the, or one of the most familiar companions of the adult with DS and has known the adult with DS longer than six months (caregiver).

The UTHSC IRB Standard Operating Procedures for Informed Consent outlines the necessity to obtain consent from a legally authorized representative if an individual is unable to provide consent due to inadequate decision-making capacity (University of Tennessee Health Science Center Institutional Review Board [UTHSC IRB], 2010). The study application to the UTHSC IRB included definitions of legally authorized representatives from each state from which participants were recruited.

Based on ethics guidelines for research with individuals with IDD (Dalton \& McVilly, 2004; UTHSC IRB, 2010), informed consent for participation in the study was obtained from adults with DS if they did not have a designated legal guardian or conservator, with questions included after explanation of the study to verify the 
comprehension and capacity for consent of the adult with DS (see Appendix A). For adults with DS who had a designated legal guardian, consent was obtained from the legal guardian to permit the adult with DS to participate in the study. In these circumstances, the adults with DS provided assent to participate in the study. Again, questions were included after explanation of the study to verify comprehension and capacity for assent. For all participant dyads, informed consent was also obtained from the caregivers for their participation (see Appendix B).

Recruitment. According to Thorndike's Rule of Thumb regarding minimal sample size to establish statistical power using multiple regression (Prescott, 1987), recruitment of 60 to 80 participant dyads (adult with DS and his or her caregiver as a single dyad) was the goal for this study. Face-to-face meetings were conducted in the southeastern U.S., including areas surrounding Birmingham, Alabama; Chattanooga, Tennessee; Gulfport, Mississippi; Memphis, Tennessee; Mobile, Alabama; Nashville, Tennessee; and Pensacola, Florida. Based on data from the 1997-2005 National Health Interview Survey, the ethnic distribution of children with DS (79.0\% white, $13.1 \%$ black, and $7.9 \%$ all other ethnicities) differs from that of children without DS or an IDD slightly (77.1\% white, $15.6 \%$ black, and $7.2 \%$ all other ethnicities; Schieve, Boulet, Boyle, Rasmussen, \& Schendel, 2009). Ethnic distribution similar to this national distribution of individuals with DS was a goal of this study.

Study participants were approached through word-of-mouth (e.g., network or snowball sampling) as well as via mailings and emails to persons on distribution and membership lists available through DS support/advocacy groups, health-care facilities with a focus on adults with DS, Special Olympics activities, and regional centers that provide services to individuals with DS. Recruitment flyers were also displayed in these facilities with contact information (see Appendix C). A gift card to a national retail chain in the amount of $\$ 10.00$ was offered to each adult with DS and each caregiver as an incentive to participate in the study.

Participant criteria. Eligibility for the study was determined via telephone and/or email communication. Adults with DS or their caregivers responded to information about the study, and criteria were assessed at that time authorized by a waiver of consent to obtain personal information from UTHSC IRB.

Inclusion criteria for adults with DS included:

1) age 18 years or older;

2) diagnosis of DS;

3) residing in a community dwelling, including own home, friend's home, parent's/family member's home, and group home;

4) the ability to understand and speak English; and

5) the ability to understand the study and provide consent/assent to participate. 
The exclusion criterion for adults with DS was the inability to appropriately answer questions designed to ascertain comprehension of informed consent or assent.

Inclusion criteria for the caregivers were individuals with:

1) a close relationship (spouse, family, friend, or paid caregiver) for longer than six months with the adult with DS;

2) contact with the adult with DS (in person or via telephone or electronic communication) at least once a month;

3) the ability to understand and speak English; and

4) the ability to understand the study and provide consent to participate.

The exclusion criterion for caregivers was lack of familiarity with health and adaptive behaviors of the adult with DS.

\section{Quantitative instruments}

Demographic characteristics of both the adult with DS and the caregiver were collected. Additionally, data regarding HRQOL, health problems, health risks, healthcare utilization, and adaptive behavior of the adult with DS were obtained. In this study, the associations between the HRQOL of adults with DS and factors potentially contributing to their HRQOL (demographic characteristics, health problems, health risks, health-care utilization, and adaptive behavior) were examined.

Demographic Form. A demographic form was developed by the investigator and completed by the caregiver to obtain information about the caregiver and the adult with DS. Questions solicited information about age, gender, ethnicity, marital status, education, and employment/daily activity of both the caregiver and the adult with DS based on variables assessed in other studies on the HRQOL of individuals with IDD (Arlt et al., 2008; Turk, Geremski, Rosenbaum, \& Weber, 1997; van Gameren-Oosterom et al., 2011; Verstegen et al., 2013). The form asked about the frequency of interaction between the caregiver and the adult with DS to help define the nature of this relationship, as well as the living arrangement of the adult with DS to contribute information about the level of independence of the adult with DS (see Appendix D).

Health Questionnaire. A 50-item general Health Questionnaire, adapted from a questionnaire used in an earlier study on health (Graff, 04/16/14; Graff, Engle, \& Pruett, 2003) was completed by the caregiver as proxy-respondent on behalf of the adult with DS. In the current study, the contribution of the health of adults with DS to HRQOL was examined (see Appendix E).

The Health Questionnaire Section I, Health Problems consists of 32 items with yes/no responses and includes general health problems, including those more commonly 
encountered by adults with DS (Cohen, 1999; Esbensen, 2010). These items focus on symptoms and their effects rather than specific diagnoses (Wilson \& Cleary, 1995). Section II, Health Risks, also contains items with yes/no responses regarding risky behaviors that can contribute to health problems, such as sexual activity and use of alcohol, tobacco, and illicit drugs, which can be exhibited by individuals with DS and other IDD (Ailey \& Melich-Munyan, 2010; McGuire \& Chicoine, 2006; White-Scott, Spitalnik, Lunsky, \& Havercamp, 2006). Sections I and II were composed to solicit health information collected in HRQOL studies of children with DS (van GamerenOosterom et al., 2011; Verstegen et al., 2013), women with cerebral palsy (Turk et al., 1997), and adults with cognitive impairment (Arlt et al., 2008). The responses from sections I and II were coded with a zero for "no" and a 1 for "yes". Although sums were calculated for the total number of health problems and total number of health risks, an overall score was not calculated from the Health Questionnaire; rather the results from the individual items were correlated with the data on HRQOL.

Section III of the Health Questionnaire, Health-Care Utilization, has 11 items regarding the frequency of medical encounters and screenings and are based on guidelines for adults with DS (Cohen, 1999) and previous research of the HRQOL of individuals with IDD (Arlt et al., 2008; Turk et al., 1997; van Gameren-Oosterom et al., 2011; Verstegen et al., 2013). These 11 items were scored as follows: 1="in the last month", 2="in the last year", $3=$ "in the last 2 years", and 4="other" as responses to indicate length of time since last visit or procedure. Item 48 asked if there is a regular primary health-care provider and responses were coded with a zero for "no" and a 1 for "yes". Item 49 asked what type of insurance the adult with DS has, and responses were entered as follows: $0=$ "none", 1="private", 2="Medicaid", 3="Medicare", and 4="other". Item 50 asked about potential barriers to care, and responses were coded as: $0=$ "none", $1=$ "does not know who to go to", 2="does not know where to go", $3=$ "transportation problems", 4="work problems", 5="appointments take too much time", 6="does not like or trust the health-care provider", 7="feels he/she is not understood or respected", 8="is afraid of the health-care provider", 9="clinics are too far away, inconvenient", 10="does not have enough help from others", 11="does not have enough support from others", $12=$ "does not want to think about health problems", 13="money problems", and $14=$ "other".

The Health Questionnaire was used to guide the development of the Health Interview Guide to ascertain information about the health problems, health risks, and health-care utilization of adults with DS in an interview format. The interviews are discussed in detail in the Qualitative Interviews section of this chapter.

QualityMetric Short Form-12v2 Health Survey. The acute recall form of the QualityMetric Short Form-12v2 Health Survey (SF-12v2) includes items about an individual's perception of his/her health and activity limitations within the previous seven days (Ware et al., 2007). This 12-item survey instrument measures eight scales of one's health profile which include physical functioning, role-physical (functioning in one's usual role in association with one's physical health), bodily pain, general health, vitality, 
social functioning, role-emotional (functioning in one's usual role in association with one's emotional health), and mental health (Saris-Baglama et al., 2011; Ware et al., 2007). The survey items are in a 3- to 5-point Likert-type scale format. The responses result in overall physical component summary (PCS) and mental component summary (MCS) scores ranging from zero to 100, with the levels of physical and mental health improving as the score increases (Ware et al., 2007). QualityMetric Health Outcomes ${ }^{\mathrm{TM}}$ Scoring Software 4.5 was utilized to obtain scale and component summary scores from the completed surveys due to complex algorithms.

Psychometric properties of the SF-12v2 were calculated for a sample of 20,699 participants in the 2003-2004 medical expenditure panel survey, a national probability survey. The Mosier alpha determined high internal consistency for weighted PCS (.88) and MCS (.82). Intraclass correlational coefficients (ICC) determined test-retest reliability in participants who had stable perceived health scores. Individuals who were physically stable $(n=4,891)$ had ICC scores of .78 for high reliability; those who were mentally stable $(n=4,960)$ had ICC scores of .60 for moderate reliability (Cheak-Zamora, Wyrwich, \& McBride, 2009). Wells (2010) administered the SF-12v2 to 277 adults without DS or IDD who were at least 65 year old and determined the Cronbach's alphas to be .89 for the PCS and .87 for the MCS. Wells does not indicate consideration of the algorithms in the calculation of these properties of the instrument.

The acute recall form of the SF-12v2 was selected based on impaired long-term explicit memory, or an individual's memory of experiences or specific events, that is found in individuals with DS (Lott, 2012; Vicari, Bellucci, \& Carlesimo, 2000). In a study of individuals with asthma who did not have DS or IDD, Keller et al. (1997) compared the standard recall (one month) version of the Short Form-36 (SF-36) Survey, the precursor to the first version of the Medical Outcomes Study Short Form-12 (SF-12), to the acute recall form of that instrument. The measurements of physical and mental health constructs in this study were comparable, and the few differences found were attributed to the perception of acute changes in diseases and symptoms. The most noteworthy inconsistencies were found in norm-based scoring comparisons, which have been corrected by creating norms specifically for the SF-12 and SF-12v2 acute recall forms (Keller et al., 1997). Regardless, raw and norm-based scoring comparisons do not contribute to the research questions in the current study; therefore, they were not included in analysis, but rather they provided an overview of the scales.

Ware, Kosinski, and Keller (1996) confirmed preliminary test-retest reliability after creating the SF-12 from the SF-36, a 36-item measure of health and HRQOL. The SF-12 was created by selecting two items each from the physical functioning and mental health scales, since these best predict physical health and mental health respectively, and one item from each of the other six scales through cross-validation (Gandek et al., 1998). The preliminary test was administered to individuals without IDD. In the United States, the PCS had a coefficient of 0.890 and the MCS had a coefficient of 0.760 (Ware et al., 1996). For both SF-12 component summary measures, the mean change in scores between the first test and the retest was less than one point, and $85.3 \%$ of the scores for the retest were within the $95 \%$ confidence interval of the first test (Ware et al., 1996). 
Fujiura and Behrens (2008) tested an adapted version of the SF-12 to measure the HRQOL of individuals with IDD. The adapted instrument, which simplified the language and response format, was used to test the understanding of question items and concepts of ten adults with mild to moderate IDD using probe questions after each item. Findings in this study were inconclusive and necessitated further adaptation for satisfactory results. The SF-12 was selected for this study due to its prominent use in HRQOL research among other populations.

Jones, Dagnan, and Ruddick (1997) assessed the HRQOL of 71 adults with IDD living in community homes in the United Kingdom (UK) using the SF-36 due to the wide use and acceptance of the instrument in the U.S. and the UK. The SF-36 was completed by staff members who were key to the care of each individual. The Cronbach's alphas were calculated for each subscale of the SF-36, as well as for the total score: physical functioning $=0.94$, role-physical $=0.91$, bodily pain $=0.88$, general health $=0.87$, vitality $=0.81$, social functioning $=0.33$, role-emotional $=0.93$, mental health $=0.71$, and total $=0.92$. The Cronbach's alphas for each subscale were satisfactory except for social functioning (Jones et al., 1997).

Ruddick and Oliver (2005) used the SF-36 as a model to create an adapted instrument, the Health Status Interview Schedule, to measure HRQOL in adults with IDD because of the use and acceptance of the SF-36. The SF-36 was also chosen because of the publication of norms based on numerous studies and samples. The results did not produce adequate reliability and consistency for the eight scales that were modeled after the SF-36.

There is no literature in the English language to support the use of the SF-12v2 to ascertain the HRQOL of adults with DS or adults with IDD. Swedish and English versions of the SF-36 have been used to assess the HRQOL of caregivers and family members of individuals with DS and IDD respectively (Caldwell, 2008; Hedov, Anneren, \& Wikblad, 2000). Hedov et al. used the Swedish version of the SF-36 to determine the HRQOL of parents of children with DS. They reported a Cronbach's alpha coefficient for each domain ranging from 0.72 for social functioning and role-emotional to 0.92 for bodily pain. Caldwell did not report estimates of reliability.

When administering the SF-12v2 to adults with DS, the 3- to 5- response levels were separated, giving only two responses at a time without changing the response format or item responses. This was planned because the response choices were presented verbally, which could exacerbate a decreased understanding and the recognized bias that many individuals with IDD have interpreting degree in these types of questions (Finlay \& Lyons, 2001). For example, questions about limitation of activity have three response levels: "yes, limited a lot", "yes, limited a little", and "no, not limited at all". The investigator provided these three options two at a time, stating, "yes, limited" or "no, not limited at all". If the respondent stated "yes", the investigator then provided the two yes options: "yes, limited a lot" or "yes, limited a little". 
Vineland Adaptive Behavior Scales, Second Edition. Adaptive behavior, or the actions that enable a person to interact with others and function within his/her environment (Woolf, Woolf, \& Oakland, 2010), was assessed using the Vineland Adaptive Behavior Scales, Second Edition (Vineland-II) Parent/Caregiver Rating Form and was completed by the caregivers. This instrument is designed to measure baseline and changes in adaptive behavior from childhood up to the age of 90 years (Sparrow, Cicchetti, \& Balla, 2005).

The Vineland-II is organized into three primary domains and each domain is further divided into three subdomains for individuals seven years of age and older (Sparrow et al., 2005). The domains with subdomains in parentheses are: communication (receptive, expressive, and written), daily living skills (personal, domestic, and community), and socialization (interpersonal relationships, play and leisure time, and coping skills). Scoring of the responses in these three domains results in the adaptive behavior composite score, which indicates overall adaptive behavior (Sparrow et al., 2005; Wilson, Jordan, \& Kras, 2010). The adaptive behavior can then be ranked from low to high based on the composite score. The separate domain and subdomain scores indicate an individual's aptitude in comparison with normative data (Wilson et al., 2010). In this study, the adaptive behaviors of adults with DS were examined as potential contributing factors to HRQOL. The Vineland-II ASSIST ${ }^{\mathrm{TM}}$ Scoring and Reporting System Software was utilized to determine composite, domain, and subdomain scores from the completed surveys.

The subdomain v-scale scores range from 1 to 24 , and have a mean of 15 , a standard deviation of 3 , and are standardized according to the age of the individual. Vscale scores of 21 and above indicate high adaptive level for that subdomain, 18-20 is moderately high, 13-17 is adequate, 10-12 is moderately low, and 9 or less is low (Sparrow et al., 2005).

Overall adaptive behavior and domains can be represented in standard scores, which also compare an individual to his or her age group. The means of standard scores are 100, they have standard deviations of 15 and ranges of 20 to 160, and they are in a normal distribution curve. A standard score of 130 or higher indicates high adaptive level for the adaptive behavior composite or individual domain, 115-129 is moderately high, 86-114 is adequate, 71-85 is moderately low, and 70 or less is low (Sparrow et al., 2005).

For the normative sample of 3695 individuals from birth to the age of 90 in the U.S., the internal consistency reliabilities of the adaptive behavior composite and most domains are above .90 and are above .75 for $75 \%$ of the subdomains (CommunityUniversity Partnership for the Study of Children, Youth, and Families, 2011; Wilson et al., 2010). The test-retest reliabilities are above .85 for almost all adaptive behavior composite, domain, and subdomain scores (Wilson et al., 2010). For individuals with cognitive delay, the mean of the adaptive behavior composite score was two standard deviations below the average of the normative sample (Community-University Partnership for the Study of Children, Youth, and Families, 2011). 
Concurrent validity correlations with the Vineland-II's predecessor, the Vineland Adaptive Behavior Scales (VABS) approximate .90 (Wilson et al., 2010). The VABS has a Cronbach's alpha coefficient of 0.98 for individuals with intellectual disabilities.

Dressler, Perelli, Feucht, and Bargagna (2010) used the VABS (adapted Italian Version) to investigate associations between the adaptive behavior, health, and cognitive ability of individuals with DS who resided in Italy and were between the ages of 4 to 52 years. Dressler et al. reported that individuals with DS continue to develop adaptive behaviors throughout the life-span.

\section{Qualitative interviews}

A semi-structured interview was conducted with each adult with DS to explore his/her health problems, health risks, and health-care utilization. The questions were developed to avoid acquiescence with yes/no questions, a recognized bias in adults with IDD (Finlay \& Lyons, 2001). The interview was administered following an interview schedule, the Health Interview Guide (see Appendix F), which was developed to encourage the adult with DS to provide detail, and questions were included to identify and discourage acquiescence (Lloyd, Gatherer, \& Kalsy, 2006). Data from the interviews were content analyzed and transformed into quantitative data to facilitate statistical comparison with the data from the Health Questionnaire completed by the caregiver.

Conversation concerning specific health problems and risks that arose during an interview with the adult with DS were initially coded as follows for descriptive purposes: specific health problems and risks not mentioned by the adult with DS were coded as "0"; specific health problems and risks that the adult with DS identified or claimed to have were coded as "1"; specific health problems and risks he/she denied having were coded as "2". For analysis, specific health problems or risks that were denied (previously coded as "2") were recoded as "0" to create dichotomous variables with the values of "no/not mentioned" $=0$ or "yes" $=1$ to facilitate comparison with the answers of "no" $=0$ and "yes"=1 from the Health Questionnaires completed by the caregivers.

For descriptive purposes, health-care utilization variables were initially coded as $0=$ "not mentioned", 1="mentioned without time frame", 2="never", $3=$ "longer than 2 years", 4="in the last 2 years", 5="in the last year", and 6="in the last month". For analysis to compare and contrast these answers with those provided by caregivers, the variables were recoded to $0=$ "not mentioned/never", 1="mentioned without time frame/longer than 2 years", 2="in the last 2 years", 3="in the last year", and 4="in the last month".

\section{Sources of data}

Table 3-1 presents the variables to be measured in this study. The respondent who was the source of the data is noted for each variable. 
Table 3-1. Sources of data from adults with Down syndrome and their caregivers

\begin{tabular}{|c|c|c|}
\hline \multirow[b]{2}{*}{ Variables } & \multicolumn{2}{|c|}{ Respondents } \\
\hline & Adult with DS & Caregiver \\
\hline $\begin{array}{l}\text { HRQOL of adult with DS } \\
\text { PCS score } \\
\text { MCS score }\end{array}$ & $\mathrm{SF}-12 \mathrm{v} 2$ & $\begin{array}{l}\text { SF-12v2 by } \\
\text { proxy }\end{array}$ \\
\hline $\begin{array}{l}\text { Demographics for adult with DS } \\
\text { Age } \\
\text { Gender } \\
\text { Ethnicity } \\
\text { Marital status } \\
\text { Education } \\
\text { Employment } \\
\text { Daily activity } \\
\text { Living arrangement }\end{array}$ & & $\begin{array}{l}\text { Demographic } \\
\text { Form }\end{array}$ \\
\hline $\begin{array}{l}\text { Demographics for caregiver } \\
\text { Age } \\
\text { Gender } \\
\text { Ethnicity } \\
\text { Marital status } \\
\text { Education } \\
\text { Employment } \\
\text { Relationship with adult with DS } \\
\text { Frequency of interaction with adult with DS }\end{array}$ & & $\begin{array}{l}\text { Demographic } \\
\text { Form }\end{array}$ \\
\hline $\begin{array}{l}\text { Health of adult with DS } \\
\text { Health problems } \\
\text { Health risks } \\
\text { Health-care utilization }\end{array}$ & $\begin{array}{l}\text { Health Interview } \\
\text { Guide }\end{array}$ & $\begin{array}{l}\text { Health } \\
\text { Questionnaire } \\
\text { by proxy }\end{array}$ \\
\hline $\begin{array}{l}\text { Adaptive behavior of adult with DS } \\
\text { Adaptive composite score } \\
\text { Communication } \\
\text { Daily living skills } \\
\text { Socialization }\end{array}$ & & Vineland-II \\
\hline
\end{tabular}




\section{Procedure}

The study and procedure were explained to the adult with DS and the caregiver together, questions were answered, and consents/assents were obtained. The adult with DS and the caregiver were then separated. The caregiver was asked to complete the questionnaires and surveys in a nearby area that was not visible to the adult with DS to promote privacy and confidentiality of responses unless the adult with DS or caregiver was not comfortable with this positioning or if the absence or lack of visibility of the caregiver contributed to less open responses and observable discomfort from the adult with DS.

The investigator remained with the adult with DS and began the semi-structured interview. The interview was audio-recorded and the recorder was visible to the adult with DS and the researcher. The investigator asked the adult with DS the open-ended questions with the goal of eliciting personal thoughts and perspectives, therefore the caregiver was asked to not comment or contribute during this interview portion. The questions were asked using the level of language and terminology expressed by the adult with DS to provide context of understanding and to confirm comprehension of the topic (Brown, Taylor, \& Matthews, 2001).

The SF-12v2 was then verbally administered to the adult with DS. During data collection, the researcher carefully monitored and observed the individual with DS. If the respondent appeared to have difficulty comprehending the questions, the researcher repeated the question and response options with clarification if necessary.

The caregiver completed the SF-12v2 as a self-administered survey and as proxyrespondent for the adult with DS. This approach asked that the caregiver answer the questions as he/she believed the adult with DS felt and/or thought. The caregiver also completed the Health Questionnaire as a proxy-respondent for the adult with DS after being instructed to answer it as factually as possible based on the caregiver's own understanding and interpretation of the questions. For caregivers who had difficulty comprehending the questions, assistance was provided. These self-administered instruments were reviewed for completeness and clarifications with the caregiver before ending the data collection session. The caregiver was discouraged from influencing the adult with DS during the semi-structured interview and while the SF-12v2 was administered to the adult with DS. Caregivers were also discouraged from being influenced by the adult with DS when the caregiver completed the SF-12v2 and Health Questionnaire. Caregivers also completed the demographic form and the Vineland-II but had the opportunity to consult with the adult with DS to assure accuracy for these two instruments.

\section{Data analysis}

Qualitative data were transcribed and coded, identifying codes related to items in the Health Questionnaire. Intercoder agreement was established by separated individual 
coding of $20 \%$ of the interviews by a second investigator (Creswell \& Plano Clark, 2011). These codes were then transformed to quantitative data (quantitized) and included in analysis using International Business Machine Corporation Statistical Package for Social Sciences (SPSS) version 20 to enable inferential analysis with quantitative data obtained from the caregivers (Sandelowski, Voils, \& Knafl, 2009).

Quantitative data were also analyzed using SPSS. Statistical procedures included descriptive statistics; paired samples $t$ tests; independent samples $t$ tests; Pearson's product-moment, Point-biserial, and Spearman's rho correlation analysis; McNemar tests; Kruskal-Wallis tests, and multiple linear regression analysis.

\section{Specific aim one}

The first specific aim was to measure the HRQOL of adults with DS. The following research question relates to the first specific aim:

Research Question 1 What is the HRQOL of adults with DS as reported by:

a) adults with DS?

b) caregivers of adults with DS?

The HRQOL was measured using the SF-12v2. Descriptive statistics including measures of central tendency and variance were used to characterize responses from the adults with DS (question 1a) and responses from the caregivers of adults with DS (question 1b).

\section{Specific aim two}

The second specific aim was to compare the HRQOL data obtained via self-report to that obtained via caregiver-report. The following research question relates to the second specific aim:

Research Question 2 What are the associations between the HRQOL of adults with DS as reported by adults with DS and their caregivers?

The SF-12v2 responses from adults with DS were compared with the SF-12v2 responses from caregivers of adults with DS using Pearson product-moment correlation to examine associations and paired samples $t$ tests to analyze similarities and differences in their responses. 


\section{Specific aim three}

The third specific aim was to learn more about the health of adults with DS obtained via self-report compared to that via caregiver-report. The following research question relates to the third specific aim:

Research Question 3 What are the associations between health problems, health risks, and health-care utilization of adults with DS as reported by adults with DS and their caregivers?

The qualitative data from the semi-structured interviews of adults with DS that were quantitized were compared with the data from the Health Questionnaire provided by caregivers. Point-biseral correlational analyses were then conducted on the transformed data collected from adults with DS and the data collected from caregivers using the Health Questionnaire to determine associations, and McNemar tests analyzed similarities and differences.

\section{Specific aim four}

The fourth specific aim was exploratory in nature and was to examine the association of the HRQOL of adults with DS to the health, adaptive behavior, and demographic characteristics of adults with DS. The following research question relates to this aim:

Research Question 4 What are the factors (health problems, health risks, healthcare utilization, adaptive behavior, and demographic characteristics) that are associated with the HRQOL of adults with DS as reported by:
a) adults with DS?
b) caregivers of adults with DS?

All characteristics from the demographic form; health problems, health risks, and health-care utilization quantitized from the semi-structured interviews of adults with DS; health problems, health risks, and health-care utilization from the Health Questionnaire provided by the caregivers; and Vineland-II adaptive behavior composite, domain, and subdomain scores were each analyzed for association with the PCS scores and the MCS scores from the SF-12v2 completed by the adults with DS using Pearson productmoment, Point-biseral, or Spearman's rho correlational analysis or Kruskal-Wallis test (question 4a). All characteristics from the demographic form; health problems, health risks, and health-care utilization quantitized from the semi-structured interviews of adults with DS; health problems, health risks, and health-care utilization from the Health Questionnaire provided by the caregivers; and Vineland-II adaptive behavior composite, domain, and subdomain scores were each analyzed for association with the PCS scores and the MCS scores from the SF-12v2 completed by caregivers using Pearson product- 
moment, Point-biseral, or Spearman's rho correlational analysis or Kruskal-Wallis test (question $4 b$ ).

Findings from these analyses identified variables to be entered into multiple linear regression models if they demonstrated a significance of .2 or less. Multiple regression analysis was conducted to identify variables that best predicted PCS and MCS scores of adults with DS obtained via self-report and PCS and MCS scores of adults with DS obtained via caregiver-report. 


\section{CHAPTER 4. RESULTS}

The purpose of this study was to examine the health-related quality of life (HRQOL) and factors associated with the HRQOL of adults with Down syndrome (DS) as reported by adults with DS and their caregivers. This chapter presents the results of the analysis.

\section{Description of the Sample}

The characteristics of the 60 adults with DS and their 60 caregivers who participated in this study are presented in Tables 4-1, 4-2, and 4-3. The ages of the adults with DS ranged from 18 to 61 years old, with a mean of 30.83 years (Table 4-1). There were 30 female adults with DS and 30 male adults. Adults with DS lived in Tennessee (53.3\%), Alabama (38.3\%), Mississippi (6.7\%), and Florida (1.7\%). The sample of adults with DS was Caucasian (91.7\%), African-American (6.7\%), and AmericanHispanic (1.7\%). None of the participants were married, although one man was engaged. The highest level of education completed by most adults with DS was high school graduation with a diploma or certificate of completion $(68.3 \%)$, followed by some high school (15\%), less than 7 th grade $(8.3 \%)$, some college $(6.7 \%)$, and some junior high school $(1.7 \%)$. Four more of the high school graduates were enrolled in a post-secondary program that had not yet begun at the time of the data collection sessions, bringing the total of those in a college program or post-secondary vocational training school to eight $(13.3 \%)$ at the time of this discussion.

Forty percent of adults with DS were employed, either part time or full time, but more were unemployed (Table 4-1). Ten percent of the entire sample of 60 adults with DS worked in an independent working situation, such as a restaurant or a retail establishment (Table 4-2). The caregivers selected all daily activities that each adult with DS was involved in, and approximately one-third worked in a paid part-time job, one-third volunteered, and one-third attended a day program.

Most adults with DS (96.7\%) lived with a parent or relative, but one woman with DS lived with a friend who was her caregiver, and one man lived in his own home with housemates and had a supported-living staff (Table 4-3). This man was the only adult with DS who did not have daily face-to-face contact with his respondent caregiver, rather they saw each other one to two times per week and had telephone, text, or email contact three to four times per week.

The 60 caregivers who participated in the study ranged in age from 44 to 79 years of age with a mean age of 60.6 years (Table 4-1). They were primarily female (90\%), and lived in the same states as the adult with DS for whom they act as caregiver. Caregivers were Caucasian (91.7\%), African-American (6.7\%), and Hispanic (1.7\%). The majority of the caregivers were married (68.3\%). 
Table 4-1. Demographic characteristics of adults with Down syndrome and their caregivers $(\mathrm{N}=120)$

\begin{tabular}{|c|c|c|}
\hline Characteristics & $\begin{array}{l}\text { Adult with DS } \\
(n=60)\end{array}$ & $\begin{array}{c}\text { Caregiver } \\
(\mathrm{n}=60)\end{array}$ \\
\hline \multicolumn{3}{|l|}{ Age in years } \\
\hline $\mathrm{M}(\mathrm{SD})$ & $30.83(9.61)$ & $60.6(7.18)$ \\
\hline Range & $18-61$ & 44-79 \\
\hline Median & 27.5 & 60.0 \\
\hline \multicolumn{3}{|l|}{ Gender, n(\%) } \\
\hline Female & $30(50.0)$ & $54(90.0)$ \\
\hline Male & $30(50.0)$ & $6(10.0)$ \\
\hline \multicolumn{3}{|l|}{ State of residence, $\mathrm{n}(\%)$} \\
\hline Alabama & $23(38.3)$ & $23(38.3)$ \\
\hline Florida & $1(1.7)$ & $1(1.7)$ \\
\hline Mississippi & $4(6.7)$ & $4(6.7)$ \\
\hline Tennessee & $32(53.3)$ & $32(53.3)$ \\
\hline \multicolumn{3}{|l|}{ Ethnicity/race, n(\%) } \\
\hline Black or African-American & $4(6.7)$ & $4(6.7)$ \\
\hline Hispanic or Latino & $0(0)$ & $1(1.7)$ \\
\hline White & $55(91.7)$ & $55(91.7)$ \\
\hline Other (American-Hispanic) & $1(1.7)$ & $0(0)$ \\
\hline \multicolumn{3}{|l|}{ Marital status, n(\%) } \\
\hline Single/never married & $60(100)$ & $0(0)$ \\
\hline Married & $0(0)$ & $41(68.3)$ \\
\hline Divorced & $0(0)$ & $14(23.3)$ \\
\hline Widowed & $0(0)$ & $5(8.3)$ \\
\hline \multicolumn{3}{|l|}{ Highest level of education completed, n(\%) } \\
\hline Less than 7 th grade & $5(8.3)$ & $0(0)$ \\
\hline Some junior high school & $1(1.7)$ & $0(0)$ \\
\hline Some high school & $9(15.0)$ & $0(0)$ \\
\hline $\begin{array}{l}\text { High school graduate (including certificate of } \\
\text { attendance/special education certificate) }\end{array}$ & $41(68.3)$ & $5(8.3)$ \\
\hline Some college & $4(6.7)$ & $16(26.7)$ \\
\hline College degree & $0(0)$ & $22(36.7)$ \\
\hline Graduate/master's degree & $0(0)$ & $10(16.7)$ \\
\hline Doctoral degree & $0(0)$ & $7(11.7)$ \\
\hline
\end{tabular}


Table 4-1. (continued)

\begin{tabular}{lcc}
\hline Characteristics & $\begin{array}{c}\text { Adult with DS } \\
(\mathbf{n = 6 0 )}\end{array}$ & $\begin{array}{c}\text { Caregiver } \\
(\mathbf{n = 6 0})\end{array}$ \\
\hline Employment status, $\mathrm{n}(\%)$ & & \\
$\quad$ Retired & $0(0)$ & $19(31.7)$ \\
Works full-time (30 hours or more per week) & $2(3.3)$ & $26(43.3)$ \\
Works part-time (29 hours or less per week) & $22(36.7)$ & $9(15.0)$ \\
Stay-at-home parent & $0(0)$ & $5(8.3)$ \\
Unemployed & $27(45.0)$ & $1(1.7)$ \\
Volunteers & $9(15.0)$ & $0(0)$ \\
\hline
\end{tabular}

Note. Data collected from caregivers of adults with DS

Table 4-2. Daily activities of adults with Down syndrome $(n=60)$

\begin{tabular}{lr}
\hline Activity & $\mathbf{n}(\mathbf{\%})$ \\
\hline Daily activities of adults with DS (selected all that applied) & \\
Works in paid position full-time (30 hours or more per week) & $2(3.3)$ \\
Works in paid position part-time (29 hours or less per week) & $22(36.7)$ \\
Volunteers & $20(33.3)$ \\
Attends vocational training program & $5(8.3)$ \\
Attends day program & $20(33.3)$ \\
Attends high-school & $4(6.7)$ \\
No routine activity & $6(10.0)$ \\
& \\
Daily activities adults with DS spends most time doing & \\
Works in paid position full-time (30 hours or more per week) & $0(0)$ \\
Works in paid position part-time (29 hours or less per week) & $16(26.7)$ \\
Volunteers & $7(11.7)$ \\
Attends vocational training program & $2(3.3)$ \\
Attends day program & $12(20.0)$ \\
Attends high-school & $3(5.0)$ \\
No routine activity & $5(8.3)$ \\
Missing/none of the above & $15(25.0)$ \\
& \\
Work situation/support of employed adults with DS & \\
Independent & $6(10.0)$ \\
Supported & $3(5.0)$ \\
Sheltered Workshop & $5(8.3)$ \\
\hline
\end{tabular}

Note. Data collected from caregivers of adults with DS 
Table 4-3. Living arrangements of adults with Down syndrome and their relationships with caregivers $(n=60)$

\begin{tabular}{lr}
\hline Variable & $\mathbf{n ( \% )}$ \\
\hline Living arrangements of adults with DS & \\
With friend(s) independently & $1(1.7)$ \\
With parent & $53(88.3)$ \\
With sibling & $4(6.7)$ \\
With other relative & $1(1.7)$ \\
In a supervised or staffed environment & $1(1.7)$ \\
& \\
Adults with DS living with respondent caregiver & $58(96.7)$ \\
& \\
Relationship of caregiver to adult with DS & \\
Parent & $55(91.7)$ \\
Sibling & $3(5.0)$ \\
Other family relationship (aunt) & $1(1.7)$ \\
Friend & $1(1.7)$ \\
Frequency of face-to-face contact between adult with DS and & \\
caregiver & \\
Daily & \\
Once or twice per week & $59(98.3)$ \\
\hline
\end{tabular}

Note. Data collected from caregivers of adults with DS 


\section{Specific Aim One}

Specific aim one was to measure the HRQOL of adults with DS. The research question to achieve this specific aim is:

Research Question 1 What is the HRQOL of adults with DS as reported by:

a) adults with DS?

b) caregivers of adults with DS?

The HRQOL of adults with DS was measured using the QualityMetric Short Form-12v2 Health Survey (SF-12v2), which was administered verbally to adults with DS (question 1a) and in written form to the caregivers (question 1b). The data were entered into the QualityMetric Health Outcomes ${ }^{\mathrm{TM}}$ Scoring Software 4.5 for scoring and then aggregated using the International Business Machine Corporation Statistical Package for Social Sciences (SPSS) version 20. For all scales and summary scores, a higher value indicates better health (Saris-Baglama et al., 2011).

During data collection, the investigator estimated that some adults with DS had difficulty comprehending some questions on the SF-12v2 and providing a consistent response. This was demonstrated by the adult with DS not selecting one of the standardized answer choices after being offered the choices two at a time as discussed previously or by providing inconsistent responses when asked the same question a second time or in a different way. Because of this unanticipated finding, a variable was created to permit the investigator to subjectively rate the number of questions that the adult with DS answered appropriately and consistently with confirmation (Table 4-4). This resulted in a subgroup of 44 adults with DS (Subgroup A) who demonstrated adequate comprehension of more than half of the questions on the SF-12v2: 17 adults with DS who demonstrated understanding of 7-9 questions, and 27 adults who demonstrated understanding of 10-12 questions. There were 16 adults with DS who demonstrated adequate comprehension of half or less than half of the items on the SF-12v2 (Subgroup B). The means of the self-reported SF-12v2 norm-based scores and component summary scores for both Subgroup A $(n=44)$ and Subgroup B $(n=16)$ were compared using independent samples $t$ tests. Levene's test confirmed that the assumption of equal

Table 4-4. Investigator's confidence rating of Short Form-12v2 responses by adults with Down syndrome $(n=60)$

\begin{tabular}{lc}
\hline $\begin{array}{l}\text { Number of questions with confirmed } \\
\text { understanding on the SF-12v2 }\end{array}$ & $\mathbf{n ( \% )}$ \\
\hline 0 of 12 & $4(6.7)$ \\
$1-3$ of 12 & $4(6.7)$ \\
$4-6$ of 12 & $8(13.3)$ \\
$7-9$ of 12 & $17(28.3)$ \\
$10-12$ of 12 & $27(45.0)$ \\
\hline
\end{tabular}


variance between the two groups was not violated. No significant differences were identified. Means of the caregiver-reported scores for the both subgroups were also analyzed. There was no violation of the assumption of equal variance, and no significant differences were identified between these subgroups of caregivers.

Psychometric properties for the SF-12v2 for the 60 participating adults with DS include convergent validity at .83 and discriminant validity at 1.00 calculated using the QualityMetric Health Outcomes ${ }^{\mathrm{TM}}$ Scoring Software 4.5. Both convergent validity and discriminant validity were 1.00 for the SF-12v2 surveys completed by the caregivers of adults with DS. The convergent validity for adults with DS in Subgroup A $(n=44)$ and Subgroup B ( $\mathrm{n}=16)$ was also .83, and the discriminant validity for both subgroups was 1.00. The PCS and MCS scores of the caregivers of both subgroups had convergent validity and discriminant validity of 1.00. Reliability and validity have been reported as adequate in a large, national probability study that included self-administered and face-toface interviews with adults ( $\mathrm{N}=20,699$; Cheak-Zamora, Wyrwich, \& McBride, 2009). However, the calculation of convergent and discriminant validity was recommended for this study, because calculation of a Cronbach's alpha is not a feature of the QualityMetric Health Outcomes ${ }^{\mathrm{TM}}$ Scoring Software 4.5 for the SF-12v2 (P. Bartley, personal communication, March 27, 2014; Saris-Baglama et al., 2011).

\section{Research question 1a findings}

Measures of central tendency and variance of the norm-based scores and component summary scores of the SF-12v2 are reported in Table 4-5. Per self-report by the entire sample of adults with DS, the norm-based scores were above average for all

Table 4-5. Health-related quality of life scores of adults with Down syndrome obtained via self-report $(n=60)$ and caregiver-report $(n=60)$

\begin{tabular}{lccc}
\hline $\begin{array}{l}\text { Scales and component scores from } \\
\text { SF-12v2 }\end{array}$ & $\begin{array}{c}\text { Self-report } \\
\text { M(SD) }\end{array}$ & $\begin{array}{c}\text { Caregiver- } \\
\text { report } \\
\text { M(SD) }\end{array}$ & $\begin{array}{c}\text { Paired } \boldsymbol{t} \\
\text { statistic }\end{array}$ \\
\hline Physical functioning norm-based score & $52.78(6.72)$ & $52.12(8.82)$ & .574 \\
Role physical norm-based score & $54.76(4.21)$ & $52.37(7.21)$ & $2.428^{*}$ \\
Bodily pain norm-based score & $50.62(12.20)$ & $53.63(6.73)$ & -1.646 \\
General health norm-based score & $56.97(8.53)$ & $56.16(6.93)$ & .602 \\
Vitality norm-based score & $57.77(8.25)$ & $57.16(7.22)$ & .497 \\
Social functioning norm-based score & $55.41(2.58)$ & $54.64(4.87)$ & 1.150 \\
Role emotional norm-based score & $51.37(5.69)$ & $51.95(7.01)$ & -.544 \\
Mental health norm-based score & $54.08(7.40)$ & $54.91(6.69)$ & -.655 \\
Physical component summary score & $53.80(5.98)$ & $53.13(7.82)$ & .542 \\
Mental component summary score & $54.46(5.95)$ & $54.93(6.40)$ & -.439 \\
\hline
\end{tabular}

$* \mathrm{p}<.05$ 
scales. The physical component summary (PCS) score via self-report was 53.80 and the mental component summary (MCS) score was 54.46, both higher than the U.S. average of 50 (Saris-Baglama et al., 2011).

The data for specific aim one were also analyzed separately for the adults with DS in Subgroup A $(n=44)$ who reliably answered 7 or more questions on the SF-12v2 (Table 4-6). All norm-based scores and component summary scores for Subgroup A were also above average.

\section{Research question $1 b$ findings}

Like the norm-based scores from self-report, all norm-based scores from caregiver-report were above average (Table 4-5). Caregiver-reported PCS and caregiverreported MCS were again higher than the U.S. average of 50.

All caregiver-reported norm-based scores in Subgroup A were also above average (Table 4-6). The caregiver-reported PCS and MCS for Subgroup A were above average as well.

\section{Specific Aim Two}

Specific aim two was to compare the HRQOL of adults with DS from data obtained via self-report to that obtained via caregiver-report. The research question to address this specific aim is:

Table 4-6. Health-related quality of life scores of adults with Down syndrome with interviewer's confidence levels 7-12 obtained via self-report $(n=44)$ and caregiver-report $(n=44)$

\begin{tabular}{llcr}
\hline $\begin{array}{l}\text { Scales and component scores from } \\
\text { SF-12v2 }\end{array}$ & $\begin{array}{c}\text { Self-report } \\
\text { M(SD) }\end{array}$ & $\begin{array}{c}\text { Caregiver- } \\
\text { report } \\
\text { M(SD) }\end{array}$ & $\begin{array}{c}\text { Paired } \boldsymbol{t} \\
\text { statistic }\end{array}$ \\
\hline Physical functioning norm-based score & $53.30(6.26)$ & $51.69(9.26)$ & 1.102 \\
Role physical norm-based score & $55.07(4.31)$ & $52.86(6.99)$ & 1.943 \\
Bodily pain norm-based score & $50.04(13.01)$ & $53.28(7.16)$ & -1.402 \\
General health norm-based score & $56.94(8.67)$ & $55.97(6.88)$ & .589 \\
Vitality norm-based score & $57.85(8.14)$ & $57.02(6.95)$ & .645 \\
Social functioning norm-based score & $55.34(2.69)$ & $54.92(3.78)$ & .628 \\
Role emotional norm-based score & $51.31(5.15)$ & $52.48(6.36)$ & -1.040 \\
Mental health norm-based score & $54.09(6.96)$ & $54.47(6.34)$ & -.277 \\
Physical component summary score & $53.96(6.00)$ & $52.95(8.34)$ & .655 \\
Mental component summary score & $54.34(5.57)$ & $55.07(6.14)$ & -.651 \\
\hline
\end{tabular}


Research Question 2 What are the associations between the HRQOL of adults with DS as reported by adults with DS and their caregivers?

The measures of central tendency and variance described for specific aim one were used to evaluate the associations between the HRQOL of all adults with DS obtained via self-report to that obtained via caregiver-report. Using SPSS, pairedsamples $t$ tests were conducted to analyze differences, and Pearson product-moment correlations were conducted to analyze similarities. The significant differences (Table 4-5) occurred between the means of self-reported and caregiver-reported role physical norm-based scores $(t=2.428, \mathrm{p}=.018)$. The only significant association was found with the physical functioning norm-based score $(\mathrm{r}=.373, \mathrm{p}=.003)$. There were no additional associations in the evaluation of the scores.

The same analysis of associations was conducted on Subgroup A ( $\mathrm{n}=44$; Table 4-6). There were no significant differences between the means of the self-report and caregiver-report, and the only significant association was between self-reported and caregiver-reported vitality norm-based score $(\mathrm{r}=.358, \mathrm{p}=.017)$.

\section{Specific Aim Three}

The third specific aim was to investigate the health of adults with DS from the perspective of the adults with DS and from that of the caregivers. The research question to guide this specific aim is:

Research Question 3 What are the associations between health problems, health risks, and health-care utilization of adults with DS as reported by adults with DS and their caregivers?

The semi-structured interviews of adults with DS were focused on the health problems, health risks, and health-care utilization of adults with DS. The data from each interview were quantitized as described previously. The quantitized data were then entered into SPSS to permit analysis concordant with that obtained from the caregivers using the Health Questionnaire to gain their insight into the health problems, health risks, and health-care utilization of adults with DS. Frequencies and measures of central tendency and variance were evaluated.

One notable limitation of this specific aim is that questions were posed differently to the adults with DS to avoid yes/no bias and to ascertain their thoughts and understanding about their health. Because of this, it was challenging to match this information completely with that obtained via caregiver-report from a questionnaire, which lends to greater certainty of positive or negative responses. Therefore, data were then recoded to improve the reliability of comparative statistical analysis. Health problem and health risk data from self-report of adults with DS were coded into the dichotomous responses of "yes" and of "no", which combined "no" and "not mentioned". The same two options were already present in the data regarding health problems and 
health risks from the caregivers, and missing data were not included in analysis. Data from health-care utilization were recoded to dichotomous responses to permit analysis. Therefore, responses from adults with DS were recoded into "no" combining "never" and "not mentioned"; and "yes" combining "in the last month", "in the last year", "in the last two years", "more than two years ago", and "mentioned without time frame". This obviously diluted the precision of these data, but the awareness affords an overview on which to build future research.

To analyze differences between self- and caregiver-reported health problems, health risks, and health-care utilization of adults with DS, McNemar tests were conducted. To analyze differences between the self- and caregiver-reported sums of health problems and sums of health risks, paired-samples $t$ tests were conducted. To analyze associations between self- and caregiver-reported health problems, health risks, and health-care utilization, point-biserial correlations were conducted, and for sums of health problems and of health risks, Pearson product-moment correlations were calculated.

Self-reported frequencies and percentages of the health problems of adults with DS are reported in Table 4-7. Most of the adults with DS specified having problems with aches and pains and other health problems not listed on the Health Questionnaire. The most common locations of aches and pains included the back, knees, legs, and feet. The most common other health problems self-reported were nausea/vomiting, stomach ache, sore throat, ear problems, and anger. Other self-reported health problems were anxiety/stress, daydreams, and psychosis. Out of the 30 women with DS, $43.3 \%$ stated that they had irregular, painful, or heavy menstrual cycles. The self-reported health problems were totaled for each adult with DS, and a mean (4.93) and standard deviation (2.62) were calculated.

Caregiver-reported frequencies and percentages of the health problems of adults with DS are presented in Table 4-7. Most of the caregivers indicated that adults with DS have thyroid problems and heart problems now or in the past. The next most common problems were allergies to environmental irritants, foods, animals, and/or medications; problems with weight; and aches and pains. The more common locations of aches and pains reported by the caregivers were the knees, legs, feet, and back. Only $31.7 \%$ of the caregivers stated the adults with DS had other health problems not listed, and the most common of these were skin problems, including psoriasis, rashes, extreme dryness, and eczema; ear problems; a history of abdominal hernia repair; gout; and joint dysplasia. Caregivers of the 30 women with DS stated that $46.7 \%$ had irregular, painful, or heavy menstrual cycles. The caregiver-reported health problems were totaled for each adult with DS, and a mean (6.77) and standard deviation (4.03) were calculated.

Significant differences between self- and caregiver-reported health problems are also presented in Table 4-7. The health problems that differed the most with a McNemar p-value of .000 were other health problems, problems hearing, problems remembering, thyroid problems, and heart problems now or in the past. A significantly higher number 
Table 4-7. Health problems of adults with Down syndrome obtained via self-report $(\mathrm{n}=60)$ and caregiver-report $(n=60)$

\begin{tabular}{|c|c|c|c|c|}
\hline Health problem & $\begin{array}{c}\text { Self-report } \\
\text { n(\%) }\end{array}$ & $\begin{array}{c}\text { Caregiver- } \\
\text { report n(\%) }\end{array}$ & $\begin{array}{c}\text { McNemar } \\
\text { test p-value }\end{array}$ & $\begin{array}{l}\text { Correlation } \\
\text { coefficient }^{\text {ab }}\end{array}$ \\
\hline Other health problems & $47(78.3)$ & $19(31.7)$ & $.000 * * *$ & .078 \\
\hline Aches and pains & $38(63.3)$ & $25(41.7)$ & $.007 * *$ & $.362 * *$ \\
\hline Problems seeing & $22(36.7)$ & $24(40.0)$ & .855 & -.056 \\
\hline Allergies & $22(36.7)$ & $29(48.3)$ & .230 & .164 \\
\hline Problems with weight & $19(31.7)$ & $26(43.3)$ & .189 & $.272 *$ \\
\hline Feeling sad or blue & $17(28.3)$ & $6(10.0)$ & $.013 *$ & .160 \\
\hline Thyroid problems & $17(28.3)$ & $32(53.3)$ & $.000 * * *$ & $.514 * * *$ \\
\hline Problems with appetite & $15(25.0)$ & $4(6.7)$ & $.007 * *$ & .154 \\
\hline Problems with teeth or gums & $13(21.7)$ & $18(30.0)$ & .332 & $.269 *$ \\
\hline Headaches & $12(20.0)$ & $12(20.0)$ & 1.00 & $.479 * * *$ \\
\hline Heart problems now or in the past & $7(11.7)$ & $32(53.3)$ & $.000 * * *$ & .236 \\
\hline Problems with coughing & $7(11.7)$ & $11(18.3)$ & .454 & -.038 \\
\hline Heartburn & $6(10.0)$ & $10(16.7)$ & .289 & $.447 * * *$ \\
\hline Problems with constipation & $6(10.0)$ & $14(23.3)$ & .077 & .079 \\
\hline Trouble walking & $5(8.3)$ & $7(11.7)$ & .727 & $.266^{*}$ \\
\hline Dizziness or weak spells & $4(6.7)$ & $7(11.7)$ & .508 & .111 \\
\hline Problems with breathing while asleep & $4(6.7)$ & $15(25.0)$ & $.003 * *$ & $.309 *$ \\
\hline Arthritis & $3(5.0)$ & $11(18.3)$ & $.008 * *$ & $.484 * * *$ \\
\hline Problems hearing & $3(5.0)$ & $18(30.0)$ & $.000 * * *$ & .184 \\
\hline Problems reading or identifying small objects & $3(5.0)$ & $13(21.7)$ & $.021 *$ & -.121 \\
\hline Problems remembering & $3(5.0)$ & $18(30.0)$ & $.000 * * *$ & .184 \\
\hline High blood pressure & $2(3.3)$ & $2(3.3)$ & 1.000 & $.483 * * *$ \\
\hline Circulatory problems/blood clots & $2(3.3)$ & $6(10.0)$ & .219 & .248 \\
\hline Go to the bathroom at night & $1(1.7)$ & $11(18.3)$ & $.006 * *$ & -.062 \\
\hline Colds often & $1(1.7)$ & $5(8.3)$ & .219 & -.039 \\
\hline Shortness of breath & $1(1.7)$ & $3(5.0)$ & .625 & -.030 \\
\hline Asthma & $1(1.7)$ & $2(3.3)$ & 1.000 & $.701 * * *$ \\
\hline Trouble getting out of a chair & $0(0)$ & $2(3.3)$ & .500 & $\dagger$ \\
\hline
\end{tabular}


Table 4-7. (continued)

\begin{tabular}{|c|c|c|c|c|}
\hline Health problem & $\begin{array}{c}\text { Self-report } \\
\text { n(\%) }\end{array}$ & $\begin{array}{c}\text { Caregiver- } \\
\text { report n(\%) }\end{array}$ & $\begin{array}{c}\text { McNemar } \\
\text { test p-value }^{\text {a }}\end{array}$ & $\begin{array}{l}\text { Correlation } \\
\text { coefficient }^{\text {ab }}\end{array}$ \\
\hline Seizures & $0(0)$ & $1(1.7)$ & 1.000 & $\dagger$ \\
\hline Diabetes & $0(0)$ & $3(5.0)$ & .250 & $\dagger$ \\
\hline Cancer & $0(0)$ & $0(0)$ & $\dagger$ & $\dagger$ \\
\hline $\begin{array}{l}\text { Problems with monthly period or menstrual } \\
\text { cycle (women only, } n=30 \text { ) }\end{array}$ & $13(43.3)$ & $14(46.7)$ & 1.000 & $.396 *$ \\
\hline Menopause (women only, $n=30$ ) & $2(6.7)$ & $5(16.7)$ & .250 & $.598 * * *$ \\
\hline Prostate problems (men only, $\mathrm{n}=30$ ) & $0(0)$ & $1(3.3)$ & 1.000 & $\dagger$ \\
\hline
\end{tabular}

Note. "yes" responses reported

${ }^{a}$ Responses analyzed were "no" ("no" and "not mentioned") and "yes"; missing data not included

${ }^{b}$ Point-biserial correlation coefficient

$\uparrow$ unable to calculate

$* \mathrm{p}<.05 ; * * \mathrm{p}<.01 ; * * * \mathrm{p}<.001$ 
of adults with DS reported having problems with appetite and aches and pains than the caregivers. A significantly higher number of caregivers reported adults with DS having trouble breathing while asleep and going to the bathroom at night. The sum of health problems had a significant difference between self- and caregiver-report with a $t$ statistic of $-3.242(\mathrm{p}=.002)$.

Self-reported frequencies and percentages of the health risks of adults with DS are displayed in Table 4-8. The health risks of adults with DS per self-report include consumption of alcohol and smoking cigarettes. Most of those who consumed alcohol stated that did so rarely/occasionally ( $38.3 \%$ of the sample), $13.3 \%$ stated they drank only once/a few times in their lives, and $6.7 \%$ stated they had one or more drinks per day. The two individuals who stated that they smoked cigarettes indicated that they had smoked once or perhaps a few times ever. The self-reported health risks were totaled for each adult with DS, and a mean (.63) and standard deviation (.52) were calculated.

Caregiver-reported frequencies and percentages of the health risks of adults with DS are also reported in Table 4-8 and include consumption of alcohol. Caregivers reported frequency of alcohol consumption by adults with DS as rarely/occasionally $(23.3 \%)$, one or more drinks per week $(10 \%)$, and one or more drinks per day $(1.7 \%)$. The caregiver-reported health risks were totaled for each adult with DS, and a mean (.38) and standard deviation (.49) were calculated.

Differences between self- and caregiver-reported health risks (Table 4-8) were drinking alcohol $(\mathrm{p}=.011)$ and the sum of health risks $(t=3.227, \mathrm{p}=.002)$. Associations

Table 4-8. Health risks of adults with Down syndrome obtained via self-report $(n=60)$ and caregiver-report $(n=60)$

\begin{tabular}{lrccc}
\hline Health risk & $\begin{array}{c}\text { Self-report } \\
\mathbf{n}(\%)\end{array}$ & $\begin{array}{c}\text { Caregiver- } \\
\text { report n(\%) }\end{array}$ & $\begin{array}{c}\text { McNemar } \\
\text { test p-value }^{\mathbf{a}}\end{array}$ & $\begin{array}{c}\text { Correlation }^{\text {poefficient }} \\
\text { cob }^{\mathbf{a b}}\end{array}$ \\
\hline Drinks alcohol & $36(60.0)$ & $23(38.3)$ & $.011^{*}$ & $.294^{*}$ \\
Smokes & $2(3.3)$ & $0(0)$ & .500 & $\dagger$ \\
Uses birth control & $2(3.3)$ & $5(8.3)$ & .250 & $.616^{* * *}$ \\
Exposed to second- & $0(0)$ & $0(0)$ & $\dagger$ & $\dagger$ \\
$\quad$ hand smoke & $0(0)$ & $0(0)$ & $\dagger$ & $\dagger$ \\
Uses street drugs & $0(0)$ & $0(0)$ & $\dagger$ & $\dagger$ \\
Is sexually active & & & $\dagger$ \\
\hline
\end{tabular}

Note. "yes" responses reported

aResponses analyzed were "no" ("no" and "not mentioned") and "yes"; missing data not included

${ }^{\mathrm{b}}$ Point-biserial correlation coefficient

$\dagger$ unable to calculate

${ }^{*} \mathrm{p}<.05 ; * * * \mathrm{p}<.001$ 
between self- and caregiver-reported health risks were drinking alcohol $(r=.294 ; p=.023)$ and sum of health risks $(r=.295 ; \mathrm{p}=.022)$.

Self-reported frequencies and percentages of the health-care utilization of adults with DS (Table 4-9), indicated that, although 55\% of adults with DS stated that they did have a primary care provider and many remembered provider visits, $78.3 \%$ did not discuss the most recent visit to their primary care providers in the interviews (mentioned without timeframe). Less common health-care visits and tests were generally not mentioned at all during the interviews. One adult with DS mentioned health insurance (Medicaid) during the interview. None of the adults with DS claimed to have an obstacle to seeking or receiving health care, but $63.3 \%$ indicated some sort of resistance to or dislike about health care. Of these resistances/dislikes, most stemmed from fear of needle sticks, fear of the dentist \&/or drill, unfamiliarity with the provider, embarrassment, and reliance on others to make and get to appointments. This resistance to seeking/receiving health care was a question not asked of the caregivers; rather this information emerged during the interviews with the adult with DS.

Caregiver-reported frequencies and percentages of the health-care utilization of adults with DS (Table 4-9) indicate that within the last year, most adults with DS visited a health-care provider (58.3\%), a dentist (61.7\%), and an eye specialist (56.7\%), and $51.7 \%$ received an influenza vaccination. The most recent cervical spine $\mathrm{x}$-ray to assess for atlantoaxial instability for most adults with DS was more than two years ago $(66.7 \%)$. The caregivers noted that most adults with DS have a primary care provider $(96.7 \%)$, are insured by Medicare (51.7\%), and do not have an obstacle to seeking or receiving health care. According to caregivers, most adults with DS had two insurance providers, and some had three. One caregiver reported that the adult with DS was in a five-month period of not being covered by health insurance at the time of the data collection session. Her private policy had been canceled, and she was awaiting Medicare coverage to begin. No caregiver reported an adult with DS having an obstacle to receiving or seeking health care.

Significant differences between self- and caregiver-reported health-care utilization (Table 4-10) include the adult with DS having a primary care provider $(\mathrm{p}=.000)$. Differences in self- and caregiver-report for insurance coverage of adults with DS were significant for private insurance, Medicaid, and Medicare (all $p=.000$ ). There were no significant differences between adults with DS and their caregivers in their report of the last visit to a health-care provider and eye specialist and the last mammogram for women with DS. The only significant association between the healthutilization of adults with DS obtained via self-report and that obtained via caregiverreport was the last visit to an eye specialist $(\mathrm{r}=.487 ; \mathrm{p}=.000)$. 
Table 4-9. Health-care utilization of adults with Down syndrome obtained via self-report $(n=60)$ and caregiver-report $(n=60)$

\begin{tabular}{|c|c|c|}
\hline Health-care utilization & $\begin{array}{c}\text { Self-report } \\
n(\%)\end{array}$ & $\begin{array}{l}\text { Caregiver- } \\
\text { report } n(\%)\end{array}$ \\
\hline \multicolumn{3}{|c|}{$\begin{array}{l}\text { Last visit to a health-care provider (doctor, nurse } \\
\text { practitioner or physician assistant) }\end{array}$} \\
\hline In the last month & $5(8.3)$ & $22(36.7)$ \\
\hline In the last year & $7(11.7)$ & $35(58.3)$ \\
\hline In the last two years & $0(0)$ & $1(1.7)$ \\
\hline More than two years ago & $0(0)$ & $2(3.3)$ \\
\hline Never & $0(0)$ & $0(0)$ \\
\hline Mentioned without time frame & $47(78.3)$ & $0(0)$ \\
\hline Not mentioned/missing & $1(1.7)$ & $0(0)$ \\
\hline \multicolumn{3}{|l|}{ Last visit to a dentist } \\
\hline In the last month & $1(1.7)$ & $14(23.3)$ \\
\hline In the last year & $4(6.7)$ & $37(61.7)$ \\
\hline In the last two years & $0(0)$ & $5(8.3)$ \\
\hline More than two years ago & $0(0)$ & $4(6.7)$ \\
\hline Never & $4(6.7)$ & $0(0)$ \\
\hline Mentioned without time frame & $46(76.7)$ & $0(0)$ \\
\hline Not mentioned/missing & $5(8.3)$ & $0(0)$ \\
\hline \multicolumn{3}{|l|}{ Last visit to an eye specialist } \\
\hline In the last month & $0(0)$ & $3(5.0)$ \\
\hline In the last year & $1(1.7)$ & $34(56.7)$ \\
\hline In the last two years & $0(0)$ & $15(25.0)$ \\
\hline More than two years ago & $1(1.7)$ & $7(11.7)$ \\
\hline Never & $2(3.3)$ & $1(1.7)$ \\
\hline Mentioned without time frame & $54(90.0)$ & $0(0)$ \\
\hline Not mentioned/missing & $2(3.3)$ & $0(0)$ \\
\hline \multicolumn{3}{|c|}{$\begin{array}{l}\text { Last visit to an emergency department or urgent care } \\
\text { center }\end{array}$} \\
\hline In the last month & $0(0)$ & $3(5.0)$ \\
\hline In the last year & $0(0)$ & $11(18.3)$ \\
\hline In the last two years & $0(0)$ & $7(11.7)$ \\
\hline More than two years ago & $1(1.7)$ & $23(38.3)$ \\
\hline Never & $0(0)$ & $14(23.3)$ \\
\hline Mentioned without time frame & $5(8.3)$ & $0(0)$ \\
\hline Not mentioned/missing & $54(90.0)$ & $2(3.3)$ \\
\hline
\end{tabular}


Table 4-9. (continued)

\begin{tabular}{|c|c|c|}
\hline Health-care utilization & $\begin{array}{c}\text { Self-report } \\
\text { n(\%) }\end{array}$ & $\begin{array}{l}\text { Caregiver- } \\
\text { report n(\%) }\end{array}$ \\
\hline \multicolumn{3}{|l|}{ Last time hearing was checked } \\
\hline In the last month & $0(0)$ & $9(15.0)$ \\
\hline In the last year & $1(1.7)$ & $17(28.3)$ \\
\hline In the last two years & $0(0)$ & $13(21.7)$ \\
\hline More than two years ago & $1(1.7)$ & $17(28.3)$ \\
\hline Never & $7(11.7)$ & $2(3.3)$ \\
\hline Mentioned without time frame & $35(58.3)$ & $0(0)$ \\
\hline Not mentioned/missing & $16(26.7)$ & $2(3.3)$ \\
\hline \multicolumn{3}{|c|}{$\begin{array}{l}\text { Last time thyroid was checked (physical exam or } \\
\text { blood levels of thyroid hormones) }\end{array}$} \\
\hline In the last month & $1(1.7)$ & $12(20.0)$ \\
\hline In the last year & $1(1.7)$ & $27(45.0)$ \\
\hline In the last two years & $0(0)$ & $5(8.3)$ \\
\hline More than two years ago & $0(0)$ & $11(18.3)$ \\
\hline Never & $0(0)$ & $4(6.7)$ \\
\hline Mentioned without time frame & $6(10.0)$ & $0(0)$ \\
\hline Not mentioned/missing & $52(86.7)$ & $1(1.7)$ \\
\hline \multicolumn{3}{|l|}{ Last neck x-ray } \\
\hline In the last month & $0(0)$ & $1(1.7)$ \\
\hline In the last year & $0(0)$ & $4(6.7)$ \\
\hline In the last two years & $0(0)$ & $7(11.7)$ \\
\hline More than two years ago & $0(0)$ & $40(66.7)$ \\
\hline Never & $0(0)$ & $7(11.7)$ \\
\hline Mentioned without time frame & $0(0)$ & $0(0)$ \\
\hline Not mentioned/missing & $60(100.0)$ & $1(1.7)$ \\
\hline \multicolumn{3}{|l|}{ Last tuberculosis (TB) test } \\
\hline In the last month & $1(1.7)$ & $4(6.7)$ \\
\hline In the last year & $0(0)$ & $5(8.3)$ \\
\hline In the last two years & $0(0)$ & $12(20.0)$ \\
\hline More than two years ago & $0(0)$ & $17(28.3)$ \\
\hline Never & $0(0)$ & $16(26.7)$ \\
\hline Mentioned without time frame & $0(0)$ & $0(0)$ \\
\hline Not mentioned/missing & $59(98.3)$ & $6(10.0)$ \\
\hline
\end{tabular}


Table 4-9. (continued)

\begin{tabular}{|c|c|c|}
\hline Health-care utilization & $\begin{array}{c}\text { Self-report } \\
\text { n(\%) }\end{array}$ & $\begin{array}{l}\text { Caregiver- } \\
\text { report } n(\%)\end{array}$ \\
\hline \multicolumn{3}{|l|}{ Last flu shot } \\
\hline In the last month & $0(0)$ & $4(6.7)$ \\
\hline In the last year & $0(0)$ & $31(51.7)$ \\
\hline In the last two years & $0(0)$ & $6(10.0)$ \\
\hline More than two years ago & $0(0)$ & $4(6.7)$ \\
\hline Never & $0(0)$ & $15(25.0)$ \\
\hline Mentioned without time frame & $6(10.0)$ & $0(0)$ \\
\hline Not mentioned/missing & $54(90.0)$ & $0(0)$ \\
\hline \multicolumn{3}{|c|}{$\begin{array}{l}\text { Last female (internal exam or Papanicolaou) exam } \\
\text { (women only, } n=30 \text { ) }\end{array}$} \\
\hline In the last month & $0(0)$ & $0(0)$ \\
\hline In the last year & $0(0)$ & $5(16.7)$ \\
\hline In the last two years & $0(0)$ & $7(23.3)$ \\
\hline More than two years ago & $0(0)$ & $4(13.3)$ \\
\hline Never & $0(0)$ & $14(46.7)$ \\
\hline Mentioned without time frame & $1(3.3)$ & $0(0)$ \\
\hline Not mentioned/missing & $29(96.7)$ & $0(0)$ \\
\hline \multicolumn{3}{|c|}{$\begin{array}{l}\text { Last mammogram or breast ultrasound (women } \\
\text { only, } n=30 \text { ) }\end{array}$} \\
\hline In the last month & $0(0)$ & $1(3.3)$ \\
\hline In the last year & $0(0)$ & $1(3.3)$ \\
\hline In the last two years & $0(0)$ & $3(10.0)$ \\
\hline More than two years ago & $0(0)$ & $0(0)$ \\
\hline Never & $0(0)$ & $23(76.7)$ \\
\hline Mentioned without time frame & $0(0)$ & $0(0)$ \\
\hline Not mentioned/missing & $30(100.0)$ & $2(6.7)$ \\
\hline \multicolumn{3}{|c|}{ Last prostate exam (men only, $n=30$ ) } \\
\hline In the last month & $0(0)$ & $1(3.3)$ \\
\hline In the last year & $0(0)$ & $2(6.7)$ \\
\hline In the last two years & $0(0)$ & $4(13.3)$ \\
\hline More than two years ago & $0(0)$ & $4(13.3)$ \\
\hline Never & $0(0)$ & $16(53.3)$ \\
\hline Mentioned without time frame & $0(0)$ & $0(0)$ \\
\hline Not mentioned/missing & $30(100.0)$ & $3(10.0)$ \\
\hline
\end{tabular}


Table 4-9. (continued)

\begin{tabular}{lrr}
\hline Health-care utilization & $\begin{array}{r}\text { Self-report } \\
\mathbf{n}(\mathbf{\%})\end{array}$ & $\begin{array}{r}\text { Caregiver- } \\
\text { report n(\%) }\end{array}$ \\
\hline Last exam of the testicles (men only, $\mathrm{n}=30)$ & $0(0)$ & $0(0)$ \\
In the last month & $0(0)$ & $11(36.7)$ \\
In the last year & $0(0)$ & $7(23.3)$ \\
In the last two years & $0(0)$ & $7(23.3)$ \\
More than two years ago & $0(0)$ & $4(13.3)$ \\
Never & $0(0)$ & $0(0)$ \\
Mentioned without time frame & $30(100.0)$ & $1(3.3)$ \\
Not mentioned/missing & & \\
& & \\
Type of insurance (selected all that apply) & $0(0)$ & $29(48.3)$ \\
Private & $1(1.7)$ & $29(48.3)$ \\
Medicaid & $0(0)$ & $31(51.7)$ \\
Medicare & $0(0)$ & $1(1.7)$ \\
None & $0(0)$ & $8(13.3)$ \\
Other & $0(0)$ & $3(5.0)$ \\
TennCare & $0(0)$ & $2(3.3)$ \\
Tricare for Life & $0(0)$ & $1(1.7)$ \\
supplemental & $0(0)$ & $1(1.7)$ \\
Viva Medicare & $0(0)$ & $1(1.7)$ \\
unspecified & $59(98.3)$ & $1(1.7)$ \\
Not mentioned/missing & & \\
Has regular primary care provider (doctor, nurse & $33(55.0)$ & $58(96.7)$ \\
practitioner, or physician assistant) & & \\
& & \\
\hline
\end{tabular}


Table 4-10. Associations between health-care utilization of adults with Down syndrome obtained via self-report $(n=60)$ and caregiver-report $(n=60)$

\begin{tabular}{|c|c|c|c|c|}
\hline Health-care utilization & $\begin{array}{l}\text { Self-report } \\
\text { n(\%) }\end{array}$ & $\begin{array}{l}\text { Caregiver- } \\
\text { report } n(\%)\end{array}$ & $\begin{array}{l}\text { McNemar } \\
\text { test p-value }\end{array}$ & $\begin{array}{l}\text { Correlation } \\
\text { coefficient }^{\text {ab }}\end{array}$ \\
\hline Last visit to a health-care provider & $59(98.3)$ & $60(100)$ & 1.000 & $\dagger$ \\
\hline Last visit to a dentist & $51(85)$ & $60(100)$ & $.004 * *$ & $\dagger$ \\
\hline Last visit to an eye specialist & $56(93.3)$ & $59(98.3)$ & .250 & $.487 * * *$ \\
\hline Last visit to an emergency department or urgent care center & $6(10)$ & $44(73.3)$ & $.000 * * *$ & .059 \\
\hline Last time hearing was checked & $37(61.7)$ & $56(93.3)$ & $.000 * * *$ & .242 \\
\hline Last time thyroid was checked & $8(13.3)$ & $55(91.7)$ & $.000 * * *$ & .107 \\
\hline Last neck X-ray & $0(0)$ & $52(86.7)$ & $.000 * * *$ & $\dagger$ \\
\hline Last TB test & $1(1.7)$ & $38(63.3)$ & $.000 * * *$ & .089 \\
\hline Last flu shot & $6(10)$ & $45(75)$ & $.000 * * *$ & .064 \\
\hline Last female exam (women only, $\mathrm{n}=30$ ) & $1(3.3)$ & $16(53.3)$ & $.000 * * *$ & .174 \\
\hline Last mammogram or breast ultrasound (women only, $n=30$ ) & $0(0)$ & $5(16.7)$ & .063 & $\dagger$ \\
\hline Last exam of the testicles (men only, $\mathrm{n}=30$ ) & $0(0)$ & $25(83.3)$ & $.000 * * *$ & $\dagger$ \\
\hline Last prostate exam (men only, $\mathrm{n}=30$ ) & $0(0)$ & $11(36.7)$ & $.001 * *$ & $\dagger$ \\
\hline $\begin{array}{l}\text { Has regular primary care provider (doctor, nurse } \\
\text { practitioner, or physician assistant) }\end{array}$ & $33(55)$ & $58(96.7)$ & $.000 * * *$ & .205 \\
\hline \multicolumn{5}{|l|}{ Type of insurance } \\
\hline Private & $0(0)$ & $29(48.3)$ & $.000 * * *$ & $\dagger$ \\
\hline Medicaid & $1(1.7)$ & $29(48.3)$ & $.000 * * *$ & -1.290 \\
\hline Medicare & $0(0)$ & $31(51.7)$ & $.000 * * *$ & $\dagger$ \\
\hline None & $0(0)$ & $1(1.7)$ & 1.000 & $\dagger$ \\
\hline Other & $0(0)$ & $1(1.7)$ & $.008 * *$ & $\dagger$ \\
\hline
\end{tabular}

Note. "yes" responses reported

${ }^{a}$ Point-biserial correlation coefficient

bresponses analyzed are no ("never" and "not mentioned") and yes ("="in the last month", "in the last year", "in the last two years", "more than two years ago", and "mentioned without time frame"); missing data not included

$\dagger$ unable to calculate

$* * \mathrm{p}<.01 ; * * * \mathrm{p}<.001$ 


\section{Specific Aim Four}

The fourth specific aim was exploratory in nature and was to examine the association of the HRQOL of adults with DS to the health, adaptive behavior, and demographic characteristics of adults with DS. The following research question relates to this aim:

Research Question 4 What are the factors (health problems, health risks, healthcare utilization, adaptive behavior, and demographic characteristics) that are associated with the HRQOL of adults with DS as reported by:

a) adults with DS?

b) caregivers of adults with DS?

Due to the large number of variables in the factors of interest, the sample size was inadequate to achieve statistical power for multiple linear regression analysis. Therefore, the regression analysis was undertaken for exploratory purposes only to examine and identify potential factors that influence the HRQOL of adults with DS. To measure adaptive behavior of the adult participants with DS, the Vineland Adaptive Behavior Scales, Second Edition (Vineland-II) Parent/Caregiver Rating Form was completed by caregivers during the research meetings. Because the Vineland-II has three domain sums for seven-year-olds and above, the caregivers answered the survey regarding the communication, daily living skills, and socialization of the adults with DS (Table 4-11).

The next step was to analyze correlations of HRQOL scores with other data collected in the study. Four dependent variables, the PCS and MCS from the SF-12v2 via adults with DS and the PCS and MCS from the SF-12v2 via caregivers underwent correlational analysis using point-biserial correlation for dichotomous nominal independent variables (e.g., gender), Kruskal-Wallis tests with the dependent variable as a grouping variable with a range of 0-100 for non-dichotomous nominal independent variables (e.g., state of residence), Spearman's rho correlation with ordinal independent variables (e.g., level of education), and Pearson's product-moment correlation with interval independent variables (e.g., age of adults with DS). A predetermined cut point of correlations with a significance at or below .2 identified independent variables to be entered into the multiple linear regression models with the correlated dependent variable.

Table 4-12 presents demographic characteristics that had a correlation coefficient with a p-value at or below .2 with one or more of the four dependent variables. Table 4-13 presents correlations between the dependent variables and self-reported health problems, health risks, and health-care utilization of adults with DS with a p-value at or below the cut point of .2. Caregiver-reported health problems, health risks, and healthcare utilization correlated within the cut point with one or more dependent variables are presented in Table 4-14. No correlations with a p-value at or below the cut point were found between the Vineland-II scores and the self-reported PCS or MCS or caregiverreported PCS (Table 4-15). However, nine of the Vineland-II scores did correlate with 
Table 4-11. Adaptive behavior scores for adults with Down syndrome

\begin{tabular}{lr}
\hline Vineland-II scores, M(SD) & $\begin{array}{c}\text { Adult with DS } \\
\text { (n=60) }\end{array}$ \\
\hline Communication domain & $10.28(3.77)$ \\
Receptive communication subdomain v-scale score & $8.55(3.54)$ \\
Expressive communication subdomain v-scale score & $7.27(2.67)$ \\
Written communication subdomain v-scale score & $46.8(22.63)$ \\
Communication domain standard score & \\
& \\
Daily living skills domain & $8.45(2.61)$ \\
Personal daily living skills subdomain v-scale score & $8.62(3.13)$ \\
Domestic daily living skills subdomain v-scale score & $7.33(2.67)$ \\
Community daily living skills subdomain v-scale score & $57.97(14.04)$ \\
Daily living skills domain standard score & \\
Socialization domain & \\
Interpersonal relationships socialization subdomain v-scale & $9.3(3.09)$ \\
$\quad$ score & \\
Play and leisure socialization subdomain v-scale score & $9.97(1.98)$ \\
Coping socialization subdomain v-scale score & $10.83(3.44)$ \\
Socialization domain standard score & $64.13(17.06)$ \\
Adaptive behavior composite standard score & \\
\hline
\end{tabular}

Note. Data collected from caregivers of adults with DS 
Table 4-12. Correlation of the health-related quality of life of adults with Down syndrome via self- and caregiver-report to demographic characteristics of adults with Down syndrome and their caregivers

\begin{tabular}{|c|c|c|c|c|}
\hline Demographic characteristic & $\begin{array}{c}\text { SF-12v2 } \\
\text { PCS } \\
\text { self- } \\
\text { report }\end{array}$ & $\begin{array}{c}\text { SF-12v2 } \\
\text { MCS } \\
\text { self- } \\
\text { report }\end{array}$ & $\begin{array}{l}\text { SF-12v2 } \\
\text { PCS } \\
\text { caregiver } \\
\text {-report }\end{array}$ & $\begin{array}{c}\text { SF-12v2 } \\
\text { MCS } \\
\text { caregiver- } \\
\text { report }\end{array}$ \\
\hline State of residence $^{\mathrm{a}}$ & 14.15 & 19.64 & $32.15^{t}$ & $32.62^{t}$ \\
\hline Age of caregiver ${ }^{\mathrm{b}}$ & .076 & $-.273 * \$$ & .054 & -.166 \\
\hline Race of caregiver ${ }^{\mathrm{a}}$ & $35.10 *$ & $27.16^{+}$ & $33.9 * *$ & $38.51 * *$ \\
\hline Educational level of caregiver ${ }^{c}$ & .057 & -.034 & $.183^{\ddagger}$ & -.162 \\
\hline Employment status of caregiver ${ }^{\mathrm{a}}$ & 14.51 & 20.26 & 27.59 & 26.42 \\
\hline Relationship to adult with $\mathrm{DS}^{\mathrm{a}}$ & 20.91 & $26.13^{*}$ & 17.10 & 25.74 \\
\hline $\begin{array}{l}\text { Frequency of face to face } \\
\text { contact }^{\mathrm{c}}\end{array}$ & .049 & .124 & -.011 & $.207^{*}$ \\
\hline Age of adult with $\mathrm{DS}^{\mathrm{b}}$ & -.024 & $-.169^{*}$ & .049 & -.110 \\
\hline Gender of adult with $\mathrm{DS}^{\mathrm{d}}$ & .144 & $-.200^{\ddagger}$ & $.270 * \$$ & .005 \\
\hline Race of adult with $\mathrm{DS}^{\mathrm{a}}$ & $36.10 *$ & 20.87 & $35.52 * \$$ & $39.83 *$ \\
\hline $\begin{array}{l}\text { Educational level of adult with } \\
\mathrm{DS}^{\mathrm{c}}\end{array}$ & $.188^{\dagger}$ & -.077 & .128 & -.036 \\
\hline $\begin{array}{l}\text { Employment status of adult with } \\
\text { DS }^{\mathrm{a}}\end{array}$ & 24.60 & 19.40 & 20.29 & $27.74^{\ddagger}$ \\
\hline Daily activity: full-time job ${ }^{\mathrm{d}}$ & $-.209^{\ddagger}$ & .164 & $.168^{*}$ & $-.178^{\ddagger}$ \\
\hline Daily activity: part-time job ${ }^{\mathrm{d}}$ & .157 & -.043 & $.219^{\ddagger}$ & .138 \\
\hline Daily activity: day program ${ }^{\mathrm{d}}$ & .008 & -.035 & $-.181^{+}$ & .012 \\
\hline $\begin{array}{l}\text { Daily activity: high school } \\
\text { student }^{\mathrm{d}}\end{array}$ & $-.284 * *$ & $.245^{*}$ & -.022 & .017 \\
\hline Most frequent daily activity ${ }^{\mathrm{a}}$ & $26.10^{+}$ & 16.20 & 9.20 & 22.10 \\
\hline $\begin{array}{l}\text { Living arrangements of adult } \\
\text { with DS }\end{array}$ & 23.88 & $26.59^{\ddagger}$ & 21.06 & $33.76^{\ddagger}$ \\
\hline
\end{tabular}

Note. Demographic characteristics correlated with a p-value $\leq .2$ on one or more of the dependent variables are included in this table

${ }^{a}$ Kruskal-Wallis test $x^{2}$

${ }^{b}$ Pearson Product correlation coefficient

'Spearman's rho correlation coefficient

${ }^{\mathrm{d}}$ Point-biseral correlation coefficient

${ }^{\ddagger}$ p-values with cut-point of .2 to be included in multiple regression analysis

$* \mathrm{p}<.05$ 
Table 4-13. Correlation of the health-related quality of life of adults with Down syndrome via self- and caregiver-report to health problems, health risks, and health-care utilization per self-report

\begin{tabular}{|c|c|c|c|c|}
\hline $\begin{array}{l}\text { Self-reported health problem, } \\
\text { health risk, or health-care } \\
\text { utilization }\end{array}$ & $\begin{array}{c}\text { SF-12v2 } \\
\text { PCS } \\
\text { self- } \\
\text { report }\end{array}$ & $\begin{array}{c}\text { SF-12v2 } \\
\text { MCS } \\
\text { self- } \\
\text { report }\end{array}$ & $\begin{array}{c}\text { SF-12v2 } \\
\text { PCS } \\
\text { caregiver } \\
\text {-report }\end{array}$ & $\begin{array}{c}\text { SF-12v2 } \\
\text { MCS } \\
\text { caregiver } \\
\text {-report }\end{array}$ \\
\hline Trouble walking $^{\mathrm{a}}$ & -.108 & -.024 & $-.360 * *$ & -.043 \\
\hline Aches and pains ${ }^{\mathrm{a}}$ & -.239 & -.119 & $-.277 * \$$ & .007 \\
\hline Dizziness or weak spells ${ }^{a}$ & -.081 & -.012 & .059 & $-.216^{\ddagger}$ \\
\hline Headaches $^{\text {a }}$ & $-.183^{\ddagger}$ & $-.212^{\ddagger}$ & -.017 & .062 \\
\hline Problems hearing $^{\mathrm{a}}$ & $-.172^{\ddagger}$ & $-.190^{\ddagger}$ & -.106 & -.125 \\
\hline Problems seeing $^{\mathrm{a}}$ & .106 & -.140 & $.223^{\ddagger}$ & .004 \\
\hline $\begin{array}{l}\text { Problems reading or identifying } \\
\text { small objects }^{\mathrm{a}}\end{array}$ & $.210^{+}$ & -.057 & .165 & -.092 \\
\hline Feel sad or blue $\mathrm{a}^{\mathrm{a}}$ & .105 & $-.279 * \hbar$ & .043 & $-.264 * \$$ \\
\hline Problems remembering $^{\mathrm{a}}$ & .133 & -.021 & .053 & $-.256 * t$ \\
\hline Problems with appetite ${ }^{a}$ & -.108 & -.254 & $.251^{\dagger}$ & -.122 \\
\hline Problems with weight ${ }^{\mathrm{a}}$ & .135 & $-.176^{\ddagger}$ & -.158 & -.004 \\
\hline Thyroid problems ${ }^{\mathrm{a}}$ & .040 & -.040 & $-.176^{\ddagger}$ & -.055 \\
\hline Go to the bathroom at night ${ }^{\mathrm{a}}$ & .123 & -.239 & -.127 & -.145 \\
\hline Problems with teeth or gums ${ }^{a}$ & -.080 & $-.223^{\ddagger}$ & -.169 & $-.233^{\ddagger}$ \\
\hline Heartburn $^{\mathrm{a}}$ & .127 & -.189 & .125 & -.117 \\
\hline Problems with constipation $^{\mathrm{a}}$ & -.071 & -.026 & $.227^{\dagger}$ & -.073 \\
\hline $\begin{array}{l}\text { Heart problems now or in the } \\
\text { past }^{\mathrm{a}}\end{array}$ & -.106 & -.051 & $.222^{\ddagger}$ & -.127 \\
\hline Problems breathing while asleep ${ }^{\mathrm{a}}$ & -.031 & $-.264 * \$$ & -.005 & -.078 \\
\hline Shortness of breath ${ }^{\mathrm{a}}$ & .048 & $-.234^{\ddagger}$ & .113 & -.027 \\
\hline Asthma $^{a}$ & $.188^{\dagger}$ & -.081 & .052 & .129 \\
\hline Allergies $^{\mathrm{a}}$ & .060 & -.062 & $.182^{*}$ & $-.206^{\ddagger}$ \\
\hline $\begin{array}{l}\text { Problems with monthly period or } \\
\text { menstrual cycle }\end{array}$ & .064 & $-.320^{+}$ & .229 & .042 \\
\hline Menopause $^{\mathrm{a}}$ & .078 & $-.413 * *$ & .143 & -.036 \\
\hline Other health problems ${ }^{a}$ & .133 & $-.457 * * * *$ & -.167 & .024 \\
\hline Sum of health problems ${ }^{b}$ & .001 & $-.453 * * *+$ & .012 & $-.233^{\ddagger}$ \\
\hline Smokes $^{\mathrm{a}}$ & $-.196^{\ddagger}$ & $198^{\ddagger}$ & .158 & $-.276 *+$ \\
\hline Sum of health risks ${ }^{b}$ & .001 & .035 & .137 & $-.191^{*}$ \\
\hline $\begin{array}{l}\text { Last visit to a health-care } \\
\text { provider }^{c}\end{array}$ & $-.174^{\ddagger}$ & -.038 & -.082 & .047 \\
\hline Last visit to a dentist ${ }^{\mathrm{c}}$ & -.104 & $-.179 \ddagger$ & -.007 & .076 \\
\hline Last visit to an eye specialist $^{\mathrm{c}}$ & $-.267 *$ & -.200 & .010 & -.117 \\
\hline $\begin{array}{l}\text { Last visit to an emergency } \\
\text { department/urgent care center }^{\mathrm{c}}\end{array}$ & -.002 & $-.231^{\ddagger}$ & -.037 & .021 \\
\hline
\end{tabular}


Table 4-13. (continued)

\begin{tabular}{lcccc}
\hline $\begin{array}{l}\text { Self-reported health problem, } \\
\text { health risk, or health-care } \\
\text { utilization }\end{array}$ & $\begin{array}{c}\text { SF-12v2 } \\
\text { PCS } \\
\text { self- } \\
\text { report }\end{array}$ & $\begin{array}{c}\text { SF-12v2 } \\
\text { MCS } \\
\text { self- } \\
\text { report }\end{array}$ & $\begin{array}{c}\text { SF-12v2 } \\
\text { PCS } \\
\text { caregiver- } \\
\text { report }\end{array}$ & $\begin{array}{c}\text { SF-12v2 } \\
\text { MCS } \\
\text { caregiver } \\
\text {-report }\end{array}$ \\
\hline $\begin{array}{l}\text { Last female exam } \\
\text { Has regular primary care }\end{array}$ & -.054 & $-.290^{\ddagger}$ & .225 & -.182 \\
$\begin{array}{l}\text { provider } \\
\text { Resistance to health care }\end{array}$ & -.064 & $.240^{\ddagger}$ & .069 & $.204^{\ddagger}$ \\
\hline
\end{tabular}

Note. Self-reported health problems, health risks, or health-care utilization correlated with a $p$-value $\leq .2$ on one or more of the dependent variables are included in this table ${ }^{\text {a }}$ Point-biseral correlation coefficient ${ }^{\mathrm{b}}$ Pearson Product correlation coefficient 'Spearman's rho correlation coefficient p-values with cut-point of .2 to be included in multiple regression analysis ${ }^{*} \mathrm{p}<.05 ; * * \mathrm{p}<.01 ; * * * \mathrm{p}<.001$ 
Table 4-14. Correlation of the health-related quality of life of adults with Down syndrome via self- and caregiver-report to health problems, health risks, and health-care utilization per caregiver-report

\begin{tabular}{|c|c|c|c|c|}
\hline $\begin{array}{l}\text { Caregiver-reported health } \\
\text { problem, health risk, or } \\
\text { health-care utilization }\end{array}$ & $\begin{array}{c}\text { SF-12v2 } \\
\text { PCS } \\
\text { self- } \\
\text { report }\end{array}$ & $\begin{array}{c}\text { SF-12v2 } \\
\text { MCS } \\
\text { self- } \\
\text { report } \\
\end{array}$ & $\begin{array}{c}\text { SF-12v2 } \\
\text { PCS } \\
\text { caregiver } \\
\text {-report }\end{array}$ & $\begin{array}{c}\text { SF-12v2 } \\
\text { MCS } \\
\text { caregiver- } \\
\text { report } \\
\end{array}$ \\
\hline Trouble walking $^{\mathrm{a}}$ & .073 & -.001 & $-.464 * * *$ & .086 \\
\hline Trouble getting out of chair ${ }^{\mathrm{a}}$ & .119 & .044 & $-.314^{*+!}$ & .022 \\
\hline Aches and pains ${ }^{\mathrm{a}}$ & .050 & -.189 & $-.247^{\ddagger}$ & -.048 \\
\hline Arthritis ${ }^{\mathrm{a}}$ & $-.267 * t$ & -.118 & $-.184^{\ddagger}$ & .093 \\
\hline Dizziness or weak spells ${ }^{a}$ & .078 & -.158 & -.095 & $-.311 * \$$ \\
\hline Headaches $^{\mathrm{a}}$ & .027 & -.159 & $-.214^{\ddagger}$ & $-.174^{\star}$ \\
\hline Problems hearing $^{\mathrm{a}}$ & .001 & $-.188^{\ddagger}$ & -.086 & $-.225^{t}$ \\
\hline Problems seeing $^{\mathrm{a}}$ & -.165 & -.077 & $-.256 *+$ & -.158 \\
\hline $\begin{array}{l}\text { Problems reading or identifying } \\
\text { small objects }^{\mathrm{a}}\end{array}$ & -.075 & $.226^{\ddagger}$ & -.045 & .114 \\
\hline Feel sad or blue ${ }^{a}$ & .112 & $-.354 * * \$$ & -.127 & $-.387 * * *$ \\
\hline Problems remembering $^{\mathrm{a}}$ & .028 & -.026 & -.012 & $-.256^{*}$ \\
\hline Problems with appetite $^{\mathrm{a}}$ & -.162 & -.112 & -.067 & $-.491 * * *+$ \\
\hline Problems with weight ${ }^{\mathrm{a}}$ & -.102 & $-.171^{\ddagger}$ & $-.362 * * *$ & -.022 \\
\hline Thyroid problems $^{\mathrm{a}}$ & .022 & -.035 & $-.229^{\ddagger}$ & .025 \\
\hline Go to the bathroom at night ${ }^{\mathrm{a}}$ & .142 & $-.171^{\dagger}$ & -.062 & -.146 \\
\hline $\begin{array}{l}\text { Number of times going to the } \\
\text { bathroom per night }\end{array}$ & .084 & $-.267 * \hbar$ & -.127 & $-.214^{\ddagger}$ \\
\hline Problems with teeth or gums ${ }^{a}$ & $-.319 *+$ & $-.242^{\ddagger}$ & $-.284 *+$ & -.079 \\
\hline Heartburn ${ }^{\mathrm{a}}$ & .042 & -.064 & $-.176^{\ddagger}$ & -.089 \\
\hline Problems with constipation $^{\mathrm{a}}$ & -.169 & -.014 & -.128 & $-.308 * \hbar$ \\
\hline $\begin{array}{l}\text { Heart problems now or in the } \\
\text { past }^{\mathrm{a}}\end{array}$ & $.204^{\dagger}$ & .061 & .100 & -.104 \\
\hline $\begin{array}{l}\text { Circulatory problems/blood } \\
\text { clots }^{\mathrm{a}}\end{array}$ & $.211^{\ddagger}$ & .042 & $-.358 * *$ & .099 \\
\hline Problems breathing while asleep ${ }^{a}$ & $-.193^{\ddagger}$ & -.156 & -.189 & -.097 \\
\hline Colds often ${ }^{\mathrm{a}}$ & -.254 & .107 & .008 & .014 \\
\hline Problems with coughing $^{\mathrm{a}}$ & -.137 & $.194^{\ddagger}$ & .032 & -.199 \\
\hline Asthma $^{\mathrm{a}}$ & $.221^{\ddagger}$ & $-.228^{+}$ & -.053 & -.011 \\
\hline Allergies $^{\mathrm{a}}$ & $.186^{\dagger}$ & $-.215^{+}$ & $.208^{\ddagger}$ & -.156 \\
\hline $\begin{array}{l}\text { Problems with monthly period or } \\
\text { menstrual cycle }\end{array}$ & .037 & $-.359^{+}$ & .021 & -.017 \\
\hline Menopause $^{\mathrm{a}}$ & .043 & $-.256^{\ddagger}$ & -.095 & .183 \\
\hline Prostate problems ${ }^{\mathrm{a}}$ & -.099 & $-.389 * \$$ & $-.462 * \$$ & $-.439 * \$$ \\
\hline Other health problems $^{\mathrm{a}}$ & .007 & $.181^{*}$ & -.090 & -.019 \\
\hline Sum of health problems ${ }^{b}$ & -.050 & $-.196^{\ddagger}$ & $-.393 * *$ & $-.286 * *$ \\
\hline Drink alcohol $^{\mathrm{a}}$ & $.217^{\ddagger}$ & .158 & $.204^{*}$ & .041 \\
\hline Sum of health risks ${ }^{b}$ & $.217^{\ddagger}$ & .158 & $.204^{*}$ & .041 \\
\hline
\end{tabular}


Table 4-14. (continued)

\begin{tabular}{|c|c|c|c|c|}
\hline $\begin{array}{l}\text { Caregiver-reported health } \\
\text { problem, health risk, or health- } \\
\text { care utilization }\end{array}$ & $\begin{array}{l}\text { SF-12v2 } \\
\text { PCS } \\
\text { self- } \\
\text { report }\end{array}$ & $\begin{array}{l}\text { SF-12v2 } \\
\text { MCS } \\
\text { self- } \\
\text { report }\end{array}$ & $\begin{array}{l}\text { SF-12v2 } \\
\text { PCS } \\
\text { caregiver- } \\
\text { report }\end{array}$ & $\begin{array}{l}\text { SF-12v2 } \\
\text { MCS } \\
\text { caregiver- } \\
\text { report }\end{array}$ \\
\hline $\begin{array}{l}\text { Last visit to a health-care } \\
\text { provider }^{c}\end{array}$ & -.057 & $.198^{\ddagger}$ & -.027 & $-.294 * t$ \\
\hline Last visit to an eye specialist $\mathrm{c}^{\mathrm{c}}$ & $-.242^{\ddagger}$ & .112 & -.018 & $-.267 * t$ \\
\hline $\begin{array}{l}\text { Last visit to an emergency } \\
\text { department or urgent care center }\end{array}$ & .082 & .026 & .112 & $-.297 *$ \\
\hline Last time hearing was checked ${ }^{\mathrm{c}}$ & .104 & .028 & .110 & $-.175^{\ddagger}$ \\
\hline Last $\mathrm{TB}$ test $^{\mathrm{c}}$ & .171 & -.020 & $-.394 * * t$ & $.179^{\ddagger}$ \\
\hline Last flu shot ${ }^{\mathrm{c}}$ & -.153 & $-.214^{\ddagger}$ & -.144 & .001 \\
\hline Last prostate exam ${ }^{\mathrm{c}}$ & .083 & -.186 & -.166 & $-.345^{\dagger}$ \\
\hline $\begin{array}{l}\text { Last mammogram or breast } \\
\text { ultrasound }^{\text {c }}\end{array}$ & $-.272^{+}$ & .172 & -.121 & -.067 \\
\hline Has Medicaid $^{\mathrm{a}}$ & -.089 & .013 & .089 & $-.297 * \hbar$ \\
\hline Has no insurance $^{a}$ & $-.242^{+}$ & .112 & -.018 & $-.267 *$ \\
\hline
\end{tabular}

Note. Caregiver-reported health problems, health risks, or health-care utilization correlated with a p-value $\leq .2$ on one or more of the dependent variables are included in this table

${ }^{a}$ Point-biseral correlation coefficient

${ }^{\mathrm{b}}$ Pearson Product correlation coefficient

${ }^{\mathrm{c}}$ Spearman's rho correlation coefficient

${ }^{\sharp}$-values with cut-point of 2 to be included in multiple regression analysis

$* \mathrm{p}<.05 ; * * \mathrm{p}<.01 ; * * * \mathrm{p}<.001$ 
Table 4-15. Correlation of the health-related quality of life of adults with Down syndrome via self- and caregiver-report to adaptive behavior per caregiver-report

\begin{tabular}{|c|c|c|c|c|}
\hline Vineland-II scale & $\begin{array}{c}\text { SF-12v2 } \\
\text { PCS } \\
\text { self- } \\
\text { report }\end{array}$ & $\begin{array}{c}\text { SF-12v2 } \\
\text { MCS } \\
\text { self- } \\
\text { report }\end{array}$ & $\begin{array}{c}\text { SF-12v2 } \\
\text { PCS } \\
\text { caregiver- } \\
\text { report }\end{array}$ & $\begin{array}{c}\text { SF-12v2 } \\
\text { MCS } \\
\text { caregiver- } \\
\text { report }\end{array}$ \\
\hline $\begin{array}{l}\text { Receptive communication } \\
\text { subdomain v-scale score }\end{array}$ & -.060 & .022 & .093 & $.272 * t$ \\
\hline $\begin{array}{l}\text { Personal daily living skills } \\
\text { subdomain v-scale score }\end{array}$ & -.096 & -.052 & -.034 & $.336^{* * \hbar}$ \\
\hline $\begin{array}{l}\text { Domestic daily living skills } \\
\text { subdomain v-scale score }\end{array}$ & -.053 & .022 & .041 & $.333 * * \hbar$ \\
\hline $\begin{array}{l}\text { Daily living skills domain } \\
\text { standard score }\end{array}$ & -.085 & .010 & .020 & $.276^{* *}$ \\
\hline $\begin{array}{l}\text { Interpersonal relationships } \\
\text { socialization subdomain v-scale } \\
\text { score }\end{array}$ & -.082 & .040 & .031 & $.299 *$ \\
\hline $\begin{array}{l}\text { Coping socialization subdomain v- } \\
\text { scale score }\end{array}$ & -.160 & .115 & .056 & $361 * * t$ \\
\hline $\begin{array}{l}\text { Socialization domain standard } \\
\text { score }\end{array}$ & -.157 & .109 & .034 & $.348 * * \hbar$ \\
\hline $\begin{array}{l}\text { Adaptive behavior composite } \\
\text { standard score }\end{array}$ & -.099 & .095 & .018 & $.285^{*}$ \\
\hline
\end{tabular}

Note. Vineland-II scores correlated with a p-value $\leq .2$ on one or more of the dependent variables are included in this table; Pearson product-moment correlation coefficients p-values with cut-point of .2 to be included in multiple regression analysis $* \mathrm{p}<.05 ; * * \mathrm{p}<.01$ 
the caregiver-reported MCS at or below the cut point to be included in a regression model.

Next, each independent variable correlated to one or more independent variables with a p-value at or below the cut point of . 2 was entered into a stepwise multiple linear regression model with the dependent variable with which it was correlated. Health problems, health risks, and health-care utilization variables that were gender-specific (e.g., menopause, last prostate exam) were omitted because of the decreased number of participants to whom these variables are applicable. Due to the small size of the entire sample in the current study, the regression models for each dependent variable were calculated according to the categorical nature of the independent variable (e.g., demographic characteristic, health problem, health risk, health-care utilization, or Vineland-II score). Additionally, due to high correlation between the three levels of report on the Vineland-II scores, the subdomain scores, domain scores, and adaptive behavior composite score were each analyzed in a separate model to avoid multicollinearity. Significant predictors are reported for self-reported PCS in Table 4-16, self-reported MCS in Table 4-17, caregiver-reported PCS in Table 4-18, and caregiverreported MCS in Table 4-19. Again, because of the sample size, each dependent variable was analyzed in up to six regression models depending on the significance of correlations at or below. 2 in each category of independent variable.

Finally, all independent variables found to predict self-reported PCS scores in the separate categorical models were analyzed together using stepwise multiple linear regression resulting in one final regression model for this dependent variable (Table 4-20). This was repeated for all categorical independent variables found to predict selfreported MCS (Table 4-20), caregiver-reported PCS (Table 4-21), and caregiverreported MCS (Table 4-21).

Table 4-16. Regression models with self-reported physical component summary score as dependent variable

\begin{tabular}{lccc}
\hline Characteristic & Beta & $\boldsymbol{t}$ Statistic & p-Value \\
\hline Demographic characteristic $\left(\mathrm{R}^{2}=.124\right):$ & & & .006 \\
Race of adult with DS & .353 & 2.869 & .006 \\
& & & \\
Health problems per caregiver-report $\left(\mathrm{R}^{2}=.166\right):$ & & .006 \\
Problems with teeth or gums & -.339 & -2.768 & .008 \\
Asthma & .254 & 2.070 & .043 \\
\hline
\end{tabular}

Note. Only significant coefficients reported 
Table 4-17. Regression models with self-reported mental component summary score as dependent variable

\begin{tabular}{lccc}
\hline Characteristic & Beta & t Statistic & p-Value \\
\hline Demographic characteristic $\left(\mathrm{R}^{2}=.074\right):$ & & & .035 \\
$\quad$ Age of caregiver & -.273 & -2.160 & .035 \\
& & & \\
Health problems per self-report $\left(\mathrm{R}^{2}=.333\right):$ & & & .000 \\
$\quad$ Other health problems & -.454 & -4.120 & .000 \\
Feeling sad or blue & -.266 & -2.412 & .019 \\
Problems with teeth or gums & -.249 & -2.256 & .028 \\
& & & \\
Health problems per caregiver-report $\left(\mathrm{R}^{2}=.287\right):$ & & .001 \\
$\quad$ Number of times going to the bathroom at & -.401 & -3.339 & .002 \\
$\quad$ night & & & \\
Allergies & -.332 & -2.827 & .007 \\
Frequent coughing & .317 & 2.665 & .010 \\
Weight problems & -.269 & -2.280 & .027 \\
\hline
\end{tabular}

Note. Only significant coefficients reported

Table 4-18. Regression models with caregiver-reported physical component summary score as dependent variable

\begin{tabular}{lccc}
\hline Characteristic & Beta & t Statistic & p-Value \\
\hline Demographic characteristic $\left(\mathrm{R}^{2}=.210\right):$ & & & .001 \\
$\quad$ State of residence & .372 & 3.154 & .003 \\
Gender of adult with DS & .252 & 2.143 & .036 \\
& & & \\
Health problems per self-report $\left(\mathrm{R}^{2}=.200\right):$ & & & .002 \\
$\quad$ Trouble walking & -.387 & -3.251 & .002 \\
Problems with constipation & .266 & 2.236 & .029 \\
& & & .003 \\
Health-care utilization per self-report $\left(\mathrm{R}^{2}=.144\right):$ & -.380 & -3.127 & .003 \\
$\quad$ & & & .000 \\
Resistance to health care & & & .000 \\
Health problems per caregiver-report $\left(\mathrm{R}^{2}=.315\right):$ & -.431 & -3.916 & .005 \\
$\quad$ Trouble walking & -.319 & -2.892 & \\
Weight problems & &
\end{tabular}

Note. Only significant coefficients reported 
Table 4-19. Regression models with caregiver-reported mental component summary score as dependent variable

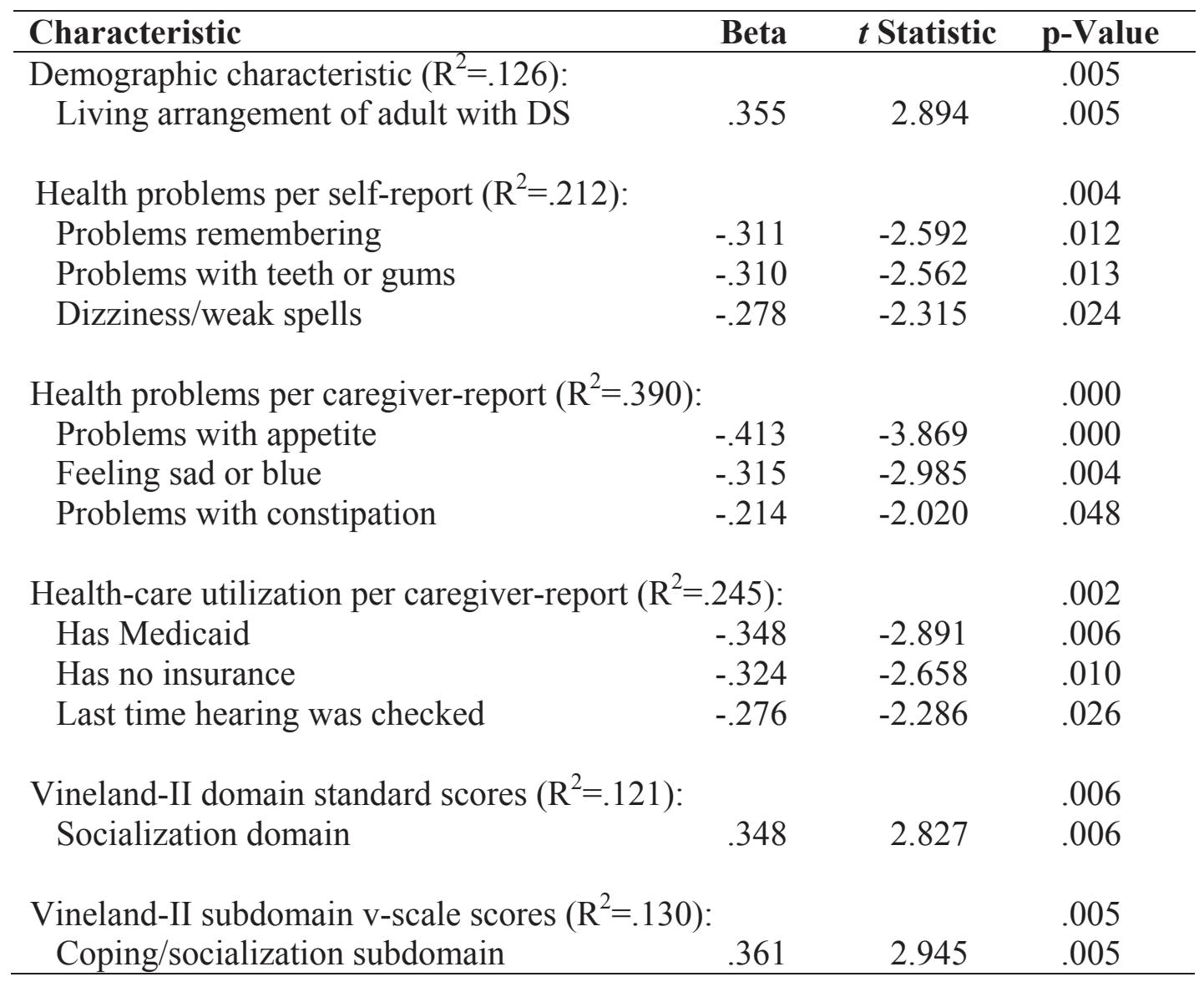

Note. Only significant coefficients reported 
Table 4-20. Regression models for self-reported health-related quality of life

\begin{tabular}{lrrc}
\hline Characteristic & Beta & $\boldsymbol{t}$ Statistic & p-Value \\
\hline Self-reported PCS $\left(\mathrm{R}^{2}=.285\right):$ & & & .000 \\
$\quad$ Race of adult with DS & .347 & 3.038 & .004 \\
$\quad$ Problems with teeth or gums per caregiver & -.344 & -3.006 & .004 \\
$\quad$ Asthma per caregiver & .238 & 2.081 & .042 \\
& & & \\
Self-reported MCS ( $\left.{ }^{2}=.400\right):$ & -.392 & -3.643 & .000 \\
$\quad$ Other health problems per self & -.331 & -3.044 & .004 \\
$\quad$ Feeling sad or blue per self & -.310 & -2.838 & .006 \\
$\quad$ Number of times going to the bathroom at & & & \\
$\quad$ night per caregiver & -.221 & -2.054 & .045 \\
$\quad$ Allergies per caregiver & &
\end{tabular}

Note. Only significant coefficients reported

Table 4-21. Regression models for caregiver-reported health-related quality of life

\begin{tabular}{lrrc}
\hline Characteristic & Beta & $\boldsymbol{t}$ Statistic & p-Value \\
\hline Caregiver-reported PCS $\left(\mathrm{R}^{2}=.540\right):$ & & & .000 \\
Trouble walking per caregiver & -.326 & -3.366 & .001 \\
Resistance to health care per self & -.285 & -3.028 & .004 \\
State of residence & .267 & 2.817 & .007 \\
Weight problems per caregiver & -.252 & -2.690 & .009 \\
Trouble walking per self & -.242 & -2.519 & .015 \\
& & & \\
Caregiver-reported MCS $\left(\mathrm{R}^{2}=.391\right):$ & & & .000 \\
Problems with appetite per caregiver & -.516 & -4.977 & .000 \\
Living arrangement of adult with DS & .388 & 3.747 & .000 \\
\hline
\end{tabular}

Note. Only significant coefficients reported 


\section{CHAPTER 5. DISCUSSION}

This study examined the health-related quality of life (HRQOL) and factors associated with the HRQOL of adults with Down syndrome (DS) as reported by adults with DS and their caregivers. This chapter presents a discussion of the results and the implications of the study.

\section{Demographics}

The respondents in this study were 60 adults with DS and 60 caregivers living in Tennessee, Alabama, Mississippi, and Florida. The adults with DS who participated were 30 women and 30 men aged 18 years and older with a mean age of 30.83 years. The gender division is similar to that in existing HRQOL studies with adults and children with DS (Bertoli et al., 2011; van Gameren-Oosterom et al., 2011; Verstegen et al., 2013). The participants in the current study were largely Caucasian (91.7\%) with low representation of African-American participants (6.7\%) and American/Hispanic participants (1.7\%). This ethnic distribution is not consistent with findings from the 1997-2005 National Health Interview Survey which included a sample of individuals with DS that was Caucasian (79\%), African-American (15.6\%), and other ethnicities (7.2\%; Schieve, Boulet, Boyle, Rasmussen, \& Schendel, 2009). This discrepancy may be attributed to the network or word-of-mouth recruitment technique used in the current study.

Most adults with DS in this study lived at home with family which exceeds the reported number found in a national study of siblings of adults with DS and autism (Hodapp \& Urbano, 2007). Perhaps this difference is also attributable to recruitment methods, a higher socio-economic status of the sample, or possibly the participant criterion in the current study of the adult with DS residing in the community. One adult with DS in the current study owned a home that was shared with two other individuals with intellectual and/or developmental disability (IDD). This man employed supported living staff around the clock. Two other adults with DS had apartments within or adjacent to the families' homes, one with an outside entrance into her apartment, in addition to an entrance from within the home.

Most adults with DS in the current study had graduated from high school, and more than half held employment or volunteered regularly, which is slightly less than the 59.9\% identified by Hodapp and Urbano (2007). This difference could be explained by recruitment technique, a higher socio-economic status of the sample, or possibly the geographical area represented in the current study. However, only $8.3 \%$ of the participants with DS in the current study worked in a sheltered/supported workshop environment compared to $21.5 \%$ of the participants with DS in the sibling study (Hodapp $\&$ Urbano, 2007). This difference can perhaps be explained by an increased availability of supported or independent employment for individuals with DS in the time between the studies or in the region studied (Taylor \& Seltzer, 2012) or, again, by the recruitment 
method. In 2009, 1\% of recipients of day and employment services in Alabama participated in facility-based work such as that occurring in sheltered workshops where the majority of employees/participants have a disability (Butterworth et al., 2012). In the same report, $5 \%$ of recipients of day and employment services participated in integrated employment which typically occurs in a community setting and includes supported and independent (competitive) employment. These data were not available for the other states in the current study. To compare this with the data from the current study, of the 23 adults with DS who were residents of Alabama, 30\% were employed, $4.3 \%$ in facilitybased settings and $8.6 \%$ in integrated settings, and $26.1 \%$ volunteered. While this does not exactly reflect the same results as the report by Butterworth et al., it does show that more individuals in the current study have integrated employment than facility-based employment, which is consistent with the findings of Butterworth et al. The adults with DS in this study worked in a variety of jobs, including working in cafeterias/restaurants $(6.7 \%)$, retail $(5 \%)$, grocery stores $(3.3 \%)$, and offices as office assistants $(3.3 \%)$. Adults with DS participated in volunteer activities at church and a food bank, a non-profit foundation, a golf course, horse stables, and a local high school football program. Other daily activities of adults with DS in the current study included dance class $(6.7 \%)$, routine chores and babysitting (3.3\%), and language classes (3.3\%). Additionally, at least one adult with DS had several routine weekly activities that occurred on the same day each week, such as lessons and social gatherings. Almost all adults with DS had multiple routine weekly activities, increasing the likelihood that these participants are actively involved in their communities.

The caregivers in the current study were primarily female, which is consistent with other research involving caregiver-respondents of adults with DS (Esbensen, Seltzer, \& Krauss, 2008; Kaye, Fiske, Bower, Newton, \& Fenlon, 2005; Mahy, Shields, Taylor, \& Dodd, 2010). The caregivers' mean age was 60.6 years, with a range of $44-79$ years. All but one caregiver in this study were related to the adults with DS, and all but a few of these were the parent of the adult with DS in that dyad. The closeness of these relationships contributes strength to this study based on the extensive history between the dyad and the caregivers' knowledge regarding the thoughts and feelings of the adults with DS regarding their health. These characteristics are consistent with findings in the study of caregivers of adults with DS by Kaye et al. In the current study, the caregivers were primarily married and employed. More than half of the caregivers had a college degree or higher, and none had less than a high-school education. The caregivers in the current study had higher levels of education than in a study comparing adults with DS and adults with autism (Esbensen, Bishop, Seltzer, Greenberg, \& Taylor, 2010), which could impair the generalizability of the results. Out of the mothers of 70 adults with DS in the referent study, 22.9\% had less than a high-school education, $37.1 \%$ had a highschool education, $21.4 \%$ had some college education, and $18.6 \%$ had graduated from college and possibly continued their education. 


\section{Specific Aim One}

The first specific aim was to measure the HRQOL of adults with DS. The following research question relates to the first specific aim:

Research Question 1 What is the HRQOL of adults with DS as reported by:

a) adults with DS?

b) caregivers of adults with DS?

The mean scores from the QualityMetric Short Form-12v2 Health Survey (SF12v2) of self-reported and caregiver-reported HRQOL of adults with DS exceeded the U.S. mean of 50 (Saris-Baglama et al., 2011). This included the norm-based scores as well as the physical component summary (PCS) and mental component summary (MCS) scores. This finding confirms the first hypothesis made at the outset of this study that the means from self-reported PCS and MCS would be above average, and means of the caregiver-reported PCS and MCS would be average to above average.

In the extant literature related to HRQOL of individuals with DS, Bertoli et al. (2011) determined that the variables similar to HRQOL concepts were very low for adults with DS. In research on the HRQOL of children with DS, van Gameren-Oosterom et al. (2011) queried caregivers and reported that children with DS scored lower than the normbased scores of children without DS in multiple scales. The findings in the current study do not corroborate either of these previous studies because the adults with DS in the current study had greater-than-average self-reported and caregiver-reported HRQOL scores on all scales of the SF-12v2, including the PCS and MCS. Perhaps the HRQOL of adults with DS was superior in this study because the sample had more opportunities to attend school and to work or volunteer in the community, two deficits cited by Bertoli et al. to rationalize the very low HRQOL assessment reported.

Because the adults with DS participating in this study generally had satisfactory health and health care and participated in the communities in which they lived, it is logical that they and their caregivers scored physical and mental HRQOL above average. The adults with DS had generally pleasant, positive attitudes, and were enthusiastic about their lives and activities, such as their work or volunteerism, school, or dance. Very few seemed preoccupied with or excessively concerned about health problems or access to care. Perhaps the findings would be different if the sample included or focused on adults with DS who lived in skilled-care facilities. Additionally, during the data collection sessions, the semi-structured interviews about the health problems, health risks, and health-care utilization of the adults with DS took place prior to the verbal completion of the SF-12v2. The order of administration of these two methods of data collection could perhaps affect the results of the SF-12v2 since adults with DS had been reflecting on their health immediately before answering questions about the impact of health on their lives. 


\section{Specific Aim Two}

The second specific aim was to compare the HRQOL data obtained via self-report to that obtained via caregiver-report. The following research question relates to the second specific aim:

Research Question 2 What are the associations between the HRQOL of adults with DS as reported by adults with DS and their caregivers?

There were no associations identified between self-reported and caregiverreported PCS or MCS of adults with DS. The second hypothesis in this study was that a difference would be detected between the mean of self- and caregiver-reported PCS and MCS. Because no statistical difference was identified between self-reported and caregiver-reported PCS ( $p=.590)$ and MCS ( $p=.663)$ scores, the second hypothesis is not supported by this study. The only significant difference was between self- and caregiverreported SF-12v2 role physical norm-based scores $(\mathrm{p}=.018)$, which could be because this score is related to how physical health affects other facets of an individual's usual role (Saris-Baglama et al., 2011; Ware et al., 2007). The significant correlation between selfreported and caregiver-reported physical functioning norm-based scores $(p=.003)$ was not supported by prior research. However, physical functioning is an observable dimension and can be accurately reported by a caregiver (Brown, 1998; Guyatt, Feeny, \& Patrick, 1993).

The third hypothesis that the SF-12v2 could accurately measure the HRQOL of adults with DS can be supported by the convergent validity and discriminant validity estimates obtained from adults with DS, which were acceptable. Therefore this instrument was applicable to this study.

The adults with DS in this study had close, solid relationships with their caregivers, and many spent a considerable amount of time with their caregivers on a daily basis at home and during activities. This supports the principle of linked-lives of the Life Course Perspective (LCP) theoretical framework. The degree of closeness and time spent together identified in this study can contribute to insight and understanding of each other's thoughts and feelings, which could explain why there was no significant difference in the PCS or MCS scores indicating HRQOL of adults with DS provided by self-report and caregiver-report.

Finally, Thorne and Paterson (1998) developed a concept they termed health within illness. This occurs when an individual with chronic health problems has a positive outlook regarding his/her health, despite the health problem or disability. Although a disability is not an illness, this concept is applicable because of the challenges which individuals with disabilities encounter. Perhaps the adults with DS and their caregivers who participated in this study are demonstrating mastery of this concept. 


\section{Specific Aim Three}

The third specific aim was to learn more about the health of adults with DS obtained via self-report compared to that via caregiver-report. The following research question relates to the third specific aim:

Research Question 3 What are the associations between health problems, health risks, and health-care utilization of adults with DS as reported by adults with DS and their caregivers?

\section{Health problems of adults with Down syndrome}

Alzheimer's disease/dementia. Diagnosis of Alzheimer's disease (AD) was not a specific question posed to adults with DS during the interviews, nor was it asked directly on the Health Questionnaire since the construct of the questionnaire focused more on symptoms experienced by the adults with DS and less on diagnoses (Wilson \& Cleary, 1995). However, three adults with DS and 18 caregivers indicated that the adult with DS had problems remembering. Additionally, one adult with DS had severe dementia, and one was diagnosed with AD according to the respective caregivers. The individual with dementia was the oldest participant with DS (61 years old), and the individual with $\mathrm{AD}$ was the second oldest (51 years old). One 50-year-old did not report problems with memory, but this adult's caregiver reported the adult with DS had problems with memory. According to Bosch (2003) signs of AD begin to appear in close to $10 \%$ of adults with DS in their forties. Memory was the only variable in the current study that can be considered symptomatic of AD or dementia, often because adults with DS tend to exhibit atypical symptoms such as behavior and mood disturbances (Bosch, 2003). Of the eight adults with DS between the ages of 40-49 years, one had selfreported problems with memory, and four had caregiver-reported problems with memory. These findings support Bosch's assertion about early signs of AD, and both self- and caregiver-report exceed the estimated percentage.

Although an association between self-reported and caregiver-reported problems with memory was not found, there was a significant difference of the means between the two. This is likely due to the number of adults with DS who reported problems $(n=3)$ compared to the number of caregivers who reported problems $(n=18)$. This may result from the lack of specificity in the interview-method of data collection from the adults with DS.

Cancer. Interestingly, all adults with DS and all caregivers denied issues with cancer for the adults with DS. This may be a chance finding, or it might indicate the question was not worded clearly to ascertain the occurrence of cancer at any point during the lifetime of the individual with DS since the types of leukemia associated with DS often occur during early childhood (Bosch, 2003; Esbensen, Seltzer, \& Greenberg, 2007; 
Kerins, Petrovic, Bruder, \& Gruman, 2008; Shimizu, Engel, \& Yamamoto, 2008; Wiseman, Alford, Tybulewicz, \& Fisher, 2009; Xavier, Ge, \& Taub, 2010). The findings in this study are consistent with an earlier report of the decreased incidence of cancer noted by Crocker (2006a).

Cardiovascular disease. Seven adults with DS and 32 caregivers reported cardiac problems at some point during the lifetime of the individual with DS. Because all cardiac problems reported by caregivers were congenital, this finding confirms the 4050\% risk in extant literature (Bosch, 2003; Cohen, 1999; Esbensen et al., 2007; Nehring $\&$ Betz, 2010). Four adults with DS in the current study stated that they had some type of heart surgery. One adult with DS specifically stated that the surgery was for a hole in her heart around 18 years of age. Another reported having multiple surgeries including a cardiac pacemaker placement. An adult with DS stated that the open-heart surgery included a heart catheterization. Three adults with DS, including the adult who had surgery around 18 years of age indicated that the heart problems occurred during childhood and gave them no problems as adults. One adult with DS reported having problems with tachycardia and shortness of breath when "getting hot". The more common cardiac problems reported by the caregivers were ventricular septal defects $(10 \%)$, heart murmurs $(8.3 \%)$, and atrioventricular canal defects $(5 \%)$. Surgical intervention was required for at least 17 adults with DS according to the caregivers, and two adults with DS were pacemaker-dependent at the time of data collection.

There was no association between self- and caregiver-reported heart problems, but there was a strongly significant difference between the means of the two. Again, this is likely because of low self-report $(n=7)$ due to lack of specificity and much higher caregiver-report of cardiac problems $(n=32)$. Additionally, the caregivers provided more specific information about the types of cardiac problems.

Although two adults with DS and two caregivers reported adults with DS having problems with high blood pressure, only one dyad agreed in their reports, which resulted in a positive correlation. The low report of high blood pressure supports prior research that hypertension does not occur frequently in individuals with DS (Kerins et al., 2008).

The vascular problems reported by adults with DS included an occurrence of blood clots $(1.7 \%)$ and of varicose veins $(1.7 \%)$. These reports were consistent with reports given by their caregivers. One other caregiver reported an occurrence of varicose veins totaling two adults with this problem (3.3\%). Additionally, one caregiver reported an occurrence of bilateral pulmonary emboli, and one reported persistent fetal circulation at birth which was corrected with endotracheal intubation. There were no significant associations found among the self-reported and caregiver-reported vascular problems. No extant literature identifies the risk of vascular problems or varicose veins in adults with DS. 
Depression. Feeling sad or blue is a symptom of depression in individuals with and without DS (McGuire \& Chicoine, 2006). Seventeen adults with DS (28\%) and six caregivers $(10 \%)$ reported adults with DS feeling sad or blue, resulting in a significant difference between the means of self- and caregiver-report. Approximately 20\% of individuals in the United States experience depression some time during their lives, and McGuire and Chicoine (2006) stated that the expected rate of depression in adults with DS surpasses this. Kerins et al. (2008) found that 18\% of adults with DS reported depression. In the current study, the caregiver-reported depressive symptoms of adults with DS feeling sad or blue were lower than the rate reported by Kerins et al. as well as McGuire and Chicoine's anticipated rate of adults with DS and without DS. However, self-reported depressive symptoms were consistent with McGuire and Chicoine's estimates and surpassed the findings by Kerins et al. When asked about feelings of sadness, most adults who answered with a positive response spoke of a particular event that caused sadness or crying when they thought about it, most commonly the death of a loved one. It was unclear to the investigator whether these depressive symptoms occur frequently enough to warrant a diagnosis of depression, or if the adults with DS were identifying with a situation that caused them to feel sad or blue. The associations between self- or caregiver-reported PCS of adults with DS and depression were not significant. Perhaps depression occurring in adults with DS is isolated to mental or emotional concerns with no physical sequelae. These findings support further investigation into depression in adults with DS.

Endocrine disorders. Seventeen adults with DS and 32 caregivers reported adults with DS who have thyroid problems. There were both a significant difference and a significant correlation between self- and caregiver-report. According to Esbensen (2010), thyroid abnormalities occur in $35-40 \%$ of adults with DS. In the current study, fewer adults with DS self-reported thyroid disorders than this estimate, but the caregiverreport was higher. Once again, the lack of specificity in the interviews of the adults with DS potentially contributed to this lower percentage of self-report.

While no adults with DS reported having diabetes, one participant stated she takes metformin (an oral medication generally prescribed for Type 2 diabetes) to help her blood sugar, but she denied having diabetes when asked specfically. The caregiver of that adult with DS also denied diabetes as a problem for this participant. Three caregivers stated that adults with DS had diabetes. No significant associations were found between self- and caregiver-reported diabetes. The Health Questionnaire did not specify the difference between Type 1 or Type 2 diabetes. The percentage of caregiverreported diabetes in this study is consistent with the Type 1 diabetes range of $1.4-10.6 \%$ that was reported in individuals with DS living in Scotland (Anwar, Walker, \& Frier, 1998).

Gastrointestinal disorders. Heartburn of adults with DS, which is a symptom of gastroesophageal reflux disease (Estores, 2014), was reported by six adults with DS and 10 caregivers which supports prior research (Kerins et al., 2008). There was no 
significant difference between the means of self- and caregiver-report, but a positive correlation was found.

Problems with weight and appetite of adults with DS were reported by 19 and 15 adults with DS respectively, and 26 and four caregivers respectively. Self- and caregiverreported weight problems had a significant positive correlation, but no significant difference between the means. Self- and caregiver-reported appetite problems had a significant difference between the means, but not a significant correlation. Weight problems in individuals with DS are close to three times more common than in individuals without DS (Esbensen, 2010). Esbensen reported that women with DS have a $56-96 \%$ chance of being overweight, and men have a $45-79 \%$ chance. In the current study, weight problems occurred in $40 \%$ of women with DS via self-report and $63.3 \%$ via caregiver-report. The rate of self-reported problems with weight is lower than the rate of overweight women with DS, but the rate of caregiver-reported problems falls within the estimated occurrence. Men in the current study had problems with weight based on selfreport $(23.3 \%)$ and caregiver-report $(23.3 \%)$. This finding is lower than the estimated rates of overweight men with DS, which could be related to more awareness of potential weight problems among the men with DS and their caregivers, and/or a more physically active group of men in the current study.

Six adults with DS and 14 caregivers reported that adults with DS had problems with constipation. There were no significant associations between reports. Although extant literature reports increased occurrence of constipation, no incidence rates were provided (Bosch, 2003; Crocker, 2006a). Celiac disease was not specifically discussed during the health interview or mentioned on the Health Questionnaire because it is a diagnosis; however, one caregiver reported that the adult with DS had a gluten allergy. This potential $1.7 \%$ occurrence of celiac disease is less than the estimated $4-17 \%$ occurrence in individuals with DS (Bosch, 2003).

Thirteen adults with DS and 18 caregivers reported dental problems in the adults with DS. No significant differences were found between the means of self- and caregiver-report, but a significant positive correlation was found. Cohen (1999) reported that dental problems are increased in individuals with DS due to small mouths and propensity for mouth breathing secondary to small nasal passages.

Immune deficiency. No specific immune deficiency was identified in the study, and only one adult with DS and five caregivers reported adults with DS having frequent colds, which is inconsistent with prior research (Bosch, 2003; Cohen, 1999; Esbensen et al., 2007; Kerins et al., 2008; McCarron, Gill, McCallion, \& Begley, 2005; Service \& Hahn, 2003; Virji-Babul, Eichmann, Kisly, Down, \& Haslam, 2007). No significant associations were found. However, 22 adults with DS and 29 caregivers reported allergies in adults with DS with a significant difference between the means of self- and caregiver-report. 
One adult with DS and her caregiver reported lupus-like symptoms. This caregiver reported that, although it was undiagnosed as lupus at the time of data collection, a medication for lupus was providing some relief from the symptoms. There are no incidences of immune deficiencies, allergies, or auto-immune disorders such as lupus among individuals with DS reported in the extant literature.

Mental health disorders. Symptoms for other mental health problems besides depression were not specific questions in the interview or on the Health Questionnaire, but were included by the participants. Six adults with DS reported anger problems or temper issues when asked about health problems, indicating that adults with DS may include mental health as part of their overall health status. Although there seems to be a stereotype of individuals with DS being loving, kind, and cooperative, they report experiencing stress, anxiety, and anger, which they perceived as a health problem. Three adults with DS and one caregiver reported problems with anxiety or stress, one adult with DS and one caregiver reported psychosis, and one caregiver reported repetitive behaviors. Kerins et al. (2008) found that $7 \%$ of adults with DS have anxiety, and $17 \%$ have one or more other mental illnesses. The current study found less anxiety and other mental illness, although comparison is challenging since the other types of illnesses were not specific in the referent study, and because the data from the current study were not generated from specific questions about mental health.

Musculoskeletal disorders. Osteoporosis was not asked about or reported by adults with DS or caregivers. However two adults with DS mentioned having low Vitamin D levels, which can contribute to osteoporosis (Crowther-Radulewicz \& McCance, 2010). Kerins et al. (2008) noted that 24\% of adults with DS had osteoporosis. This indicates a need to investigate osteoporosis in adults with DS in future research.

Adults with DS and caregivers reported osteoarthritis (OA), aches and pains, and trouble walking in adults with DS. Additionally, two caregivers reported adults with DS had trouble getting up out of a chair. It is possible that OA and aches and pains are interchangeable in the conversations of adults with DS since neither was specifically asked about in the interviews, therefore the multiple regression models were calculated with these separately when necessary to avoid multicollinearity. One caregiver reported an adult with DS having flat feet, which is consistent with prior research (Thomas et al., 2011). Kerins et al. (2008) found that $13 \%$ of adults with DS had OA and/or other musculoskeletal problems. Combining OA, aches and pains, and trouble walking, the findings from the current study were notably higher for musculoskeletal problems. Unfortunately, aside from OA, the types and extent of musculoskeletal problems were not specified by Kerins et al., which prevents direct comparison.

Neurological disorders. Only one caregiver reported a history of seizures in an adult with DS. This was reported for the 51-year-old adult with DS who had been 
diagnosed with $\mathrm{AD}$, potentially supporting an association between seizures and $\mathrm{AD}$ (McCarron et al., 2005).

Headaches were reported by 12 adults with DS and 12 caregivers with a positive correlation. Dizziness or weak spells were reported by four adults with DS and seven caregivers. No significant associations were identified. While headaches can occur for many reasons, they could possibly be associated with obstructive sleep apnea (OSA; Bosch, 2003). Headaches can also be associated with hypertension, cardiac disease, and hypothyroidism (Service \& Hahn, 2003). There is no extant literature to indicate other causes of increased incidence of headaches in adults with DS.

Renal disease. No renal disease was reported by adults with DS or caregivers. One adult with DS reported bladder problems, and one adult with DS reported needing to get up to use the bathroom during the night. Eleven caregivers reported adults with DS using the bathroom at night, with a significant difference of means of self- and caregiverreport. For those adults who did get up during the night to use the bathroom, the mean number of times per night was 1.8 per caregiver-report. No information on increased occurrences of nocturia or bladder problems was available in the extant literature.

Reproductive disorder. No specific reproductive health problems were identified by adults with DS or their caregivers, and no adults reported being the parent of a child. Of the 30 women with DS in the study, 13 reported having irregular, heavy or painful menstrual cycles, with 14 caregivers reporting the same. A positive correlation was found between reports. A positive correlation was also found between the two women with DS and five caregivers who reported women with DS experiencing menopause, including one woman who had a hysterectomy at age 24 . The mean age of menopause for the remaining four participants was 37.75 years old. According to Alford and Nurudeen (2013) the mean age all women experience menopause is 51 years old. The findings in this study, therefore, support earlier findings that menopause occurs at a younger age in women with DS (Bosch, 2003; Service \& Hahn, 2003).

Respiratory disease. There was a low incidence of asthma in the adults with DS in this study with one self-report and two caregiver-reports. There was a positive correlation among these reports. Seven adults with DS and 11 caregivers reported problems with coughing among adults with DS with no significant associations. Finally, one adult with DS and three caregivers reported shortness of breath among adults with DS with no significant associations. Kerins et al. (2008) found that $18 \%$ of adults with DS had pulmonary condition(s), which included asthma and pneumonia. However, because the conditions are not differentiated in their study, comparison to the current sample is not possible. Roizen et al. (2013) found that $31.8 \%$ of children with DS had asthma/reactive airway disease. Again, the samples are not comparable because, in this instance, the participants with DS were children. Five percent of adults with DS in the 
current study reported having a history of pneumonia. This finding warrants further investigation as a health problem among adults with DS.

Sensory impairments. Ear problems, including frequent cerumen accumulation, infections, and a history of surgery, were reported by seven adults with DS and four caregivers. This was not a specific question in the health interview or on the Health Questionnaire, but rather was information contributed by the participants. Additionally, two adults with DS and one caregiver reported a history of tympanostomy tubes for the adult with DS. Hearing problems were reported by three adults with DS and 18 caregivers. The difference between the means of the two report methods was significant. Families of 211 school-aged children with DS living at home in Western Australia reported that $47.6 \%$ of the children with DS had been diagnosed with otitis media and $10.5 \%$ with hearing loss (Leonard, Bower, Petterson, \& Leonard, 1999). There were appreciably more ear problems in the study of children, but more ear problems occur during childhood when the anatomy of the ear is smaller (Crocker, 2006a; Virji-Babul et al., 2007). Esbensen (2010) reported that between 12-72\% of adults with DS have hearing loss. Adults with DS in this study self-reported a lower incidence of hearing loss than reported by Esbensen, while caregivers reported a rate within the range. The higher specificity of the Health Questionnaire completed by caregivers compared to the interview questions for adults with DS could contribute to this difference.

Ocular problems reported were difficulty seeing, reported by 22 adults with DS and 24 caregivers with no significant association; and presbyopia, or trouble reading or seeing small objects, reported by three adults with DS and 13 caregivers. There was a significant difference of the means of self-reported and caregiver-reported presbyopia. Esbensen (2010) reported that difficulty with vision occurs in $18 \%$ of adults with DS who are $30-39$ years old, $28 \%$ of adults with DS who are $40-49$ years old, and $45 \%$ of adults with DS who are 50-59 years old. Of the 13 adults with DS between the ages of 30-39 in the current study, six self-reported some vision problem (difficulty seeing and/or presbyopia), and two caregivers reported some vision problem for adults with DS. In this instance, the adults with DS report a higher incidence than the literature, and the caregivers report is lower. Of the eight adults with DS who were 40-49, three reported some vision problem which was the same percentage as caregiver-reported vision problems. This represents a higher incidence than the extant literature. Finally, in the three adults with DS who were 50-59, none reported vision problems, but three caregivers reported vision problems for the adults with DS. Again, this is higher than reported in previous research.

Skin conditions. Although not a specific question in the health interview or on the Health Questionnaire, skin problems were reported by six adults with DS and seven caregivers. These conditions included three self-reported and three caregiver-reported issues with extreme dryness, which is consistent with the literature (Bosch, 2003; Kerins et al., 2008; Service \& Hahn, 2003). Other conditions included psoriasis reported by two 
adults with DS and two caregivers, warts reported by one adult with DS, eczema reported by one caregiver, and nail fungus reported by one caregiver.

Sleep disorders. Difficulty breathing while asleep can be a symptom of OSA (Huether, 2010). This symptom was reported by four adults with DS and 15 caregivers with a significant positive correlation. Both reports were substantially lower than prior research which indicated that up to $94 \%$ of adults with DS have OSA (Esbensen, 2010). Additionally, self-reported difficulty breathing while asleep and self-reported problems with weight were not significantly correlated, nor were caregiver-reported difficulty breathing while asleep correlated with caregiver-reported weight problems. This does not support the notion that being overweight is associated with OSA in adults with DS (Cohen, 1999). No other sleep problems were reported.

Summary. Health problems that were more frequently reported by adults with DS were symptoms that are generally experienced or felt, such as pain and depression, that may not be visible or obvious to others. Additionally, adults with DS reported emotional problems, such as anger and anxiety when asked about other health problems. The caregivers more frequently reported health problems with symptoms that are observable, such as weight problems, or those which require more medical intervention, such as thyroid problems or cardiac problems.

\section{Health risks of adults with Down syndrome}

The primary health risk for the adults with DS in the current study was consumption of alcohol. As discussed previously, most adults with DS in this study stated they consume alcohol on an infrequent basis. However, the four who indicated that they had one or more drinks per day could be at risk for alcohol abuse and addiction, which has been reported in individuals with DS (McGillicuddy, 2006; McGuire \& Chicoine, 2006). No questions were included in the interviews or on the Health Questionnaire to assess for alcohol addiction. The mean caregiver-reported alcohol consumption differed significantly from self-reported consumption, and a positive correlation was identified. The mean frequency of consumption reported by caregivers was also different, with only one caregiver reporting daily consumption of alcohol by the adult with DS. This could indicate that the other three adults with DS who stated they consumed alcohol daily could be doing so without the knowledge of the caregiver.

As stated previously, the two adults with DS who stated that they smoke tobacco indicated that they had smoked on rare occasions, and did not do so regularly. Both denied smoking daily, and both answered that they had never purchased or been given an entire pack of cigarettes. One woman stated that she occasionally smoked when she went out with friends, which was not very often. However, she resolutely stated that, as an adult, it was her decision to smoke if she wished. None of the caregivers reported adults 
with DS smoking. No adult with DS or caregiver reported exposure to second-hand smoke.

The only other health risk that was mentioned was the use of oral contraceptives to regulate and lessen complications with menses. No adults with DS mentioned that they were sexually active, and no caregivers answered "yes" to that question on the Health Questionnaire. However, one caregiver wrote that is was believed the adult with DS had been sexually active during a previous relationship. Regarding birth control, however, the adults with DS and their caregivers were specific that birth control was not used as a form of contraception. There was a positive correlation identified between selfand caregiver-report of contraceptive use.

Comprehension of meaning might have influenced the responses of the adults with DS when asked questions about drinking alcohol and smoking tobacco. The investigator's perception was that positive reports of alcohol consumption and cigarette smoking did not usually indicate regular or habitual drinking or smoking. Rather, the use of one or both of these substances was generally infrequent or had been experimented with on rare occasions. This would explain the discrepancy between self- and caregiverreport. However, infrequent use of either alcohol or tobacco, both known to be highly addictive, can lead to habitual use and have a negative impact on an individual's health.

\section{Health-care utilization by adults with Down syndrome}

During the interviews, more than half of the adults with DS and almost all caregivers stated that the adults with DS have a primary-care provider, which resulted in a significant difference among the self- and caregiver-report. Most adults with DS were unable to recall the last time they visited a particular health-care provider or had a specific exam. The majority of all answers were mentioned without timeframe or not mentioned, although the investigator prompted the adults with DS to try to specify time frames during the interviews. This is consistent with findings of long-term explicit memory impairment in individuals with DS (Lott, 2012; Vicari, Bellucci, \& Carlesimo, 2000). The caregiver responses provided more detail for these questions. However, this may be partially attributed to the request for specificity within the Health Questionnaire. Although the format of responses had to be changed to "yes" or "no" to facilitate statistical analysis, all but two questions had a significant difference using the McNemar tests to compare self-reported and caregiver-reported health-care utilization by adults with DS. Since the Health Interview Guide included questions to specifically ask adults with DS about health-care providers, it can be rationalized that, regardless of the problems involved in quantitizing qualitative data to facilitate these comparisons, the caregivers do generally provide more specificity in the area of health-care utilization by adults with DS.

The majority of adults with DS acknowledged participation in basic recommended health assessments and maintenance (e.g., primary medical, dental, ophthalmologic, and audiologic), although they did not indicate the frequency. 
According to the caregivers, the majority of adults with DS had visited a health-care provider, dentist, and eye specialist within the prior year and had their hearing evaluated within the prior two years. The McNemar test of the last visit to a health-care provider via self-report and caregiver-report was one of the two items measuring health-care utilization that was not significant. The only correlation for self-reported and caregiverreported health-care utilization was the last visit to an eye specialist by the adult with DS. However, there was still a significant McNemar test for the difference between the two reports. An annual routine physical is recommended for adults with DS (Chicoine, McGuire, Bilodeau, \& Dominiak, 2010). Cohen (1999) and Chicoine et al. recommend dental exams every six months and ophthalmologic and auditory evaluations every one to two years for adults with DS.

Most adults with DS did not mention diagnostic or evaluative assessments that are strongly recommended for individuals with DS such as an annual thyroid assessment and a cervical spine x-ray every 10 years (Chicoine et al., 2010). However, caregivers indicated that most adults with DS had a thyroid assessment within the past year and a cervical spine x-ray during their lifetimes but more than two years prior.

Routine cancer screenings were not addressed by most adults with DS, including a gynecological exam and breast exam for women or a prostate or testicular exam for men. Caregivers reported that slightly more than half of the women with DS had a Papanicolaou exam (Pap smear) or internal exam at some point; therefore almost half of the women had never had a Pap smear. Cohen (1999) recommended a Pap smear every one to three years for sexually active women or a single-finger bimanual vaginal exam with cytology for women not sexually active. If a woman is not sexually active, has no symptoms of cervical cancer or lesions, and has had two normal annual Pap smears, Chicoine et al. (2010) recommend a Pap smear every three years. A pelvic ultrasound is an acceptable alternative for women who refuse or cannot tolerate an internal exam (Cohen, 1999). Caregivers reported that a large majority of women with DS in this study had never had a breast exam or mammogram. Chicoine et al. recommend a physical breast exam every year and mammography every year after the age of 40 . Caregivers reported that most men with DS had a testicular exam within the prior two years. For men with DS, Chicoine et al. recommend a testicular exam once a year. Finally, caregivers reported that most men with DS had never had a prostate exam. There are no specific recommendations for screening for prostate cancer for men with DS, and the U.S. Preventive Services Task Force (USPSTF; 2012a) does not recommend prostate cancer screening currently for men. While these recommendations specifically involve the serum prostate-specific antigen test for screening, supporting documents include not recommending a digital rectal exam for men, either (U.S. Preventive Services Task Force, 2012b).

Most adults with DS did not mention getting an influenza vaccination, which is recommended annually before flu season (Chicoine et al., 2010). Caregivers reported that most adults with DS received an influenza vaccination within the prior year. 
Only one adult with DS discussed health insurance, stating that she had recently changed from private insurance to Medicaid with no problem. The caregivers' responses to the question about health insurance for the adults with DS indicated that a slight majority had Medicare (51.7\%). Almost half were covered by private insurance $(48.3 \%)$, and almost half were covered by Medicaid (48.3\%). This percentage is lower than the $80 \%$ covered by Medicaid reported by Weir (Johnson, 2012). This difference is possibly attributable to the recruitment methods resulting in a more affluent sample. Because the question on the Health Questionnaire instructed the caregiver to select all that apply in regards to health insurance, this information does not indicate if this is primary insurance coverage or if it is secondary coverage. In fact, according to caregivers, most adults with DS were covered by two insurance providers, and some were covered by three.

Because adults with DS were asked open-ended questions about health-care utilization, the findings from self-report may be unreliable. However, data gained from caregivers on health-care utilization indicate that most health-care guidelines for adults with DS are being followed in this sample. Areas found to be lacking were vaccinations and screenings for cancer, which need to be addressed to improve the general health of adults with DS.

The fourth hypothesis at the outset of the current study was that self-reported health problems, health risks, and health-care utilization of adults with DS would be comparable to that from caregiver-report, but that caregiver-report would be more detailed. This was partially confirmed in that some variables of self-reported health problems, health risks, and health-care utilization were comparable to that of caregiverreport. However, not all variables were associated, and at the same time, there were significant differences amongst the means of some self- and caregiver-reported variables. The caregivers did provide more detail and specificity about health problems, health risks, and health-care utilization of adults with DS; therefore, this hypothesis was partially confirmed.

\section{Specific Aim Four}

The fourth specific aim was exploratory in nature and examined the association of the HRQOL of adults with DS to the health, adaptive behavior, and demographic characteristics of adults with DS. The following research question relates to this aim:

Research Question 4 What are the factors (health problems, health risks, healthcare utilization, adaptive behavior, and demographic characteristics) that are associated with the HRQOL of adults with DS as reported by:

a) adults with DS?

b) caregivers of adults with DS? 


\section{Adaptive behavior}

The scores from Vineland Adaptive Behavior Scales, Second Edition (VinelandII) indicated strengths and weaknesses in this sample of adults with DS. Of the three domains, the highest mean standard score demonstrating an area of strength was for the socialization domain and next the daily living skills domain, and the lowest score was for the communication domain. This lowest score on the communication domain is consistent with studies and guidelines related to communication impairment in DS (Brown, 1998; Fujiura \& Behrens, 2008; McGuire \& Chicoine, 2006). All mean domain scores fell into the low adaptive level ( $\leq 70$; Sparrow, Cicchetti, \& Balla, 2005). However, if the standard deviations are added to the means of the domain scores to get the highest scores achieved by this sample, the upper score of the socialization domain and the daily living skills domain are in the moderately low adaptive level (71-85; Sparrow et al., 2005). The communication domain mean with the standard deviation added approximates the moderately low level at 69.43.

The means of the coping socialization subdomain and the receptive communication subdomain were in the moderately low adaptive level (v-scale score of 10-12; Sparrow et al., 2005). Means of the play and leisure socialization subdomain and the interpersonal relationships socialization subdomain fell between moderately low and low adaptive level. The means of the remaining subdomains were in the low adaptive level (v-scale score $\leq 9$; Sparrow et al., 2005). This included all subdomains in the daily living skills domain, which was the domain with the second-highest standard score. The lowest subdomain $\mathrm{V}$-scale score was for the written communication subdomain at 7.27 $(\mathrm{SD}=2.67)$.

The mean of the overall adaptive behavior composite standard score (54.42) was in the low adaptive level. The score at the highest level of the range of adaptive behavior composite standard score calculated by adding the standard deviation to the mean was 70.33, which falls between low and moderately low.

Extant literature on adaptive behavior of adults with DS reports low adaptive behavior levels for all domains and subdomains (Adams \& Oliver, 2010; Di Nuovo \& Buono, 2011; Dressler, Perelli, Feucht, \& Bargagna, 2010; Witts \& Elders, 1998). However, all studies used the Vineland Adaptive Behavior Scales (VABS) which complicates comparison. According to Sparrow et al. (2005), when the Vineland-II was created, the VABS was considerably modified to increase the age-range, which was birth to 19 years old for the VABS (Sattler, 1992).

Dressler et al. (2010) reported low adaptive levels for 40 adults with DS over the age of 20 residing in Italy, however they reported age-equivalents rather than v-scale scores and standard scores, which makes direct comparison challenging. Another challenge was that Dressler et al. separated the adults with DS into age groups of 20-30 years old and over 30 years old. Dressler et al. found age equivalents for all subdomains, domains, and the adaptive behavior composite at 10 years old or lower. Furthermore, the adaptive behavior composite score was 7.75 (SD 3.24) years for 20-30 year olds and 6.38 
(SD 3.46) years for those over 30 years old. Comparatively, the age equivalents for the subdomains of the adults with DS in the current study were 12 years old or lower, with only the three communication subdomains falling below 10 years old (receptive $=9.63$ years old, expressive $=8.06$ years old, and written $=8.42$ years old). The Vineland-II does not yield age equivalents for the domain scores or the adaptive behavior composite. However, because the subdomain age equivalents measured by Dressler et al. were lower than the subdomain age equivalents in the current study, a parallel can be established because the lower subdomain age equivalents would impact domain score means measured by Dressler et al., and therefore the adaptive behavior composite score mean, which would, thus, be lower than the means of all scores for the current study.

The adaptive behavior component score and more than half of the domain and subdomain scores from the Vineland-II had significant positive correlations $(p<.05)$ with the caregiver-reported MCS only. Perhaps this occurred because the behaviors of adults with DS that were evaluated by the Vineland-II are observable behaviors that do not generally require physical ability, which would increase association with the PCS. Additionally, "adaptive behavior is defined by the expectations or standards of other people" (Sparrow et al., 2005, p. 6); therefore the caregivers could observe actions that make up the Vineland-II and are, therefore, aware of the impact that adaptive behavior has on the HRQOL of adults with DS. It is likely that an adult with DS is unable to conceptually define his/her performance of actions evaluated on the Vineland-II and may be unaware of his/her own adaptive behavior and the impact it has on his/her physical or mental HRQOL.

\section{Predictors of health-related quality of life}

Independent variables from the Demographic Form, health interview, Health Questionnaire, and Vineland-II that were found to have associations with the dependent variables of self-reported PCS, self-reported MCS, caregiver-reported PCS, and caregiver-reported MCS at a significance of .2 or less were entered into multiple regression analysis with the dependent variable to which it was correlated, and stepwise multiple regression models were created in each category from which the independent variable came (e.g., demographic characteristic, health problem, health risk, health-care utilization, Vineland-II subdomain score, Vineland-II domain score, and Vineland-II adaptive behavior component score). To then substantiate the predictive probability of these independent variables, a stepwise multiple regression model was created for each dependent variable with the variables from all categories found to be predictive in the preliminary models.

Approximately 29\% of the variance in the self-reported PCS can be explained by the race of the adult with DS along with caregiver-reported problems with teeth or gums and asthma of the adult with DS. The race of adults with DS was obtained from the Demographic Form and, because it is a nominal variable, it was coded as $1=$ American Indian/Alaskan-native, 2=Asian, 3=Black or African-American, 4=Hispanic, 5=NativeHawaiian/Pacific-islander, $6=$ White, and $7=$ other. The $\beta$ and $t$ statistic for this variable 
were positive in value. Because the sample in this study was primarily white, the race categories were collapsed to dichotomous variables $(0=$ white and $1=$ all other $)$. This produced a regression model with the same three variables in which $\mathrm{R}^{2}=.282(\mathrm{p}=.000)$, confirming the original finding. Further investigation is strongly recommended because of the limited diversity of the sample. Additionally, the occurrence of the adult with DS having asthma had a positive $\beta$ for an increase in self-reported PCS which is difficult to explain and warrants further investigation. The $\beta$ for the adult with DS having dental problems per caregiver-report is negative, which indicates that occurrence of dental problems reported by caregivers predicts a lower self-reported PCS. This is understandable given that dental problems can impair the perception of an individual's physical HRQOL.

Approximately $40 \%$ of the variance of self-reported MCS can be explained by self-reported other health problems and feeling sad or blue and caregiver-reported frequency of using the bathroom at night and allergies. All standardized $\beta$ s and $t$ statistics were negative, which indicate that occurrence of feeling sad or blue per selfreport and allergies per caregiver-report result in a lower self-reported MCS score. Additionally, an increase in the number of other health problems per self-report and the number of times the adult with DS gets up at night to use the bathroom per caregiverreport are predictive of lower self-reported MCS scores. All of these predictors are reasonable in that symptoms of depression can decrease mental HRQOL and the other three physical problems can be associated with mental sequelae, especially getting up at night frequently which would interrupt nocturnal sleeping habits.

Approximately 54\% of the variance of caregiver-reported PCS can be explained by the state of residence of the adult with DS and caregiver, self-reported resistance to health care and trouble walking, and caregiver-reported trouble walking and weight problems. The nominal variable, state of residence, was coded as 1=Alabama, $2=$ Georgia, $3=$ Mississippi, $4=$ Tennessee, and 5=Florida. The $\beta$ and $t$ statistic for this variable was positive in value. Because more than half of the participants were from Tennessee, regression was reanalyzed using a dichotomous variable for the state of residence $(0=$ Tennessee, $1=$ all other. Again, the original findings were confirmed by a regression model of the same variables in which the $\mathrm{R}^{2}=.521(\mathrm{p}=.000)$. The other $\beta$ s and $t$ statistics were negative, indicating that trouble walking per self- and caregiver-report, resistance to health care per self, and weight problems per caregiver-report negatively affect the caregiver-reported PCS. These findings are understandable because difficulty walking and weight problems tend to be observable, and resistance to seek healthcare is likely recognized by the caregiver. The caregiver's awareness of these difficulties increases the likelihood the caregiver's estimation of physical HRQOL of the adult with DS would be decreased.

Finally, approximately $39 \%$ of the variance of caregiver-reported MCS can be explained by the living arrangement of the adult with DS and caregiver-reported problems with appetite. The living arrangement is a nominal variable that had higher scores for increased independence. Therefore, because the $\beta$ and $t$ statistic were positive, this can be interpreted that the more independent the living arrangement of the adult with 
DS, the higher the HRQOL as reported by the caregiver. The last predictor, problems with appetite per caregiver, had a negative $\beta$ and $t$ statistic, indicating that greater problems predict a lower MCS. This finding deserves further exploration.

The Vineland-II scores which were associated with HRQOL component summary scores were included in preliminary regression models, but they did not remain in the regression models that best predicted self- and caregiver-reported PCS and MCS. The association is consistent with extant literature on adaptive behavior and quality of life (Brown, 1994), but the lack of a predictive relationship is potentially resulting from the small sample size in the current study. Because this specific aim was exploratory and the sample size of the study was not large enough to yield statistical power for this aim, further investigation to confirm or refute this finding is appropriate.

Finding associations of adaptive behavior measures with HRQOL measures partially confirms the fifth hypothesis made at the onset of the study that associations would be revealed between the HRQOL scores and the health problems, health risks, health-care utilization, adaptive behavior, and demographic characteristics of adults with DS. Additional support for this hypothesis occurred through the association of health problems, health risks, and health-care utilization with the HRQOL of adults with DS. However, due to the challenges that were presented through the quantification of qualitative data from interviews, it was impossible to associate health-care utilization adequately to confirm positive associations with proactive health care and negative associations with reactive health care as stated in the hypothesis.

\section{Strengths and Limitations}

The primary strength of this study was the successful recruitment of 60 dyads of adults with DS and their caregivers. This permitted achievement of statistical power to address research questions 1, 2, and 3 (Prescott, 1987).

Additionally, using the mixed-methods approach permitted interactions with the adults with DS. Because of this approach, more meaningful data were obtained, and the bias of yes/no questions found in research involving individuals with IDD was avoided (Finlay \& Lyons, 2001), providing further strength to the study.

A third strength of the study was the participation of close family members, mostly parents, of the adults with DS as caregivers. These individuals have more familiarity with the thoughts and feelings of the adults with DS than most support staff would have. The solid relationships represented in the study likely enhanced validity and quality to the data collected from caregivers.

A fourth strength is that the findings from this study support examining HRQOL using the LCP theoretical model. The paradigmatic principles for LCP were applicable to this study. Life-span development was evident when adults with DS discussed and demonstrated an understanding of health-problems or risks occurring during adulthood; 
agency was exhibited when adults with DS participated in interviews, completed verbal questionnaires, and discussed choosing activities that provide exercise and social interaction; time and place was exhibited by younger birth cohorts attending postsecondary education and by the state of residence (Tennessee) serving as a predictor of caregiver-reported PCS of adults with DS; timing was demonstrated by caregivers of adults with DS in their forties reporting memory-problems which may be symptomatic of earlier onset of dementia or AD than in adults without DS; and, as discussed previously, the principle of linked lives was supported by this research through the observed and reported relationships between adults with DS and their caregivers. The adults with DS reported transitions (e.g., death of a parent, getting a new job) which theoretically influence their trajectories. Finally, all factors in the hypothesized theoretical model (i.e., health problems, health risks, health-care utilization, and adaptive behavior), demonstrated some associations with the HRQOL of adults with DS in this study. The fifth hypothesis that LCP is relevant to the HRQOL of adults with DS can be supported.

The final strength of this study is the contribution of information about HRQOL of adults with DS to a field of research that is sparse. Findings from this study can inspire and support future investigations of the HRQOL of adults with DS.

Conversely, one of the most notable limitations of the study arose from the quantification of data from the interviews of adults with DS. Because the format of questions was different between those asked of the adults with DS and those asked of their caregivers, the limitation arose in the comparison of the quantified data from the interviews with the responses from the Health Questionnaire. Due to the inconsistency of question format, comparison of self-reported health problems, health risks, and healthcare utilization to that reported by caregivers was imprecise. Finally, open-ended questions during the interviews with adults with DS left the frequency and intensity of health problems undefined from the perspective of adults with DS.

The next limitation of the study is lack of a representative sample. The recruitment technique for this study was network or snowball sampling, which often results in a sample that is decidedly homogenous (Polit \& Beck, 2008). Efforts to avoid homogeneity would have extended the study and required additional resources. Additionally, the study was limited to four states in the southeastern U.S. Regional characteristics could prevent the results of the study from being generalizable to other regions of the U.S. or internationally. Despite this limitation, the study is noteworthy as this is the only known study to examine self-reported and caregiver-reported perspectives of HRQOL of adults with DS between adults with DS and their caregivers. Thus, this study addresses an important area of inquiry.

Another limitation is that the sample size was not large enough to achieve statistical power for research question 4 , relegating this to the status of an exploratory question. The investigator had neither the time nor the resources necessary to collect data from the large number of participants required to attain statistical power for this question. However, additional research will be possible in the future and important insights were gained that will inform the designs and approaches used in future studies. 
Considering that the occurrence of AD or dementia can begin when an adult with DS is 40 years old (Bosch, 2003; Kerins et al., 2008), another limitation is the uncertain reliability of self-report of the adults over the age of 40. Additionally, inclusion of data from two adults who had diagnoses of dementia and 16 others that had memory problems per caregiver-report may have hindered the reliability of this self-reported data. However, the investigator had a degree of confidence that most adults with DS over the age of 40 comprehended the questions and answered appropriately and consistently.

A theoretical limitation of this study was the cross-sectional design. Life Course Perspective is best applied to a longitudinal study, which was beyond the capabilities and resources of the investigator at the time of the study. It is an aspiration of the investigator to recruit the same participants in future research, which can include these findings as the starting time-point for a longitudinal design.

Finally, the SF-12v2 is perhaps not the ideal instrument for assessment of HRQOL of all adults with DS, because only $45 \%$ of the adults with DS in this study demonstrated veritable responses of 10 or more of the 12 questions based on investigator perception. Additionally, $27 \%$ of the adults with DS in this study did not reliably answer at least half of the questions. However, the discriminant validity was perfect for adults with DS, and the convergent validity was reasonable, so in lieu of a DS-specific tool, the SF-12v2 appears to sufficiently measure the HRQOL for this sample.

\section{Significance}

\section{Research implications}

As mentioned previously, this study is significant in that it investigated information with an understudied population. The HRQOL of adults with DS has not been examined, so the findings from this study will contribute to this field of knowledge. The instruments used in this study had not been reported in published research on adults with DS, so this study can help establish a baseline measurement for both the SF-12v2 and the Vineland-II for this population.

The exploratory research question needs further investigation with a larger sample. Research in this area could provide reliable information on the extent that demographic characteristics, health problems, health risks, health-care utilization, and adaptive behavior predict HRQOL of adults with DS.

Additionally, questions arose during the health interviews and analysis of the Health Questionnaires, such as indefinite findings about osteoporosis, pneumonia, and alcohol use, as well as the perception of depression in adults with DS. These uncertainties substantiate rationale for future research, perhaps with the use of electronic health records. 
The instruments utilized in this study were imperfect. There were concerns about comparing open-ended questions from the health interviews with yes/no questions from the Health Questionnaire. These challenges provide an opportunity to modify the methods and repeat the study to confirm, refute, or meliorate the findings. The challenge some adults with DS had comprehending the SF-12v2 can inspire formation of a different instrument or approach to measure the HRQOL of adults with DS.

There is also the prospect of approaching the participants in this study for participation in future research. By repeating the measurements of HRQOL, health problems, health risks, health-care utilization, and adaptive behavior with the same participants periodically, changes that occur during aging could be investigated.

Finally, there is justification for exploring an interventional study that includes adults with DS as participants in their health care and also involving their caregivers. By implementing interventions to improve health, the HRQOL of adults with DS could improve.

\section{Practice implications}

Based on the findings in this study, health-care providers can feel more confident interviewing adults with DS directly in the clinical setting. This not only recognizes the self-determination of the adults with DS, it provides valuable information that helps complete the provider's understanding of the health of an adult with DS. Empowering patients by including them in health-improvement plans and by investigating and incorporating their preferences and willingness to participate improves the potential for success of the plan (Ignatavicius, 2010). These findings can also inform health-care providers and caregivers that adults with DS place more emphasis on health problems that have manifestations that are not observable, while caregivers place more emphasis on health problems with visible physical manifestations.

Other implications for the practice of health-care providers are to ask about the health issues and risks that are problematic for adults with DS. While assessments for thyroid disorder, sleep apnea, and early dementia may seem theoretical in nature, the findings of this study support the importance of these assessments. Furthermore, healthcare providers may now recognize the need for assessments for other less common health problems and risks in the literature that were prevalent in this study, such as headaches, musculoskeletal problems, and alcohol consumption.

This study reveals areas where some adults with DS are not participating in recommended screenings and treatments, such as routine Pap smears, mammograms, and testicular exams. Because nurses are often in the role of advocates for patients (Ignatavicius, 2010) these data can provide nurses and other health-care providers with information to educate and encourage adults with DS on the importance of routine screening. 
Finally, the study inadvertently found that many adults with DS are afraid of health-care visits. This should encourage the health-care providers to make attempts to improve trust and relationships with adults with DS in the health-care environment which can lessen their fear.

\section{Theoretical implications}

Because the HRQOL of adults with DS supports the principles of LCP and the associations between HRQOL and health problems, health risks, health-care utilization, and adaptive behavior were demonstrated, this cross-sectional study can be an impetus for other cross-sectional studies on the HRQOL of different populations and other applicable concepts. Additionally, to further explore the trajectories of individuals from the LCP framework, a longitudinal study, as mentioned previously, could follow this sample and continue to investigate HRQOL, health problems, health risks, health-care utilization, and adaptive behavior attuned to elements and principles of LCP.

\section{Policy implications}

Support/advocacy organizations can use this study as evidence to educate and encourage adults with DS to have routine cancer screenings, cervical spine $\mathrm{x}$-rays, and vaccinations, since the findings of this study indentified a deficit in these areas of healthcare utilization. Enhanced networking between DS support/advocacy organizations would also be beneficial to individuals with DS who move from one area to another and to researchers wanting to contribute to the knowledge about individuals with DS.

\section{Conclusion}

Adults with DS are living longer and have new opportunities to become more involved in their communities. By having the ability to measure the HRQOL of adults with DS and identify factors that contribute to it, nurses and health-care providers can help adults with DS optimize their independence and self-determination by addressing their health problems, health-risks, health-care utilization, and adaptive behavior.

This study was the first to measure self-reported HRQOL, health problems, health risks, and health-care utilization of adults with DS and to compare it to that reported by caregivers. This study was also the first to explore factors that are associated with the HRQOL of adults with DS such as demographic characteristics, health problems, health risks, health-care utilization, and adaptive behavior. By accomplishing these aims, this study has contributed to both nursing science and the science related to the HRQOL of adults with DS. Although all hypotheses were not confirmed, the findings of the study provide a platform upon which to continue investigation. 
It was an objective of the investigator to acknowledge and respect the selfdetermination and dignity of adults with DS, a goal consistent with the LCP principle of agency. This goal was implemented by asking adults with DS if the caregiver could provide information about them during the consent process; by asking open-ended questions about health problems, health risks, and health-care utilization during the interviews; and by assessing HRQOL by directly asking the adults with DS questions to measure this subjective phenomenon.

This study revealed that adults with DS have above-average HRQOL compared to the rest of the United States based on self-report and caregiver-report. Findings suggested that self-report and caregiver-report are not always in complete agreement, but that there were many associations between the two. Finally, findings indicated several contributing factors to HRQOL of adults with DS that should undergo further investigation. This provides a hopeful mission to nursing science to strive to improve HRQOL of adults with DS through interventions, respect, and inclusion of adults with DS in the health-care decision-making process. 


\section{LIST OF REFERENCES}

Adams, D., \& Oliver, C. (2010). The relationship between acquired impairments of executive function and behavior change in adults with Down syndrome. Journal of Intellectual Disability Research, 54(5), 393-405. doi:10.1111/j.13652788.2010.01271.x

Ailey, S. H., \& Melich-Munyan, T. (2010). Mental and behavioral health disorders in individuals with intellectual and developmental disabilities. In C. L. Betz \& W. M. Nehring (Eds.), Nursing care for individuals with intellectual and developmental disabilities: An integrated approach (pp. 257-276). Baltimore: Paul H. Brookes Publishing Co., Inc.

Alexander, G. E., Saunders, A. M., Szczepanik, J., Strassburger, T. L., Pietrini, P., Dani, A., ... Schapiro, M. B. (1997). Relation of age and apolipoprotein E to cognitive function in Down syndrome adults. NeuroReport, 8, 1835-1840.

Alford, C., \& Nurudeen, S. (2013). Physiology of reproduction in women. In A. H. DeCherney, N. Laufer, L. Nathan, \& A. S. Roman (Eds.), CURRENT Diagnosis \& Treatment: Obstetrics \& Gynecology (11th ed., pp. 97-118). New York: McGraw-Hill Companies, Inc.

Allison, P. J., Hennequin, M., \& Faulks, D. (2000). Dental care access among individuals with Down syndrome in France. Special Care in Dentistry, 20, 28-34.

Anderson, K. N., Anderson, L. E., \& Glanze, W. D. (1998). Mosby's medical, nursing, \& allied health dictionary (5th ed.). St. Louis, MO: Mosby-Year Book, Inc.

Anwar, A. J., Walker, J. D., \& Frier, B. M. (1998). Type 1 diabetes mellitus and Down's syndrome: Prevalence, management and diabetic complications. Diabetic Medicine, 15, 160-163.

Arlt, S., Hornung, J., Eichenlaub, M., Jahn, H., Bullinger, M., \& Petersen, C. (2008). The patient with dementia, the caregiver and the doctor: Cognition, depression and quality of life from three perspectives. International Journal of Geriatric Psychiatry, 23, 604-610. doi:10.1002/gps.1946

Ben-Shlomo, Y., \& Kuh, D. (2002). A life course approach to chronic disease epidemiology: Conceptual models, empirical challenges and interdisciplinary perspectives. International Journal of Epidemiology, 31, 285-293.

Bertoli, M., Biasini, G., Calignano, M. T., Celani, G., De Grossi, G., Digilio, M. C., . . . Zuccala, G. (2011). Needs and challenges of daily life for people with Down syndrome residing in the city of Rome, Italy. Journal of Intellectual Disability Research, 55(8), 801-820. doi:10.1111/j.1365-2788.2011.01432.x 
Betz, C. L., \& Jones, M. W. (2010). Nursing care approaches: Clinical and practical considerations. In C. L. Betz \& W. M. Nehring (Eds.), Nursing care for individuals with intellectual and developmental disabilities: An integrated approach (pp. 147-171). Baltimore: Paul H. Brookes Publishing Co., Inc.

Birenbaum, A., \& Cohen, H. J. (2006). Utilization, cost, and financing. In I. L. Rubin \& A. C. Crocker (Eds.), Medical care for children and adults with developmental disabilities (2nd ed., pp. 673-687). Baltimore, MD: Paul H. Brookes Publishing Co., Inc.

Bittles, A. H. \& Glasson, E. J. (2004). Clinical, social, and ethical implications of changing life expectancy in Down syndrome. Developmental Medicine \& Child Neurology, 46, 282-286. doi: 10.1017/S0012162204000441

Black, B. P., Holditch-Davis, D., \& Miles, M. S. (2009). Life Course Theory as a framework to examine becoming a mother of a medically fragile preterm infant. Research in Nursing and Health, 32, 38-49. doi:10.1002/nur.20298

Bosch, J. J. (2003). Health maintenance throughout the life span for individuals with Down syndrome. Journal of the American Academy of Nurse Practitioners, 15, 5 17. doi: 10.1111/j.1745-7599.2003.tb00248.x

Brown, R. I. (1994). Down syndrome and quality of life: Some challenges for future practice. Down Syndrome Research and Practice, 2, 19-30.

Brown, R. I. (1998). The effects of quality of life models on the development of research and practice in the field of Down syndrome. Down Syndrome Research and Practice, 5, 39-42.

Brown, R. I., MacAdam-Crisp, J., Wang, M., \& Iarocci, G. (2006). Family quality of life when there is a child with a developmental disability. Journal of Policy and Practice in Intellectual Disabilities, 3(4), 238-245. doi:10.1111/j.17411130.2006.00085.x

Brown, R., Taylor, J., \& Matthews, B. (2001). Quality of life: Ageing and Down syndrome. Down Syndrome Research and Practice, 6(3), 111-116.

Buckley, S. J. (2001). Reading and writing for individuals with Down syndrome: An overview. Down syndrome issues and information. Retrieved from http://www.down-syndrome.org/information/reading/overview/

Butterworth, J., Smith, F. A., Hall, A. C., Migliore, A., Winsor, J., Domin, D., \& Timmons, J. (2012). StateData: The national report on employment services and outcomes. Boston, MA: University of Massachusetts Boston, Institute for Community Inclusion. 
Byrne, A., MacDonald, J., \& Buckley, S. (2002). Reading, language and memory skills: A comparative longitudinal study of children with Down syndrome and their mainstream peers. British Journal of Educational Psychology, 72, 513-529.

Caldwell, J. (2008). Health and access to health care of female family caregivers of adults with developmental disabilities. Journal of Disability Policy Studies, 19(2), 6879. doi:10.1177/1044207308316093

Cheak-Zamora, N. C., Wyrwich, K. W., \& McBride, T. D. (2009). Reliability and validity of the SF-12v2 in the medical expenditure panel survey. Quality of Life Research, 18, 727-735. doi:10.1007/s11136-009-9483-1

Chicoine, B., McGuire, D., Bilodeau, J., \& Dominiak, E. (2010). Health issues for adults with Down syndrome. Adult Down Syndrome Center, Lutheran General Hospital. Retrieved from http://www.advocatehealth.com/luth/documents/downsyndrome/health $\% 20 \mathrm{ds} \% 20$ handout.pdf

Cohen, W. I. (Ed.) (1999). Health care guidelines for individuals with Down syndrome. Down Syndrome Quarterly, 4(3), 1-15.

Community-University Partnership for the Study of Children, Youth, and Families. (2011). Review of the Vineland Adaptive Behavior Scales-Second Edition (Vineland-II). Edmonton, Alberta, Canada.

Cooper, S.-A., Smiley, E., Jackson, A., Finlayson, J., Allan, L., Mantry, D., \& Morrison, J. (2009). Adults with intellectual disabilities: Prevalence, incidence and remission of aggressive behaviour and related factors. Journal of Intellectual Disability Research, 53(3), 217-232. doi:10.1111/j.1365-2788.2008.01127.x

Creswell, J. W., \& Plano Clark, V. L. (2011). Designing and conducting mixed methods research ( ${ }^{\text {nd }}$ ed.). Los Angeles, CA: Sage Publications, Inc.

Crocker, A. C. (2006a). Down syndrome. In I. L. Rubin \& A. C. Crocker (Eds.), Medical care for children and adults with developmental disabilities (2nd ed., pp. 178181). Baltimore, MD: Paul H. Brookes Publishing Co., Inc.

Crocker, A. C. (2006b). The developmental disabilities. In I. L. Rubin \& A. C. Crocker (Eds.), Medical care for children and adults with developmental disabilities (2nd ed., pp. 15-22). Baltimore, MD: Paul H. Brookes Publishing Co., Inc.

Crocker, A. C. (2006c). The spectrum of medical care. In I. L. Rubin \& A. C. Crocker (Eds.), Medical care for children and adults with developmental disabilities (2nd ed., pp. 23-32). Baltimore, MD: Paul H. Brookes Publishing Co., Inc. 
Crowther-Radulewicz, C. L., \& McCance, K. L. (2010). Alterations of musculoskeletal function. In K. L. McCance \& S. E. Huether (Eds.), Pathophysiology: The biologic basis for disease in adults and children (6th ed., pp. 1568-1617). Maryland Heights, MO: Mosby, Inc.

Cummins, R. A. (1997). Self-rated quality of life scales for people with an intellectual disability: A review. Journal of Applied Research in Intellectual Disabilities, 10(3), 199-216.

Dalton, A. J., \& McVilly, K. R. (2004). Ethics guidelines for international, multicenter research involving people with intellectual disabilities. Journal of Policy and Practice in Intellectual Disabilities, 1(2) 57-70.

Developmental Disabilities Assistance and Bill of Rights Act of 2000. 42 U.S.C. $§ 5801$ et seq.

Di Nuovo, S., \& Buono, S. (2011). Behavioral phenotypes of genetic syndromes with intellectual disability: Comparison of adaptive profiles. Psychiatry Research, 189, 440-445. doi:10.1016/j.psychres.2011.03.015

Draper, P., \& Thompson, D. R. (2001). The quality of life: A concept for research and practice. Nursing Times Research, 6, 648-657.

Dressler, A., Perelli, V., Feucht, M., and Bargagna, S. (2010). Adaptive behavior in Down syndrome: A cross-sectional study from childhood to adulthood. Wiener klinische Wochenschrift, 122, 673-680. doi:10.1007/s00508-010-1504-0

Elder, G. H., \& Giele, J. Z. (2009). Life course studies: An evolving field. In G. H. Elder \& J. Z. Giele (Eds.), The craft of life course research (pp. 1-24). New York: Guilford Press.

Elder, G. H., Johnson, M. K., \& Crosnoe, R. (2003). The emergence and development of life course theory. In J. T. Mortimer \& M. J. Shanahan (Eds.), Handbook of the life course (pp. 3-22). New York: Kluwer Academic/Plenum.

Esbensen, A. J. (2010). Health conditions associated with aging and end of life of adults with Down syndrome. International Review of Research in Mental Retardation, 39(C), 107-126. doi:10.1016/S0074-7750(10)39004-5

Esbensen, A. J., Bishop, S., Seltzer, M. M., Greenberg, J. S, \& Taylor, J. L. (2010). Comparisons between individuals with autism spectrum disorders and individuals with Down syndrome in adulthood. American Journal on Intellectual and Developmental Disabilities, 115(4), 277-290. doi:10.1352/1944-7558-115.4.277 
Esbensen, A. J., Seltzer, M. M., \& Greenberg, J. S. (2007). Factors predicting mortality in midlife adults with and without Down syndrome living with family. Journal of Intellectual Disability Research, 51(12), 1039-1050. doi: 10.1111/j.13652788.2007.01006.x

Esbensen, A. J., Seltzer, M. M., \& Krauss, M. W. (2008). Stability and change in health, functional abilities, and behavior problems among adults with and without Down syndrome. American Journal of Mental Retardation, 113(4), 263-277.

Estores, D. S. (2014). Symptom predictability in gastroesophageal reflux disease and role of proton pump inhibitor test. Gastroenterology Clinics of North America, 43, 27 38. doi:10.1016/j.gtc.2013.11.002

Ethen, M. K., \& Canfield, M. A. (2002). Impact of including elective pregnancy terminations before 20 weeks gestation on birth defect rates. Teratology, 66(Supp1 1), S32-S35.

Feng, L., Scherer, S. C., Tan, B. Y., Chan, G., Fong, N. P., \& Ng, T. P. (2010). Comorbid cognitive impairment and depression is a significant predictor of poor outcomes in hip fracture rehabilitation. International Psychogeriatrics, 22(2), 246-253. doi:10.1017/S1041610209991487

Finlay, W. M. L., \& Lyons, E. (2001). Methodological issues in interviewing and using self-report questionnaires with people with mental retardation. Psychological Assessment 13(3), 319-335.

Finlayson, J., Morrison, J., Jackson, A., Mantry, D., \& Cooper, S.-A. (2010). Injuries, falls and accidents among adults with intellectual disabilities: Prospective cohort study. Journal of Intellectual Disability Research, 54(11), 966-980. doi: 10.1111/j.1365-2788.2010.01319.x

Fujiura, G. T. (2012). Self reported health of people with intellectual disabilities. Intellectual and Developmental Disabilities, 50(4), 352-369. doi:10.1352/19349556-50.4.352

Fujiura, G. T., \& Behrens, C. (2008). Health status measurement of persons with intellectual disabilities: Development and testing of self report health related quality of life. Retrieved from http://www.rrtcadd.org/Research/Health\%20Status/Info.html

Fujiura, G. T., \& Behrens, C. (2011, May). Health status measurement of persons with intellectual disabilities: Development and testing of self report health related quality of life. Paper presented at the meeting of Health Frontier for Intellectual Disabilities, Bethesda, MD. Retrieved from http://www.rrtcadd.org/resources/Resources/Publications/Policy/Service/Briefs\&-Reports/RRTC-SOS-2011-Proceedings.pdf 
Galley, R. (2005). Medical management of the adult patient with Down syndrome. Journal of the American Academy of Physician Assistants, 18(4), 45-52.

Gandek, B., Ware, J. E., Aaronson, N. K., Apolone, G., Bjorner, J. B., Brazier, J. E., .. . Sullivan, M. (1998). Cross-validation of item selection and scoring for the SF-12 Health Survey in nine countries: Results from the IQOLA Project. Journal of Clinical Epidemiology, 51(11), 1171-1178.

Giele, J. Z., \& Elder, G. H. (1998). Life course research: Development of a field. In J. Z. Giele \& G. H. Elder (Eds.), Methods of life course research: Qualitative and quantitative approaches (pp. 5-27). Thousand Oaks, CA: Sage Publications, Inc.

Graff, J. C. (2010). Psychosocial issues. In C. L. Betz \& W. M. Nehring (Eds.), Nursing care for individuals with intellectual and developmental disabilities: An integrated approach (pp. 109-130). Baltimore: Paul H. Brookes Publishing Co., Inc.

Graff, J. C., Engle, V. F., \& Pruett, J. (2003, November). Health disparities of custodial and non-custodial black grandparents caring for grandchildren. Poster session presented at the $56^{\text {th }}$ annual meeting of the Gerontological Society of America, San Diego, CA.

Grant, G., Nolan, M., \& Keady, J. (2003). Supporting families over the life course: Mapping temporality. Journal of Intellectual Disability Research, 47(Pt 4-5), 342351. doi:10.1046/j.1365-2788-2003-00495.x

Guyatt, G. H., Feeny, D. H., \& Patrick, D. L. (1993). Measuring health-related quality of life. Annals of Internal Medicine, 118, 622-629.

Halfon, N., \& Hochstein, M. (2002). Life Course Health Development: An integrated framework for developing health, policy, and research. The Milbank Quarterly, $80(3), 433-479$

Havercamp, S. M., Scandlin, D., \& Roth, M. (2004). Health disparities among adults with developmental disabilities, adults with other disabilities, and adults not reporting disability in North Carolina. Public Health Reports, 119, 418-426.

Hedov, G., Anneren, G., \& Wikblad, K. (2000). Self-perceived health in Swedish parents of children with Down's syndrome. Quality of Life Research, 9, 415-422.

Heller, T., Caldwell, J., \& Factor, A. (2007). Aging family caregivers: Policies and practices. Mental Retardation and Developmental Disabilities Research Reviews, 13(2), 136-142. doi:10.1002/mrdd.20138 
Hewitt, L. E., Hinkle, A. S., \& Miccio, A. W. (2005). Intervention to improve expressive grammar for adults with Down syndrome. Communication Disorders Quarterly, 26(3), 144-155. doi:10.1177/15257401050260030301

Hodapp, R. M., \& Urbano, R. C. (2007). Adult siblings of individuals with Down syndrome versus with autism: Findings from a large-scale US survey. Journal of Intellectual Disability Research, 51(12), 1018-1029. doi:10.1111/j.13652788.2007.00994.x

Huether, S. E. (2010). Pain, temperature regulation, sleep, and sensory function. In K. L. McCance \& S. E. Huether (Eds.), Pathophysiology: The biologic basis for disease in adults and children (6th ed., pp. 481-524). Maryland Heights, MO: Mosby, Inc.

Ignatavicius, D. D. (2010). Introduction to medical-surgical nursing. In D. D. Ignatavicius \& M. L. Workman (Eds.), Medical-surgical nursing: Patientcentered collaborative care ( $6^{\text {th }}$ ed., pp. 2-7). St. Louis, MO: Saunders, an imprint of Elsevier, Inc.

Individuals with Disabilities Education Act of 1975, 20 U.S.C. $§ 1400$ et seq.

Johnson, B. (2012, July 23). Doctors staying in Medicaid a 'huge concern' for Down syndrome community. PJ Media. Retrieved from http://pjmedia.com/blog/doctors-staying-in-medicaid-a-huge-concern-for-downsyndrome-community/

Jones, J., Dagnan, D., \& Ruddick, L. (1997). A pilot study of the use of the SF-36 to assess health status of adults with learning disabilities living in small community based homes. British Journal of Developmental Disabilities, 84, 27-35.

Jones, K. L. (1997). Smith's recognizable patterns of human malformation $\left(^{\text {th }} \mathrm{ed}\right.$.). Philadelphia: W.B. Saunders Company.

Jonsson, L., Andreasen, N., Kilander, L., Soininen, H., Waldemar, G., Nygaard, H., .. . Wimo, A. (2006). Patient- and proxy-reported utility in Alzheimer disease using the EuroQoL. Alzheimer Disease Associated Disorders, 20, 49-55.

Jorde, L. B. (2010). Genes and genetic diseases. In K. L. McCance \& S. E. Huether (Eds.), Pathophysiology: The biologic basis for disease in adults and children (6th ed., 126-163). Maryland Heights, MO: Mosby, Inc.

Kaye, P. L., Fiske, J., Bower, E. J., Newton, J. T., \& Fenlon, M. (2005). Views and experiences of parents and siblings of adults with Down syndrome regarding oral healthcare: A qualitative and quantitative study. British Dental Journal, 198(9), 571-578. doi:10.1038/sj.bdj.4812305 
Keller, S. D., Bayliss, M. S., Ware, J. E., Jr., Hsu, M.-A, Damiano, A. M., \& Goss, T. F. (1997). Comparison of responses to SF-36 Health Survey questions with oneweek and four-week recall periods. Health Services Research, 32(3), 367-384.

Kerins, G., Petrovic, K., Bruder, M. B., \& Gruman, C. (2008). Medical conditions and medication use in adults with Down syndrome: A descriptive analysis. Down Syndrome Research and Practice, 12(2), 141-147. doi: 10.3104/reports.2009

Kerner, D. N., Patterson, T. L., Grant, I., \& Kaplan, R. M. (1998). Validity of the Quality of Well-Being Scale for patients with Alzheimer's Disease. Journal of Aging and Health, 10, 44-61.

Krahn, G. L., Hammond, L., \& Turner, A. (2006). A cascade of disparities: Health and health care access for people with intellectual disabilities. Mental Retardation and Developmental Disabilities Research Reviews, 12, 70-82. doi:10.1002/mrdd.20098

Lefter, S., Costello, D. J., McNamara, B., \& Sweeny, B. (2011). Clinical and EEG features of seizures in adults with Down syndrome. Journal of Clinical Neurophysiology, 28(5), 469-473. doi:10.1097/WNP.0b013e318230da76

Leonard, S., Bower, C., Petterson, B., \& Leonard, H. (1999). Medical aspects of schoolaged children with Down syndrome. Developmental Medicine and Child Neurology, 41(10), 683-688.

Leonard, S., Msall, M., Bower, C., Tremont, M., \& Leonard, H. (2002). Functional status of school-aged children with Down syndrome. Journal of Paediatrics and Child Health, 38(2), 160-165. doi: 10.1046/j.1440-1754.2002.00736.x

Lloyd, V., Gatherer, A., \& Kalsy, S. (2006). Conducting qualitative interview research with people with expressive language difficulties. Qualitative Health Research, 16(10), 1386-1404. doi:10.1177/1049732306293846

Lockhart, P. B., Hanson, N. B., Ristic, H., Menezes, A. R., \& Baddour, L. (2013). Acceptance among and impact on dental practitioners and patients of American Heart Association recommendations for antibiotic prophylaxis. The Journal of the American Dental Association, 144(9), 1030-1035. doi: 10.14219/jada.archive.2013.0230

Lott, I. T. (2012). Neurological phenotypes for Down syndrome across the life span. Progress in Brain Research, 197, 101-121. doi:10.1016/B978-0-444-542991.00006-6

Lott, I. T., Doran, E., Nguyen, V. Q., Tournay, A., Movsesyan, N., Gillen, D. L. (2012). Down syndrome and dementia: Seizures and cognitive decline. Journal of Alzheimer's Disease, 29(1), 177-185. doi:10.3233/JAD-2012-111613 
Mahy, J., Shields, N., Taylor, N. F., \& Dodd, K. J. (2010). Identifying facilitators and barriers to physical activity for adults with Down syndrome. Journal of Intellectual Disability Research, 54(9), 795-805. doi: 10.1111/j.13652788.2010.01308.x

Mai, C. T., Kucik, J. E., Isenburg, J., Feldkamp, M. L., Marengo, L. K., Bugenske, E. M., ... Kirby, R. S. (2013). Selected birth defects data from population-based birth defects surveillance programs in the United States, 2006-2010: Featuring trisomy conditions. Birth Defects Research. Part A, Clinical and Molecular Teratology, 97(11), 709-725. doi:10.1002/bdra.23198

McCarron, M., Gill, M., McCallion, P., \& Begley, C. (2005). Health co-morbidities in ageing persons with Down syndrome and Alzheimer's dementia. Journal of Intellectual Disability Research, 49(7), 560-566. doi: 10.1111/j.13652788.2005.00704.x

McCarron, M., McCallion, P., Fahey-McCarthy, E., Connaire, K., \& Dunn-Lane, J. (2010). Supporting persons with Down syndrome and advanced dementia: Challenges and care concerns. Dementia, 9(2), 285-298. doi: $10.1177 / 1471301209354025$

McGillicuddy, N. B. (2006). A review of substance use research among those with mental retardation. Mental Retardation and Developmental Disabilities, 12, 4147. doi:10.1002/mrdd.20092

McGrath, R. J., Stransky, M. L., Cooley, W. C., \& Moeschler, J. B. (2011). National profile of children with Down syndrome: Disease burden, access to care, and family impact. The Journal of Pediatrics, 159(4), 535-540.e2. doi: 10.1016/j.jpeds.2011.04.019

McGuire, D., \& Chicoine, B. (2006). Mental wellness in adults with Down syndrome: A guide to emotional and behavioral strengths and challenges. Bethesda, MD: Woodbine House, Inc.

Medicare.gov. (n.d.). The Affordable Care Act \& Medicare. Retrieved from http://www.medicare.gov/about-us/affordable-care-act/affordable-care-act.html

Megarbane, A., Ravel, A., Mircher, C., Sturtz, F., Grattau, Y., Rethore, M.-O., . . . Mobley, W. C. (2009). The 50th anniversary of the discovery of trisomy 21: The past, present, and future of research and treatment of Down syndrome. Genetics in Medicine, 11(9), 611-616. doi:10.1097/GIM.0b013e3181b2e34c

Natoli, J. L., Ackerman, D. L., McDermott, S., \& Edwards, J. G. (2012). Prenatal diagnosis of Down syndrome: A systematic review of termination rates (19952011). Prenatal Diagnosis, 32(2), 142-153. doi:10.1002/pd.2910 
Nehring, W. M., \& Betz, C. L. (2010). Down syndrome. In C. L. Betz \& W. M. Nehring (Eds.), Nursing care for individuals with intellectual and developmental disabilities: An integrated approach (pp. 211-234). Baltimore: Paul H. Brookes Publishing Co., Inc.

Palmer, F. B. (2010, Sept.). Aspects of normal and abnormal child development: A lifecourse perspective. In R.J. Roberts (Coordinator), Development and Disabilities: A Lifecourse Perspective. Conducted at the meeting of Leadership in Neurodevelopmental and Related Disabilities Trainee Minor Interdisciplinary Leadership Training, Memphis, TN.

Patient Protection and Affordable Care Act of 2010, 42 U.S.C. $§ 18001$ et seq.

Polit, D. F., \& Beck, C. T. (2008). Nursing research: Generating and assessing evidence for nursing practice (8th ed.). Philadelphia, PA: Lippincott Williams \& Wilkins.

Potter, P. A. (2009). Implementing nursing care. In P. A. Potter \& A. G. Perry (Eds.), Fundamentals of nursing (7th ed., pp. 278-289). St. Louis, MO: Mosby, Inc.

Prescott, P. A. (1987). Multiple regression analysis with small samples: Cautions and suggestions. Nursing Research, 36(2), 130-133.

Putney, N. M., \& Bengtson, V. L. (2003). Intergenerational relations in changing times. In J. T. Mortimer \& M. J. Shanahan (Eds.), Handbook of the life course (pp. 149164). New York: Kluwer Academic/Plenum.

Quinn, C., Dunbar, S. B., Clark, P. C., \& Strickland, O. L. (2010). Challenges and strategies of dyad research: Cardiovascular examples. Applied Nursing Research, 23, e15-e20. doi:10.1016/j.apnr.2008.10.001

Radbruch, L., Sabatowski, R., Loick, G., Jonen-Thielemann, I., Kasper, M., Gondek, B., . . Thielemann, I. (2000). Cognitive impairment and its influence on pain and symptom assessment in a palliative care unit: Development of a minimal documentation system. Palliative Medicine, 14(4), 266-276.

Roizen, N. J., Magyar, C. I., Kuschner, E. S., Sulkes, S. B., Druschel, C., van Wijngaarden, E., .. . Hyman, S. L. (2013). A community cross-sectional survey of medical problems in 440 children with Down syndrome in New York state. The Journal of Pediatrics. Advance online publication. doi:10.1016/j.jpeds.2013.11.032

Ruddick, L., \& Oliver, C. (2005). The development of a health status measure for selfreport by people with intellectual disabilities. Journal of Applied Research in Intellectual Disabilities, 18, 143-150. 
Sachs, G. A., Carter, R., Holtz, L. R., Smith, F., Stump, T. E., Tu, W., \& Callahan, C. M. (2011). Cognitive impairment: An independent predictor of excess mortality. Annals of Internal Medicine, 155(5), 300-308. doi:10.7326/0003-4819-155-5201109060-00007

Sandelowski, M., Voils, C. I., \& Knafl, G. (2009). On quantitizing. Journal of Mixed Methods Research, 3(3), 208-222. doi:10.1177/1558689809334210

Saris-Baglama, R. N., Dewey, C. J., Chisholm, G. B., Plumb, E., King, J., Rasicot, P., . . . Ware, J. E., Jr., (2011). QualityMetric Health Outcomes ${ }^{\text {TM }}$ Scoring Software 4.5 User's Guide. Lincoln, R.I.: QualityMetric Inc.

Sattler, J. M. (1992). Assessment of children (Rev. and updated 3rd ed.). San Diego: Author.

Schalock, R. L., Borthwick-Duffy, S. A., Bradley, V. J., Buntinx, W. H. E., Coulter, D. L., Craig, E. M., . . . Yeager, M. H. (2010). Intellectual disability: Definition, classification, and systems of supports: The AAIDD Ad Hoc Committee on Terminology and Classification (11th ed.). Washington, DC: American Association on Intellectual and Developmental Disabilities.

Schieve, L. A., Boulet, S. L., Boyle, C., Rasmussen, S. A., \& Schendel, D. (2009). Health of children 3 to 17 years of age with Down syndrome in the 1997-2005 National Health Interview Survey. Pediatrics, 123(2), e253-e260. doi:10.1542/peds.20081440

Seltzer, M. M., Floyd, F. J., Greenberg, J. S., Hong, J., Taylor, J. L., \& Doescher, H. (2009). Factors predictive of midlife occupational attainment and psychological functioning in adults with mild intellectual deficits. American Journal on Intellectual and Developmental Disabilities, 114(2), 128-143. doi:10.1352/2009.114.128-143

Seltzer, M. M., Krauss, M. W., Hong, J., \& Orsmond, G. I. (2001). Continuity or discontinuity of family involvement following residential transitions of adults who have mental retardation. Mental Retardation, 39(3), 181-194.

Servais, L. (2006). Sexual health care in persons with intellectual disabilities. Mental Retardation and Developmental Disabilities Research Reviews, 12, 48-56. doi:10.1002/mrdd.20093

Service, K. P. \& Hahn, J. E. (2003). Issues in aging: The role of the nurse in the care of older people with intellectual and developmental disabilities. Nursing Clinics of North America, 38, 291-312. doi:10.1016/S0029-6465(02)00055-5 
Seymour, D. G., Ball, A. E., Russell, E. M., Primrose, W. R., Garratt, A. M., \& Crawford, J. R. (2001). Problems in using health survey questionnaire in older patients with physical disabilities: The reliability and validity of the SF-36 and the effect of cognitive impairment. Journal of Evaluation in Clinical Practice, 7(4), 411-418.

Shimizu, R., Engel, J. D., \& Yamamoto, M. (2008). GATA1-related leukaemias. Nature Reviews Cancer, 8(4), 279-287. doi: 10.1038/nrc2348

Smith, D. S. (2001). Health care management of adults with Down syndrome. American Family Physician, 64(6), 1031-1038.

Smith, K., \& Savage, T. A. (2010). Policies, legislation, and ethical/legal issues. In C. L. Betz \& W. M. Nehring (Eds.), Nursing care for individuals with intellectual and developmental disabilities: An integrated approach (pp. 355-370). Baltimore: Paul H. Brookes Publishing Co., Inc.

Sparrow, S. S., Cicchetti, D. V., \& Balla, D. A. (2005). Vineland Adaptive Behavior Scales: Second Edition: Survey forms manual. Minneapolis, MN: NCS Pearson, Inc.

Stade, B. C., Stevens, B., Ungar, W. J., Beyene, J., \& Koren, G. (2006). Health-related quality of life of Canadian children and youth prenatally exposed to alcohol. Health and Quality of Life Outcomes, 4(81). doi:10.1186/1477-7525-4-81

Stebbins, L. F. (2001). Work and family in America: A reference handbook. Santa Barbara, CA: ABC-CLIO.

Taylor, J. L., \& Seltzer, M. M. (2012). Developing a vocational index for adults with autism spectrum disorders. Journal of Autism and Developmental Disorders, 42(12), 2669-2679. doi: 10.1007/s10803-012-1524-x

Thomas, K., Bourke, J., Girdler, S., Bebbington, A., Jacoby, P., \& Leonard, H. (2011). Variation over time in medical conditions and health service utilization of children with Down syndrome. The Journal of Pediatrics, 158(2), 194-200. doi:10.1016/j.jpeds.2010.08.045

Thorne, S., \& Paterson, B. (1998). Shifting images of chronic illness. Image: The Journal of Nursing Scholarship, 30(2), 173-179.

Turk, M. A., Geremski, C. A., Rosenbaum, P. F., \& Weber, R. J. (1997). The health status of women with cerebral palsy. Archives of Physical Medicine and Rehabilitation, 78(12 supp 5), S10-S17. 
U.S. Department of Education, Office of Special Education and Rehabilitative Services. (2010). Thirty-five years of progress in educating children with disabilities through IDEA. Washington, DC: Government Printing Office.

U.S. Preventive Services Task Force. (2012a). Screening for Prostate Cancer. Retrieved from http://www.uspreventiveservicestaskforce.org/prostatecancerscreening.htm

U.S. Preventive Services Task Force. (2012b). Understanding Task Force Recommendations: Screening for Prostate Cancer. Retrieved from www.uspreventiveservicestaskforce.org/prostatecancerscreening/prostatecancerfa ct.pdf

University of Tennessee Health Science Center Institutional Review Board. (2010). Standard Operating Procedure Number: IRB 005 UTHSC IRB Informed Consent, Version Number 006. Retrieved from http://www.uthsc.edu/research/research_compliance/IRB/policies.php

van Gameren-Oosterom, H. B. M., Fekkes, M., Buitendijk, S. E., Mohangoo, A. D., Bruil, J., \& Van Wouwe, J. P. (2011). Development, problem behavior, and quality of life in a population based sample of eight-year-old-children with Down syndrome. PLoS ONE 6(7): e21879. doi:10.1371/journal.pone.0021879

Verstegen, R. H. J., van Gameren-Oosterom, H. B. M., Fekkes, M., Dusseldorp, E., de Vries, E., \& van Wouwe, J. P. (2013). Significant impact of recurrent respiratory tract infections in children with Down syndrome. Child: Care, Health and Development, 39(6), 801-809. doi:10.1111/j.1365-2214.2012.01413.x

Vicari, S., Bellucci, S., \& Carlesimo, G. A. (2000). Implicit and explicit memory: A functional dissociation in persons with Down syndrome. Neuropsychologia, 38 240-251.

Virji-Babul, N., Eichmann, A., Kisly, D., Down, J., \& Haslam, R. H. A. (2007). Use of health care guidelines in patients with Down syndrome by family physicians across Canada. Paediatrics \& Child Health, 12(3), 179-183.

Vogels, T., Verrips, G. H. W., Verloove-Vanhorick, S. P., Fekkes, M., Kamphuis, R. P., Koopman, H. M., . . . Wit, J. M. (1998). Measuring health-related quality of life in children: The development of the TACQOL parent form. Quality of Life Research, 7(5), 457-465.

Ware, J. E., Jr., Kosinski, M., Turner-Bowker, D. M., Sundaram, M., Gandek, B., \& Maruish, M. E. (2007). User's manual for the SF-12v2 Health Survey (2nd ed.). Lincoln, RI: QualityMetric, Inc. 
Ware, J. E., Jr., Kosinski, M, \& Keller, S. D. (1996). A 12-item short-form health survey: Construction of scales and preliminary tests of reliability and validity. Medical Care, 34(3), 220-233.

Wehmeyer, M. L., \& Bolding, N. (2001). Enhanced self-determination of adults with intellectual disability as an outcome of moving to community-based work or living environments. Journal of Intellectual Disability Research, 45(5), 371-383.

Wehmeyer, M., \& Schwartz, M. (1998). The relationship between self-determination and quality of life for adults with mental retardation. Education and Training in Mental Retardation and Developmental Disabilities, 33, 3-12.

Welke, K. F., Morris, C. D., King, E., Komanapalli, C., Reller, M. D., \& Ungerleider, R. M. (2007). Population-based perspective of long-term outcomes after surgical repair of partial atrioventricular septal defect. The Annals of Thoracic Surgery, 84, 624-629. doi:10.1016/j.athoracsur.2007.03.079

Wells, M. (2010). Resilience in older adults living in rural, suburban, and urban areas. Online Journal of Rural Nursing and Health Care, 10(2), 45-54.

White-Scott, S, Spitalnik, D, Lunsky, Y., \& Havercamp, S. (2006), Women's health. In I. L. Rubin \& A. C. Crocker (Eds.) Medical Care for Children \& Adults with Developmental Disabilities (2nd ed. pp. 557-573). Baltimore, MD: Paul H. Brookes Publishing Co., Inc.

Wilson, I. B., \& Cleary, P. D. (1995). Linking clinical variables with health-related quality of life: A conceptual model of patient outcomes. JAMA: Journal of the American Medical Association, 273, 59-65.

Wilson, K. R., Jordan, J. A., \& Kras, A. M. (2010). Adolescent measures. In D. W. Nangle, D. J. Hansen, C. A. Erdley, \& P. J. Norton (Eds.), Practitioner's guide to empirically based measures of social skills (pp. 327-381). New York: Springer Science+Business Media, LLC.

Wiseman, F. K., Alford, K. A., Tybulewicz, V. L. J., \& Fisher, E. M. C. (2009). Down syndrome: Recent progress and future prospects. Human Molecular Genetics, 18(R1): R75-R83. doi: 10.1093/hmg/ddp010

Witts, P., \& Elders, S. (1998). The 'Severe Impairment Battery': Assessing cognitive ability in adults with Down syndrome. British Journal of Clinical Psychology, 37, 213-216.

Woolf, S., Woolf, C. M., \& Oakland, T. (2010). Adaptive behavior among adults with intellectual disabilities and its relationship to community independence.

Intellectual and Developmental Disabilities, 48(3), 209-215. doi:10.1352/19447558-48.3.209 
World Health Organization, Division of Mental Health and Prevention of Substance Abuse. (1997). Measuring quality of life. Retrieved from http://whqlibdoc.who.int/hq/1997/WHO_MSA_MNH_PSF 97.4.pdf

World Health Organization. (2003). Definition of Health. Retrieved from http://www.who.int/about/definition/en/print.html

Xavier, A.C., Ge, Y., \& Taub, J. (2010). Unique clinical and biological features of leukemia in Down syndrome children. Expert Review of Hematology, 3(2), 175 186. doi: 10.1586/ehm.10.14

Zigman, W. B. (2013). Atypical aging in Down syndrome. Developmental Disabilities Research Reviews, 18, 51-67. doi:10.1002/ddrr.1128

Zimmermann, F., \& Endermann, M. (2008). Self-proxy agreement and correlates of health-related quality of life in young adults with epilepsy and mild intellectual disabilities. Epilepsy \& Behavior, 13, 202-211. doi:10.1016/j.yebeh.2008.02.005 


\section{APPENDIX A. CONSENT FORM FOR PARTICIPANTS WITH DOWN SYNDROME}

\begin{tabular}{ll} 
Main Consent Form & $\begin{array}{l}\text { THEUNIVESITYOo } \\
\text { TENNESSEE }\end{array}$ \\
\hline
\end{tabular}

TITLE: The health-related quality of life of adults with Down syndrome

PRINCIPAL INVESTIGATOR: Rebecca Graves

112 Item Avenue

Mobile, Alabama

CO-INVESTIGATOR(S):

Carolyn Graff, $\mathrm{PhD}$

\section{INTRODUCTION:}

A person who takes part in a research study is called a research or study subject. In this consent form "you" refers to the research subject and/or the legally authorized representative.

You are being given the opportunity to participate in this research study. The purpose of this consent form is to help you decide if you want to be in the research study. This consent form may contain words that you do not understand. Please ask the researcher to explain any words or information that you do not clearly understand. We encourage you to talk with your family and friends before you decide to take part in this research study. Please tell the researcher if you are taking part in another research study.

The purpose of this study is to study the health-related quality of life of adults with Down syndrome.

About 60 pairs of people will be participating in this study from six different areas in the southeastern US, and about ten pairs of people in your local area will be participating.

The study will happen at places that are easy to get to and as private as possible, such as a private office, conference room, or examination room of a local medical clinic or a private group-study room or conference room of a local university library.

Your participation in this study will last no more than two hours.

\section{PROCEDURES TO BE FOLLOWED:}

Questionnaires, surveys and interviews:

- The researcher will ask your friend or family member to sit separately and answer questions given to him/her in a folder. These questions are about him/her and about you and your health.

- Your friend or family member will sit somewhere close, but where he/she cannot hear what you are saying so that you do not need to worry about him/her knowing what you are saying.

- The researcher will ask you questions about your health, and will audio-record what you are saying and take notes.

Page 1 of 7

February 21, 2013

Subject/Legally Authorized Representative Initials

TENNESTIS IRB NUMBER: 13-02403-XP

TENNESEEUT IRB APPROVAL DATE: 4/2/2013

IRB EXPIRATION DATE: 2/27/2014 
- Some of the questions may be personal or private, but the researcher will not share (or tell) your answers to anyone who knows you.

- Your name will not be used when the research is shared with other people.

- You will be talking and answering questions about your health in an interview with the researcher.

- You will answer questions on the Short Form-12v2 which will be about your health and how your health affects your life.

- The total time for the interview and survey will not be more than two hours.

- If you were not participating in this research study, none of these procedures would be done.

\section{RISKS ASSOCIATED WITH PARTICIPATION:}

You are not in any kind of danger talking to the researcher and answering questions. Some questions may be embarrassing, but they are important questions to learn about how to understand your health. Your answers will not be told to anyone who knows you. When what you say is used in the study, no one will use your name. The research may involve risks to you which are currently unforeseeable. You will be told about any new information that might change your decision to be in this study. You may be asked to sign a new consent form if this occurs.

There is the potential risk of loss of confidentiality. Every effort will be made to keep your information confidential; however, this cannot be guaranteed.

\section{Questionnaires/Surveys:}

Completion of the Short Form-12v2 Health survey may make you feel uncomfortable or cause troublesome feelings or emotions. You may refuse to answer any of the questions and you may take a break at any time during the study.

\section{Audio Recording:}

Having your voice recorded may make you feel uncomfortable. You may take a break during any time of the study. There is also a potential risk of loss of confidentiality that someone who hears your audio recording might identify you.

\section{BENEFITS ASSOCIATED WITH PARTICIPATION:}

You will not receive any benefits from being in this study, but the answers you give can help nurses and other health-care workers understand the health of adults with Down syndrome.

\section{ALTERNATIVES TO PARTICIPATION:}

You do not have to participate in this study.

Page 2 of 7

February 21, 2013

Subject/Legally Authorized Representative Initials 
If you choose not to participate in this research study, you will not have to answer questions about your health and your friend or family member will not answer any questions about you.

\section{CONFIDENTIALITY:}

\section{Research records}

All your paper research records will be stored in locked file cabinets and only the researchers will be able to look at them.

All your electronic research records will be computer password protected and only the researchers will be able to look at them.

Information about your participation in this study or the results of procedures performed in this study will not be placed in your medical record; as such, this information will not be made available to your employer or insurer.

\section{Presentations/Publications}

Your name will not be used in any presentations or publications based on the results of this research study.

\section{Authorization to Use and Disclose Information for Research Purposes}

Under federal privacy regulations, you have the right to decide who can review and copy your personal health information (called "protected health information" or PHI). PHI collected in this study may include information such as:

- Records about your study visits

- Records about phone calls made as part of this research

- Research records

By signing this consent form, you are giving your permission for the researcher and the study staff at the University of Tennessee to share your PHI with the Institutional Review Board (IRB) at the University of Tennessee Health Science Center.

Your PHI will only be used and/or given to others:

- to do the research,

- to study the results, and

- to see if the research was done right.

Your PHI will be used until the study is completed.

You may withdraw or take away your permission to use and disclose your PHI at any time. You do this by sending written notice to the researcher. If you withdraw your permission, you may not be able to stay in the study.

Page 3 of 7

February 21, 2013

Subject/Legally Authorized Representative Initials 
When you withdraw your permission, no new PHI will be gathered after that date. However, information that has already been gathered may still be used and given to others. The federal regulations allow you to review or copy your PHI that is used in this study.

\section{COMPENSATION AND TREATMENT FOR INJURY:}

You are not waiving any legal rights or releasing the University of Tennessee or its agents from liability for negligence. In the event of physical injury resulting from research procedures, the University of Tennessee does not have funds budgeted for compensation for medical treatment. Therefore, the University of Tennessee does not provide for treatment or reimbursement for such injuries.

If you are injured or get sick as a result of being in this study, call the study researcher immediately. The study researcher will provide you with a subsequent referral to appropriate health care facilities.

If you are injured or get sick as a result of being in this study, you and/or your insurance will be billed for the costs associated with this medical treatment.

No compensation will be available to you for any extra expenses that you may have as the result of research related physical injuries, such as additional hospital bills, lost wages, travel expenses, etc.

No compensation will be available to you for any non-physical injuries that you may have as a result of research participation, such as legal problems, problems with your finances or job, or damage to your reputation.

\section{QUESTIONS:}

Contact Rebecca Graves at 251-490-1528 if you have questions about your participation in this study or if you have questions, concerns, or complaints about the research.

If you feel you have had a research-related injury, contact Rebecca Graves at 251-490-1528 any time of the day and any day of the week. If there is no answer, please leave a message and your call will be returned.

You may contact Terrence F. Ackerman, Ph.D., UTHSC IRB Chairman, at 901-448-4824 or visit the IRB website at

http://www.uthsc.edu/research/research compliance/IRB/participant complaint.php if you have any questions about your rights as a research subject or if you have questions, concerns, or complaints about the research.

Page 4 of 7

February 21, 2013

Subject/Legally Authorized Representative Initials

TENNESTYA IRB NUMBER: 13-02403-XP

TENNESSEEUT

IRB EXPIRATION DATE: 2/27/2014 


\section{PAYMENT FOR PARTICIPATION:}

You will receive a \$10 gift card to Wal-Mart or to Target at the end of the study visit on the day of the visit if you decide to complete the study.

\section{COSTS OF PARTICIPATION:}

There are no costs to you for participating in this study.

\section{VOLUNTARY PARTICIPATION AND WITHDRAWAL:}

Your participation in this research study is voluntary. You may decide not to participate or you may leave the study at any time. Your decision will not result in any penalty or loss of benefits to which you are entitled.

If you decide to stop being part of the study, you should tell your researcher, and any information that you have already provided will be kept in a confidential manner.

Your participation in this research study may be stopped by the researcher without your consent for any of the following reasons:

- If you do not answer the researcher's questions; or

- If you do not talk about your health

\section{PERMISSION FOR CAREGIVER TO ANSWER QUESTIONS ABOUT YOU:}

What is the name of the friend or family member who is allowed to answer questions about you and your health?

Page 5 of 7

February 21, 2013

Subject/Legally Authorized Representative Initials 


\section{CONSENT OF SUBJECT:}

You have read or have had read to you a description of the research study as outlined above. The investigator or his/her representative has explained the study to you and has answered all the questions you have at this time. You knowingly and freely choose to participate in the study. A copy of this consent form will be given to you for your records.

Signature of Research Subject (18 years + )

Printed Name of Adult Research Subject

Signature of Legally Authorized Representative

Printed Name of Legally Authorized Representative

Relationship of Legally Authorized Representative

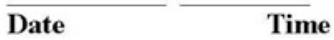

Signature of Person Obtaining Consent

$\overline{\text { Date }}$ Time

\section{Printed Name of Person Obtaining Consent}

In my judgment, the subject or the legally authorized representative has voluntarily and knowingly given informed consent and possesses the legal capacity to give informed consent to participate in this research study.

Page 6 of 7

February 21, 2013 


\section{Assent Obtained}

The assent discussion was initiated on

(date) at

(time).

The information was presented in developmentally-appropriate terms.

Subject's Printed Name

$\overline{\text { Subject's Signature }} \overline{\text { Date }} \overline{\text { Time }}$

* Please note that the legally authorized representative(s) must sign the consent signature page.

I hereby certify that I have discussed the research project with the subject and/or his/her legally authorized representative(s). I have explained all the information contained in the informed consent document, including any risks that may be reasonably expected to occur. I further certify that the research subject was encouraged to ask questions and that all questions were answered.

Printed Name of Person Obtaining Assent

Signature of Person Obtaining Assent

Date

Time

Page 7 of 7

February 21, 2013

Subject/Legally Authorized Representative Initials

TENNESSTY IRB NUMBER: 13-02403-XP

TEN NESSEEUT RBR APPROVAL DATE: 4/2/2013

IRB EXPIRATION DATE: 2/27/2014 


\section{APPENDIX B. CONSENT FORM FOR CAREGIVER PARTICIPANTS}

\begin{tabular}{ll} 
Caregiver Consent Form & THENIVERSITYof \\
TENNESSEE \\
HEALTH SCIENCE CENTER \\
\hline
\end{tabular}

TITLE: The health-related quality of life of adults with Down syndrome

$\begin{array}{ll}\text { PRINCIPAL INVESTIGATOR: } & \text { Rebecca Graves } \\ & 112 \text { Item Avenue } \\ & \text { Mobile, Alabama } \\ \text { CO-INVESTIGATOR(S): } & \text { Carolyn Graff, PhD }\end{array}$

1. INTRODUCTION:

You are being given the opportunity to participate in this research study. The purpose of this consent form is to help you decide if you want to be in the research study. This consent form may contain words that you do not understand. Please ask the researcher to explain any words or information that you do not clearly understand. We encourage you to talk with your family and friends before you decide to take part in this research study. Please tell the researcher if you are taking part in another research study.

The purpose of this study is to find out what you think about the health of your friend, family member, or patient with Down syndrome and how his/her health affects his/her life.

About 60 pairs of people will be participating in this study from six different areas in the southeastern US, and about ten pairs of people in your local area will be participating.

The study will happen at places that are easy to get to and as private as possible, such as a private office, conference room, or examination room of a local medical clinic or a private group-study room or conference room of a local university library.

Your participation in this study will last no more than two hours.

\section{PROCEDURES TO BE FOLLOWED:}

Questionnaires and surveys:

The researcher will ask you to sit separately and answer questions on four different forms given to you in a folder.

- Demographic Form has questions about you, the adult with Down syndrome, and the relationship that the two of you have.

- SF-12v2 Health Survey has questions about the general health and activity of the adult with Down syndrome.

- Health Questionnaire has questions about specific health problems, health risks, and health-care utilization of the adult with Down syndrome.

- Vineland-II has questions about abilities and behaviors of the adult with Down syndrome. 
- It will take between one to one-and-a-half hours to complete the questionnaires and surveys.

- If you were not participating in this research study, none of these procedures would be done.

\section{RISKS ASSOCIATED WITH PARTICIPATION:}

You are not in any kind of danger taking part in this study. Some questions may be embarrassing, but they are important questions to learn about how to understand the health of adults with Down syndrome. Your answers will not be told to anyone who knows you. When what you say is used in the study, no one will use your name.

There is the potential risk of loss of confidentiality. Every effort will be made to keep your information confidential; however, this cannot be guaranteed.

The research may involve risks to you which are currently unforeseeable. You will be told about any new information that might change your decision to be in this study. You may be asked to sign a new consent form if this occurs.

\section{Questionnaires/Surveys:}

Completion of the questionnaires and surveys may make you feel uncomfortable or cause troublesome feelings or emotions. You may refuse to answer any of the questions and you may take a break at any time during the study.

\section{Audio Recording:}

Having your voice recorded may make you feel uncomfortable. You may take a break during any time of the study. There is also a potential risk of loss of confidentiality that someone who hears your audio recording might identify you.

\section{BENEFITS ASSOCIATED WITH PARTICIPATION:}

You will not receive any benefits from being in this study, but the answers you give can help nurses and other health-care workers understand the health of adults with Down syndrome better.

\section{ALTERNATIVES TO PARTICIPATION:}

You do not have to participate in this study.

You will not have to undergo the following procedures if you do not take part in this study:

- Demographic Form

- SF-12v2 Health Survey

- Health Questionnaire 
- Vineland-II Survey

\section{CONFIDENTIALITY:}

Research records

All your paper research records will be stored in locked file cabinets and only the researchers will be able to look at them.

All your electronic research records will be computer password protected and only the researchers will be able to look at them.

\section{Presentations/Publications}

Your name will not be used in any presentations or publications based on the results of this research study.

\section{COMPENSATION AND TREATMENT FOR INJURY:}

You are not waiving any legal rights or releasing the University of Tennessee or its agents from liability for negligence. In the event of physical injury resulting from research procedures, the University of Tennessee does not have funds budgeted for compensation for medical treatment. Therefore, the University of Tennessee does not provide for treatment or reimbursement for such injuries.

If you are injured or get sick as a result of being in this study, call the study researcher immediately. The study researcher will provide you with a subsequent referral to appropriate health care facilities.

If you are injured or get sick as a result of being in this study, you and/or your insurance will be billed for the costs associated with this medical treatment.

No compensation will be available to you for any extra expenses that you may have as the result of research related physical injuries, such as additional hospital bills, lost wages, travel expenses, etc.

No compensation will be available to you for any non-physical injuries that you may have as a result of research participation, such as legal problems, problems with your finances or job, or damage to your reputation.

\section{QUESTIONS:}

Contact Rebecca Graves at 251-490-1528 if you have questions about your participation in this study or if you have questions, concerns, or complaints about the research. 
If you feel you have had a research-related injury, contact Rebecca Graves at 251-490-1528 any time of the day and any day of the week. If there is no answer, please leave a message and your call will be returned.

You may contact Terrence F. Ackerman, Ph.D., UTHSC IRB Chairman, at 901-448-4824 or visit the IRB website at

http://www.uthsc.edu/research/research compliance/IRB/participant complaint.php if you have any questions about your rights as a research subject or if you have questions, concerns, or complaints about the research.

\section{PAYMENT FOR PARTICIPATION:}

You will receive a $\$ 10$ gift card to Wal-Mart or to Target at the end of the study visit on the day of the visit if you decide to complete the study.

\section{COSTS OF PARTICIPATION:}

There are no costs to you for participating in this study.

\section{VOLUNTARY PARTICIPATION AND WITHDRAWAL:}

Your participation in this research study is voluntary. You may decide not to participate or you may leave the study at any time. Your decision will not result in any penalty or loss of benefits to which you are entitled.

If you decide to stop being part of the study, you should tell your researcher, and any information that you have already provided will be kept in a confidential manner.

Your participation in this research study may be stopped by the researcher without your consent for any of the following reasons:

- If you do not answer the surveys and/or questionnaires.

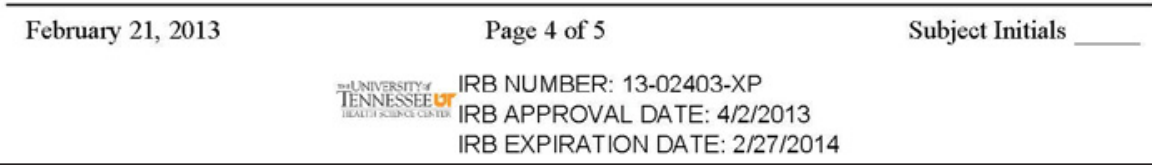




\section{Caregiver Consent Form}

\section{CONSENT OF SUBJECT:}

You have read or have had read to you a description of the research study as outlined above. The investigator or his/her representative has explained the study to you and has answered all the questions you have at this time. You knowingly and freely choose to participate in the study. A copy of this consent form will be given to you for your records.

Signature of Research Subject

Printed Name of Research Subject

Signature of Person Obtaining Consent $\overline{\text { Date }} \overline{\text { Time }}$

Time

\section{Printed Name of Person Obtaining Consent}

In my judgment, the subject or the legally authorized representative has voluntarily and knowingly given informed consent and possesses the legal capacity to give informed consent to participate in this research study. 


\section{Adults with Down syndrome and a parent, close relative, or friend}

If you are an adult with Down syndrome and at least 18 years old, or a close relative or friend, your help is needed in a research study.

You may help in this research study to learn about the health of adults with Down syndrome.

Both the adult with Down syndrome and the relative or friend will receive a $\$ 10$ gift card for answering questions. You will receive a gift card from Wal-Mart or Target.

We will meet at a local medical clinic or local library.

The meeting takes between $11 / 2$ to 2 hours.

\section{Call or email Rebecca Graves}

University of Tennessee Health Science Center

Student

RGraves@southalabama.edu

251-490-1528 


\section{APPENDIX D. DEMOGRAPHIC FORM}

\section{Demographic Form}

Please answer the following questions about yourself (companion/caregiver)

1. What is your age?

2. What is your gender? (Please check one)

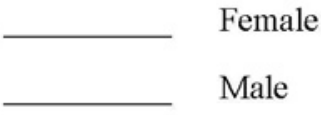

3. What is your ethnicity or race? (Please check all that apply).

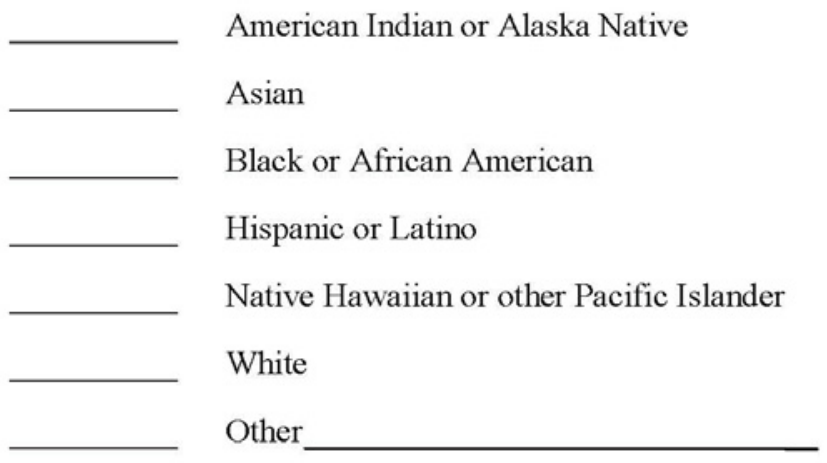

4. What is your marital status? (Please check one)

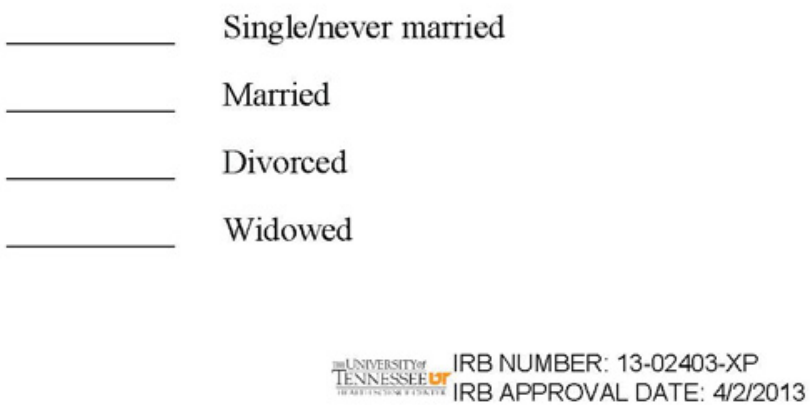


5. What is the highest level of education you have completed? (Please check one)

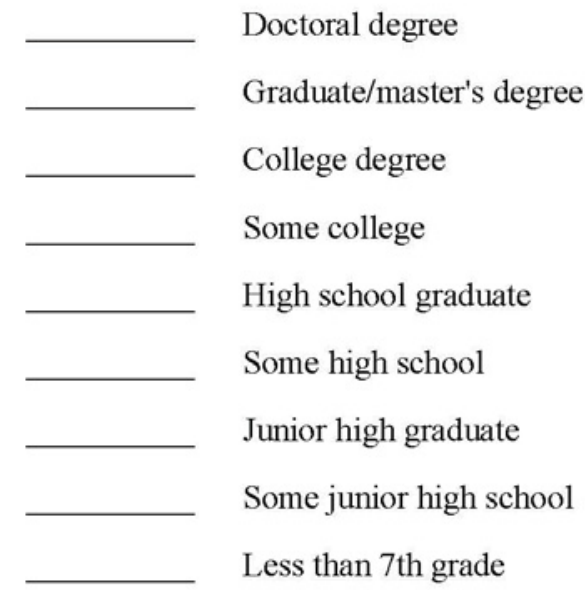

6. What is your current employment status? (Please check one)

\section{Retired}

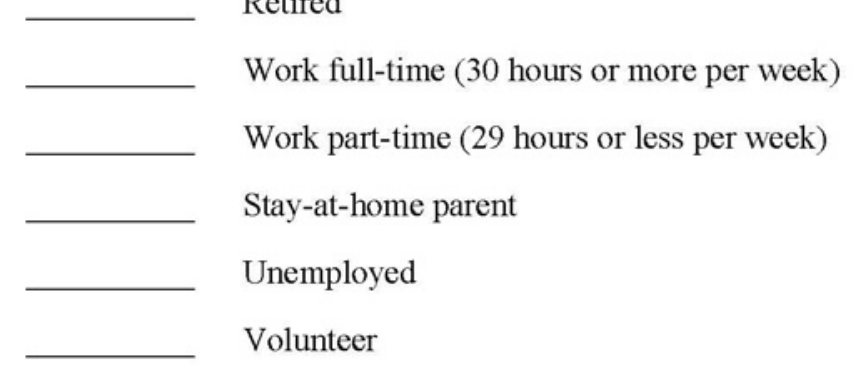

7. What is your relationship to adult with Down syndrome? (Please check one)

Parent

Sibling

Other family relationship (please identify) 


\section{Friend}

Significant other

8. How often do you see the adult with Down syndrome? (please check one)

Daily

Five to six times per week

Three to four times per week

Once or twice per week

Three to four times per month

Once or twice per month

Nine to eleven times per year

Six to eight times per year

Three to five times per year

Once or twice per year

9. How often do you talk to the adult with Down syndrome by telephone or have a conversation with him or her by text or email? (please check one)

Daily

Five to six times per week

Three to four times per week

Once or twice per week 
Three to four times per month

Once or twice per month

Nine to eleven times per year

Six to eight times per year

Three to five times per year

Once or twice per year

Please answer the following questions about the adult with Down syndrome:

1. What is his/her age?

2. What is his/her gender? (Please check one)

Female

Male

3. What is his/her ethnicity or race? (Please check all that apply).

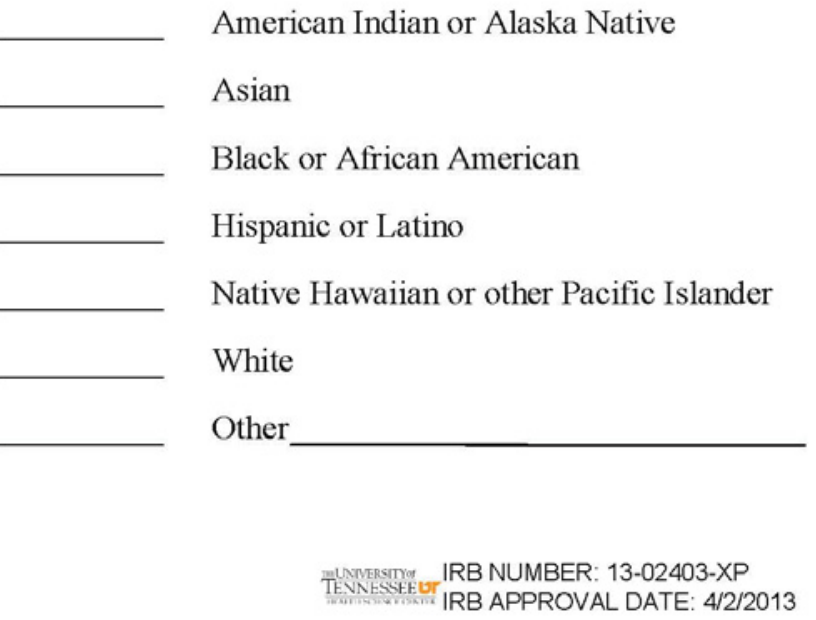


4. What is his/her marital status? (Please check one)

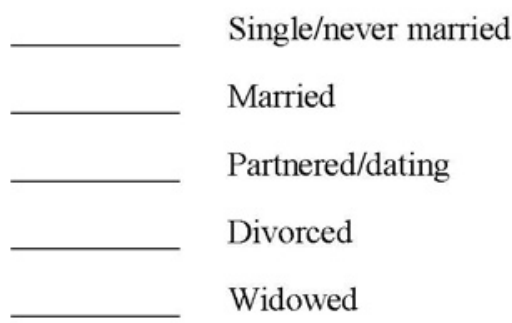

5. What is the highest level of education he/she has completed? (Please check one)

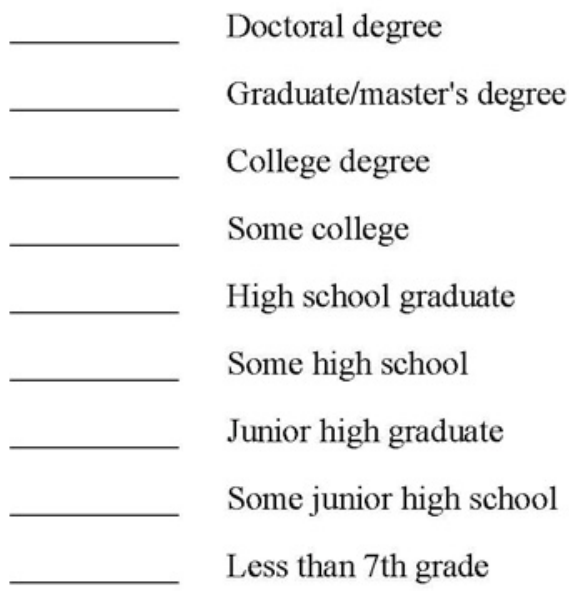

6. What is his/her current employment status? (Please check one)

Retired

Work full-time (30 hours or more per week)

Work part-time (29 hours or less per week)

PULNERSTrom IRB NUMBER: 13-02403-XP

TEVNESSEEUT IRB APPROVAL DATE: 4/2/2013 


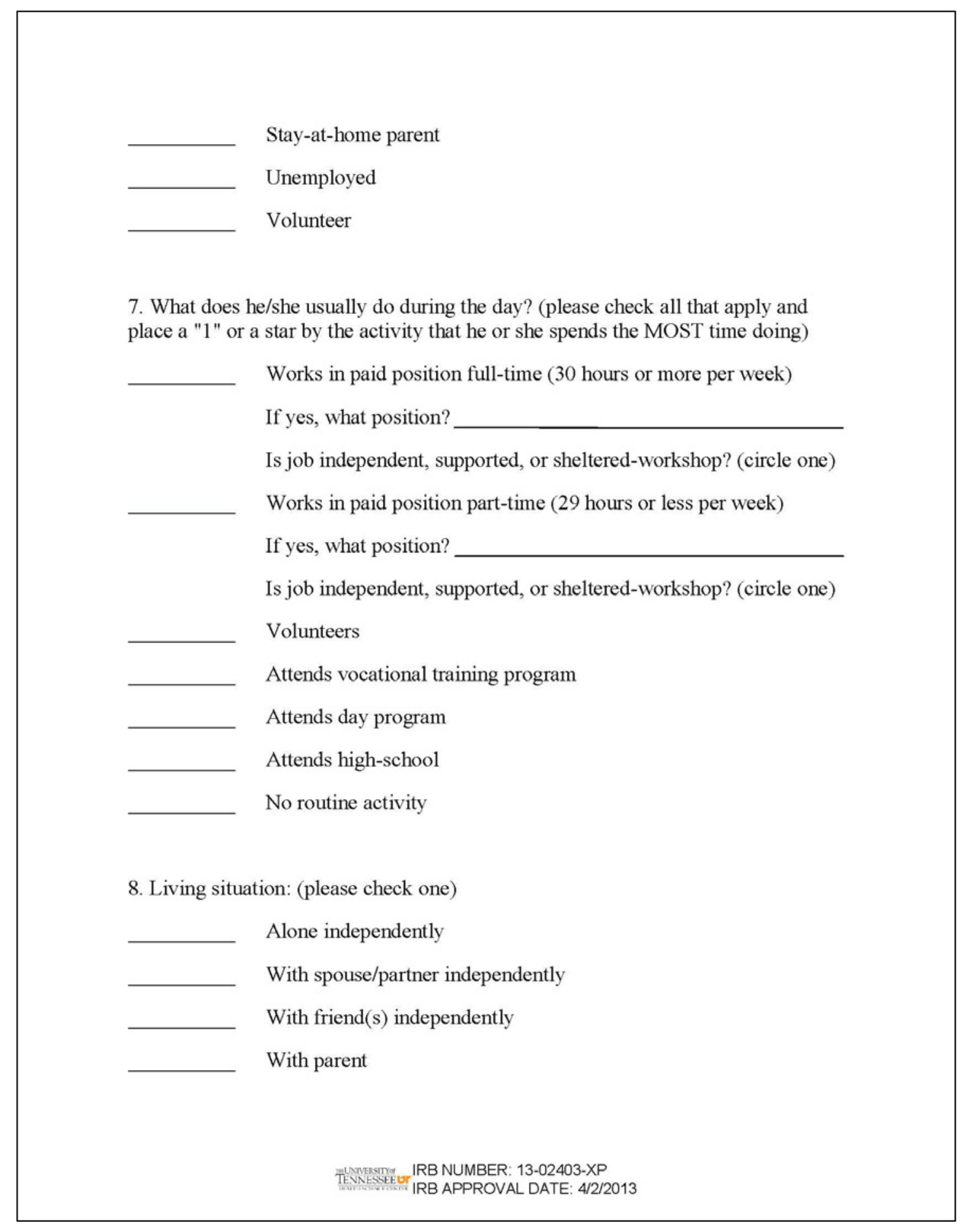


With sibling

With other relative (relationship)

In a group home

In a supervised or staffed environment (please describe)

Other

9. Does the adult with Down syndrome live with you? (yes/no) 
ID Number

\section{Health Questionnaire}

You are answering these questions about the health of the adult with Down syndrome. If you would like to give more information about a question, please feel free to write your comments next to the answer. If you need more room, feel free to turn the page over and write on the back. If you write on the back of a page, please include the number of the question you are commenting on. Please do not discuss this with the adult with Down syndrome, as he/she will be answering many of the same questions. Thank you.

\section{Health Problems}

Does he/she have:

\section{Musculoskeletal}

1. Trouble walking?

Yes__ No

2. Trouble getting out of a chair?

Yes__ No

3. Aches and pains?

Yes___ No___ If yes, where?

4. Arthritis?

Yes___ No___ If yes, where?

Neurosensory

5. Seizures?

Yes

No 
ID Number:

6. Dizziness or weak spells?

Yes

No

7. Headaches?

Yes

No

8. Problems hearing?

Yes

No

9. Problems seeing?

Yes

No

10. Problems reading or identifying small objects?

Yes

No

11. Feeling sad or blue?

Yes

No

12. Problems remembering?

Yes

No

\section{Endocrine}

13. Problems with his/her appetite?

Yes

No

14. Problems with his/her weight?

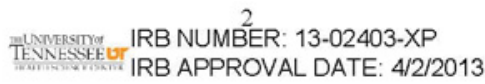


ID Number:

Yes

No

15. Thyroid problems?

Yes

No

16. Diabetes?

Yes

No

\section{Gastrointestinal/Genitourinary}

17. To go to the bathroom at night?

Yes

No

If yes, how many times per night?

18. Problems with his/her teeth or gums?

Yes

No

19. Heartburn?

Yes

No

20. Problems with constipation?

Yes

No

21a. Problems with monthly period or menstrual cycle (irregular, heavy, or painful cramps)? (women only)

Yes

No

If her period stopped (menopause), when?

21b. Prostate problems? (men only)

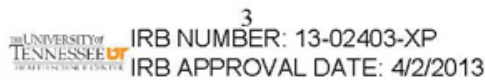


ID Number:

Yes

No

Cardiovascular

22. High blood pressure?

Yes

No

23. Heart problems now or in the past?

Yes__ No___ If yes, what type?

24. Circulatory problems/blood clots?

Yes___ No___ If yes, what type?

\section{Respiratory}

25. Problems with breathing while asleep?

Yes

No

26. Colds often?

Yes

No

27. Problems with coughing?

Yes

No

28. Shortness of breath?

Yes

No

29. Asthma?

Yes

No 
ID Number:

Immune

30. Cancer?

Yes__ No___ If yes, what type?

31. Allergies?

Yes__ No___ If yes, what type?

32. Does he/she have any other health problems we have not talked about?

Yes__ No___ If yes, what are they?

\section{Health Risks}

33. Does he/she smoke?

Yes ___ No___ If yes, how many packs a day?

Is he/she often around others who smoke?

34. Does he/she ever drink beer, wine, or alcohol?

Yes

No

If yes, what does he/she drink?

How much/How often?

35. Does he/she use any street drugs?

Yes

No

If yes, what street drugs?

How much/How often?

36. Is he/she sexually active?

Yes

No

How many partners does he/she have?

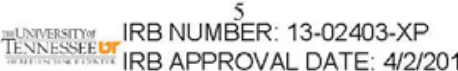


ID Number:

If yes, does he/she use birth control? What type?

\section{Health-Care Utilization}

Please make a check mark in the box for the last time he/she saw:

\begin{tabular}{|l|l|l|l|l|}
\hline & $\begin{array}{c}\text { In the last } \\
\text { month? }\end{array}$ & $\begin{array}{c}\text { In the last } \\
1 \text { year? }\end{array}$ & $\begin{array}{c}\text { In the last } \\
\text { 2 years? }\end{array}$ & $\begin{array}{c}\text { Other } \\
\text { (please } \\
\text { indicate) }\end{array}$ \\
\hline $\begin{array}{l}\text { 37. A health care provider } \\
\text { (doctor, nurse practitioner or } \\
\text { physician assistant)? }\end{array}$ & & & & \\
\hline 38. A dentist? & & & & \\
\hline 39. An eye specialist? & & & & \\
\hline $\begin{array}{l}\text { 40. An emergency department or } \\
\text { urgent care center? }\end{array}$ & & & & \\
\hline
\end{tabular}

Please make a check mark in the box for the last time he/she had:

\begin{tabular}{|c|c|c|c|c|}
\hline & $\begin{array}{l}\text { In the last } \\
\text { month? }\end{array}$ & $\begin{array}{l}\text { In the last } \\
\text { year? }\end{array}$ & $\begin{array}{l}\text { In the last } \\
2 \text { years? }\end{array}$ & $\begin{array}{c}\text { Other } \\
\text { (please } \\
\text { indicate) }\end{array}$ \\
\hline 41. His/her hearing checked? & & & & \\
\hline $\begin{array}{l}\text { 42a. An exam of the testicles } \\
\text { (men only)? }\end{array}$ & & & & \\
\hline $\begin{array}{l}\text { 42b. A female (internal or pap) } \\
\text { exam (women only)? }\end{array}$ & & & & \\
\hline $\begin{array}{l}\text { 43a. A prostate exam (men } \\
\text { only)? }\end{array}$ & & & & \\
\hline $\begin{array}{l}\text { 43b. A mammogram or breast } \\
\text { ultrasound (women only)?? }\end{array}$ & & & & \\
\hline 44. His/her thyroid checked & & & & \\
\hline
\end{tabular}


ID Number:

\begin{tabular}{|l|l|l|l|l|}
\hline 45. A neck x-ray & & & & \\
\hline 46. A TB test? & & & & \\
\hline 47. A flu shot? & & & & \\
\hline
\end{tabular}

48. Does he/she have a regular primary care provider (doctor, nurse practitioner, or physician assistant)?

Yes

No

49. What type of health insurance does he/she have?

Private Medicaid Medicare None Other

50. Does he/she feel unable to get to a health care provider?

Yes

No

If yes, check the reason why:

Does not know who to go to
Does not know where to go
Transportation problems
Work problems
Appointments take too much time
$\quad$ Does not like or trust the health care provider
$\square \quad$ Feels he/she is not understood or respected
Is afraid of the health care provider
$\quad$ Clinics are too far away, inconvenient
$\quad$ Does not have enough help from others
$\quad$ Does not have enough support from others

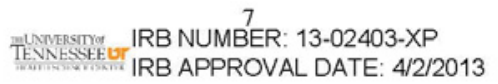


ID Number:

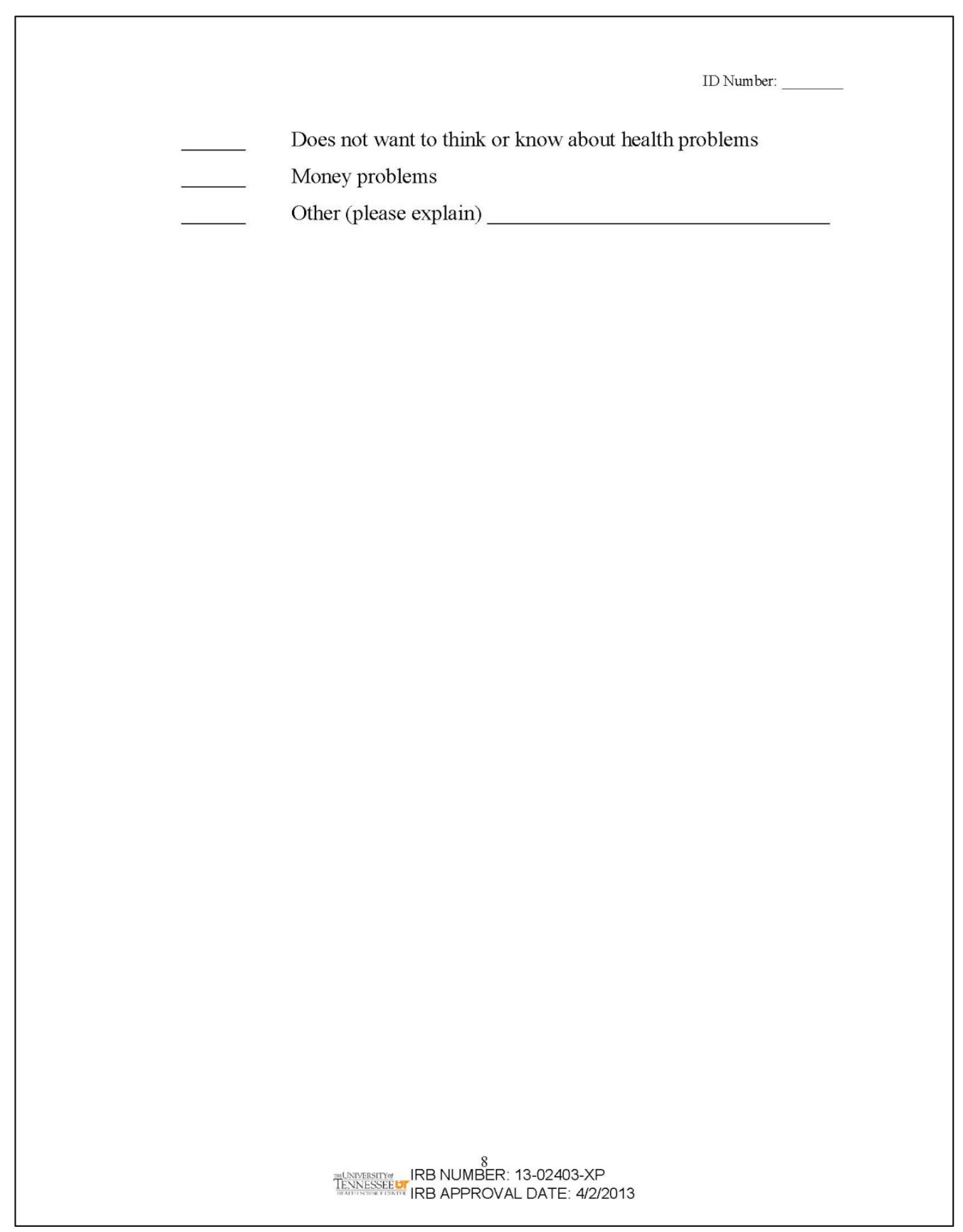

Source. Adapted with permission from a poster session. Graff, J. C., Engle, V. F., \& Pruett, J. (2003, November). Health disparities of custodial and non-custodial black grandparents caring for grandchildren. Poster session presented at the $56^{\text {th }}$ annual meeting of the Gerontological Society of America, San Diego, CA. 
ID Number:

\section{GUIDE FOR INTERVIEWING ADULTS WITH DOWN SYNDROME ABOUT THEIR HEALTH PROBLEMS, RISKS, AND UTILIZATION}

I am going to ask you questions about your health. I will ask you about times you feel good. I will ask you about when you feel bad or sick. Let's begin.

\section{Health Problems}

1. Tell me about health.

Probe: What does health mean to you? What do you think when I say 'health'? What does 'being sick' mean to you?

2. Tell me about your health.

Probe: Do you think you are healthy?

Probe: Do you think your body is healthy?

If YES: Tell me what you do to stay healthy.

If NO: You do not feel healthy. Tell me about it.

3. Tell me how you feel most of the time.

Probe: Do you feel good most of the time?

Probe: Does your body feel good most of the time?

If YES: What helps you to feel good?

If NO: You feel bad some of the time. Tell me about those times. Are there times that you do feel good? Tell me about those times that you do feel good.

4. Tell me about any problems you have when you don't feel good.

Expand on the answer: When (response), how do you feel?

What about (response) makes you not feel good?

When (response), what do you do?

When (response), what do you think?

When (response), what do other people do?

When (response), what do other people do to help you?

Try to elicit the root activity or behavior that might indicate or contribute to a health problem without leading (e.g., pain, headache, sleep problems, problems walking, feeling sad or blue etc.) 
ID Number:

5. Are there times you feel sick? Tell me about those times.

If NO: So you do not get sick, is that right? How do you keep from getting sick?

If not understanding: Do you sometimes not feel good? Tell me about that

Try to elicit the root activity or behavior that might indicate or contribute to a health problem without leading (e.g., pain, headache, sleep problems, problems walking, feeling sad or blue etc.)

6. Are there times something hurts? Tell me about those times.

Expand on the answer: When (response), what do you do?

When (response), what do you think?

When (response), what do other people do?

When (response), what do other people do to help you?

\section{Health Risks}

7. Are there things that you do that could make you sick? Tell me about those things.

If NO: Are there things that you do to keep from getting sick? Tell me about those things.

8. Tell me about things you did when you were a child that made you sick when you got older.

Probe: What do you think about smoking cigarettes?

What do you think about drinking beer or wine?

9. What can help you stay healthy?

Probe: What can help you keep from being sick?

Who can help you the most?

\section{Health Utilization}

10. People go to the doctor when they get sick. People go to the doctor to stay healthy or well. Tell me about when you go to the doctor. 
ID Number:

11. When was the last time that you went to the doctor? Tell me about that.

12. What does the doctor do when you go?

13. What do you think doctors could do to help you?

14. What do you think nurses could do to help you?

15. Can you tell me about any problems that you have going to the doctor?

Probe: Do you think it is hard to go to the doctor? Why or tell me about that? Do you think it is easy to go to the doctor? Why or tell me about that? Is there something you really like about going to the doctor? What or tell me about that?

Is there something you really do not like about going to the doctor? What or tell me about that?

16. How many different doctors do you see? Tell me what each doctor does for you.

Probe: Do you have one doctor? Or, do you have two or three doctors you go to see? Do you go to a doctor who checks your eyes? Tell me about that. Do you go to a doctor who checks your teeth? Tell me about that. Do you go to a doctor who checks your hearing? When?

\section{Summary and Closing}

17. Thank you for talking with me today. You helped me learn about your health. You helped me learn what you do to stay healthy. Is there anything else you want to tell me?

18. You talked about (interviewer will make one or two statements that summarize major points made by the adult with Down syndrome). Is this correct? Is there anything you want to add?

19. Would you like to ask me a question? 


\section{VITA}

Rebecca Jermyn Graves, born in 1971 in Gulfport, Mississippi, earned the Doctor of Philosophy from the University of Tennessee Health Science Center in May, 2014. Her dissertation research, for which she was awarded funding by the Beta Theta-at-large chapter of Sigma Theta Tau International Honor Society of Nursing and from the Down Syndrome Society of Mobile County, investigated the health-related quality of life of adults with Down syndrome.

While a doctoral student at the University of Tennessee Health Science Center, Rebecca was selected for a funded Leadership Education in Neurodevelopmental and Related Disabilities Traineeship for two consecutive years at the Boling Center for Developmental Disabilities in Memphis, Tennessee. Through this traineeship, Rebecca co-authored one peer-reviewed publication and conducted and contributed to multiple professional presentations. She was lead author on two poster presentations that described the secondary analysis of data from a national survey of siblings of adults with Down syndrome. She collected, entered, and analyzed data from several studies conducted by researchers with the Boling Center for Developmental Disabilities.

Rebecca earned a Bachelor of Science in Nursing in 2001, a Master of Science in Nursing in 2006, and a Post-Master degree in 2009 from the University of South Alabama. She is a certified family nurse practitioner and an assistant professor of adult health nursing at the University of South Alabama. She was a co-investigator for a grantfunded research study on the use of simulation in the initial clinical orientation for nursing students, resulting in a publication in a peer-reviewed journal, which she coauthored. She also earned a Bachelor of Science in journalism, emphasis public relations from the University of Southern Mississippi in 1996. 